\title{
Homomorfismos Contínuos entre \\ Álgebras de Germes Holomorfos
}

\author{
Luciano O. Condori Huanca
}

Tese Apresentada ao

INSTITUTO DE MATEMÁTICA E ESTATÍSTICA

da

UNIVERSIDADE DE SÃO PAULO

para a obtenção do grau de doutor em matemática

Área de concentração: Análise

Orientadora: Prof $\stackrel{\text { a }}{\text {. Dr a }}$. Mary Lilian Lourenço

Durante a elaboração deste trabalho, o autor recebeu apoio financeiro da CNPQ

São Paulo, julho de 2001. 


\title{
Homomorfismos Contínuos entre Álgebras de Germes Holomorfos
}

\author{
Este exemplar corresponde à redação final \\ da dissertação devidamente corrigida e \\ defendida por Luciano O. Condori Huanca \\ e aprovada pela comissão julgadora.
}

São Paulo, outubro de 2001.

Banca examinadora:

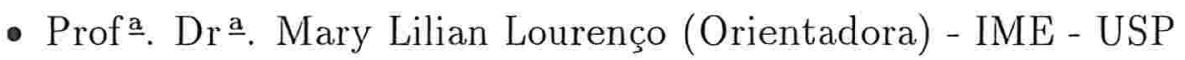

- Prof. Dr. Jorge Aragona - IME - USP

- Prof. Dr. Jorge Mujica - IMECC - UNICAMP

- Prof. Dr. Mario C. Matos - IMECC - UNICAMP

- Prof $\stackrel{a}{\text {. Dr }}$. Luiza A. Moraes - IM - UFRJ 
A minha esposa:

Maria das Graças

e aos meus filhos:

Luana, Rebeca e Joaquin Lorenzo. 


\title{
Resumo
}

Este trabalho tem por objetivo estudar homomorfismos contínuos entre álgebras de germes holomorfos em um compacto de um espaço de Banach, quando munida da topologia de Nachbin. Apresentamos condições necessárias e suficientes para que tais homomorfismos sejam operadores de composição. A partir daí estudamos condições necessárias e suficientes para que tais operadores de composição sejam fracamente compactos e compactos. No último capítulo passamos a estudar homomorfismos contínuos entre álgebras de germes de funções holomorfas em um compacto a valores em uma álgebra de Banach e apresentamos resultados para que os mesmos sejam operadores de composição, fracamente compactos e compactos.

\begin{abstract}
The main purpose of this work is to study $\tau_{w}$-continuous homomorphisms between algebras of holomorphic germs on a compact subset of a Banach space. We present equivalent conditions in such a way that these homomorphisms are composition operators. We also present equivalent conditions for the above composition operator to be compact and weakly compact. Finally we study $\tau_{w}$-continuous homomorphisms between algebras of vector-valued holomorphic germs on a compact subset of a Banach space and we also present results such that the $\tau_{w}$-continuous homomorphisms are composition operators, weakly compact and compact.
\end{abstract}




\section{Agradecimentos}

- Ao professor Jorge Mujica da Universidade Estadual de Campínas, pelas valiosas sugestões e por ter sanado algumas dúvidas que surgiram ao longo desta tese.

- E principalmente à professora Mary Lilian Lourenço pela sugestão do tema, pela paciente e valiosa orientação, sem a qual eu não teria concluído esta tese.

- Ao CNPQ pelo apoio financeiro no decorrer desta tese.

- A todas as pessoas que de alguma maneira nos ajudaram a concluir este trabalho.

- A minha esposa Maria das Graças e aos meus filhos Luana, Rebeca e Joaquin Lorenzo.

* Obrigado * 


\section{Índice}

Introdução 1

1 Germes de funções holomorfas $\quad 5$

1.1 Notações . . . . . . . . . . . . . . . . . . . . 5

1.2 Definições e resultados preliminares sobre germes holomorfos . . . . . . . 7

1.3 Algumas propriedades de limites indutivos . . . . . . . . . . . . . 11

$\begin{array}{lll}2 & \text { Operadores de composição } & 17\end{array}$

2.1 Homomorfismos contínuos entre álgebras de germes holomorfos em espaços de dimensão finita . . . . . . . . . . . . . . . . . . . . . . 17

2.2 Homomorfismos contínuos entre certas álgebras de funções holomorfas . . . 24

$3 \quad$ Homomorfismos contínuos entre álgebras de germes holomorfos 31

3.1 Homomorfismos contínuos entre álgebras de germes holomorfos . . . . . . . 31

3.2 Homomorfismos contínuos entre as álgebras $H(U)$ e $\mathcal{H}(K) \quad \ldots \ldots$. . . . 37

3.3 Homomorfismos compactos entre álgebras de germes holomorfos . . . . . . 39

3.4 Operadores de composição fracamente compactos entre espaços de germes . 42

4 Homomorfismos contínuos entre álgebras de germes holomorfos a valo- 
4.1 Operadores de composição entre álgebras de funções holomorfas a valores vetoriais . . . . . . . . . . . . . . . . . . . . . 47

4.2 Operadores de composição entre álgebras de germes holomorfos a valores

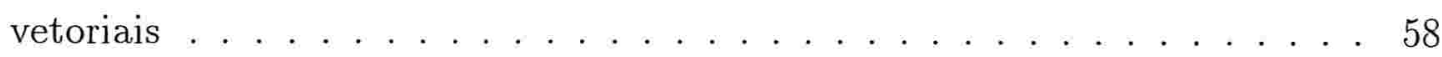

4.3 Operadores de composição compactos entre álgebras de germes holomorfos a valores vetoriais . . . . . . . . . . . . . . . . . 63

4.4 Operadores de composição fracamente compactos entre álgebras de germes holomorfos a valores vetoriais . . . . . . . . . . . . . . 67 


\section{Introdução}

O objetivo deste trabalho é estudar os homomorfismos contínuos entre álgebras de germes de funções holomorfas definidas em um subconjunto compacto de um espaço de Banach munido da topologia de Nachbin $\tau_{w}$.

Os germes de funções holomorfas em subconjuntos compactos de espaços de dimensão finita foi estudado por G. Köthe, A. Grothendieck, A. Martineau e outros. Em [25, 26] L. Nachbin começou estudar o espaço dos germes holomorfos em um compacto de um espaço de Banach e definiu sobre o mesmo a topologia $\tau_{w}$. A partir daí, o espaço vetorial dos germes holomorfos em compactos de espaços localmente convexos, munido de topologias chamadas localmente convexas, passou ser extensivamente estudado tanto do ponto de vista topológico como algébrico. As propriedades deste espaço propiciaram várias aplicações para o estudo do comportamento das funções holomorfas definidas em abertos de espaços localmente convexos, bem como do espaço por elas determinado munido de diferentes topologias. Podemos citar aqui alguns autores que trabalharam nesta direção, tais como S. B. Chae, A. Hirschowitz e J. Mujica.

Em 1981, O. Nicodemi em sua tese de doutorado considerou a estrutura de álgebra topológica determinada nos espaços de germes holomorfos quando munido da topologia compacto aberta. Ela estudou os homomorfismos contínuos entre tais álgebras e apresentou uma caracterização de quando os homomorfismos contínuos são operadores de composição.

Nos pareceu natural a partir do trabalho de Nicodemi, estudar os homomorfismos contínuos entre álgebras de germes holomorfos quando as mesmas estão munidas da to- 
pologia $\tau_{w}$ definida por L. Nachbin.

Como o espaço dos germes tem inúmeras propriedades preservadas pelo limite indutivo, isto nos permite apresentar uma caracterização de quando os homomorfismos contínuos entre álgebras de germes holomorfos são operadores de composição e a partir daí estudar compacidade e compacidade fraca de tais operadores.

No que segue descreveremos os assuntos abordados em cada capítulo.

No capítulo 1 apresentamos algumas definições, notações e resultados relativos a limites indutivos limitadamente retroativos e fortemente limitadamente retroativos. Sejam $E$ um espaço localmente convexo, $K \subset E$ um subconjunto compacto e $\left(\mathcal{H}(K), \tau_{w}\right)$ o espaço dos germes holomorfos munido da topologia de Nachbin $\tau_{w}$. Seja $U$ um aberto de $E$, denotamos por $H^{\infty}(U)$ a álgebra de Banach de todas as funções holomorfas e limitadas em $U$ e por $H_{b}(U)$ a álgebra de Fréchet de todas as funções holomorfas limitadas em subconjuntos $U$-limitados de $U$. Em [19] J. Mujica mostrou que $\left(\mathcal{H}(K), \tau_{w}\right)$ é uma álgebra localmente m-convexa, $\left(\mathcal{H}(K), \tau_{w}\right)=\lim _{n \in \mathbb{N}} H^{\infty}\left(U_{n}\right), \operatorname{com} U_{n}=K+B_{\frac{1}{n}}(0) \subset E$, e que tal limite indutivo é limitadamente retroativo. Usando estes resultados, mostramos na proposição 1.3 .5 que o limite indutivo $\left(\mathcal{H}(K), \tau_{w}\right)=\lim _{n \in \mathbb{N}} H_{b}\left(U_{n}\right)$ é fortemente limitadamente retroativo, quando $K$ é um subconjunto compacto de um espaço de Banach $E$. Como consequência, provamos que para cada $n \in \mathbb{N}$, existe $m \in \mathbb{N}$ tal que as topologias fracas de $\mathcal{H}(K)$ e $H_{b}\left(U_{m}\right)$ coincidem sobre cada limitado de $H_{b}\left(U_{n}\right)$. Tais resultados são de suma importância para estudar os operadores de composição fracamente compactos entre espaços de germes holomorfos.

No capítulo 2, entitulado "Operadores de Composição", apresentamos alguns exemplos e resultados sobre operadores de composição entre certas álgebras de funções holomorfas quando munidas da topologia de Nachbin. Apresentamos definições e exemplos sobre operadores de composição entre álgebras de Fréchet de funções holomorfas definidas em espaços de Banach de dimensão finita. Seja $H(U)$ o espaço de todas as funções holomorfas definidas em um aberto $U$ de um espaço localmente convexo $E$. Em [16] J. M. Isidro mostrou que o espectro das álgebras topológicas $(H(U), \tau) \operatorname{com} \tau=\tau_{0}$ ou $\tau_{w}$, quando $E$ tem a propriedade da aproximação e $U$ é um subconjunto aberto convexo 
e equilibrado esta formado por avaliações. Usando os resultados de Isidro mostramos na proposição 2.2.1 que sob certas condições os homomorfismos contínuos entre estas álgebras topológicas são operadores de composição. Em [21] J. Mujica demonstrou que cada homomorfismo contínuo complexo em $\left(H(U), \tau_{w}\right)$, onde $U$ é um conjunto aberto polinomialmente convexo de um espaço de Fréchet com a propriedade de aproximação, é uma avaliação. Posteriormente, em [22] J. Mujica demonstrou tal resultado para abertos polinomialmente convexos em espaços localmente convexos quase-completos com a propriedade de aproximação. Este resultado de J. Mujica estende o acima citado resultado de J. M. Isidro. Como consequência do referido resultado de J. Mujica, apresentamos na proposição 2.2 .3 condições para que homomorfismos entre estas álgebras de funções holomorfas sejam operadores de composição.

No capítulo 3, estudamos homomorfismos contínuos entre álgebras de germes holomorfos. Demonstramos na primeira seção uma caracterização de quando os homomorfismos contínuos entre álgebras de germes holomorfos são operadores de composição. Apresentamos através dos corolários 3.1.4 e 3.1.5 exemplos de espaços de Banach onde os homomorfismos contínuos entre álgebras de germes holomorfos são operadores de composição. Tais resultados são consequências do teorema 3.1.3, da proposição 3 de [13] e teorema 1.6 de [23]. Na seção seguinte, passamos a estudar os homomorfismos contínuos entre a álgebra de germes holomorfos, $\mathcal{H}(K)$, e a álgebra de Fréchet $H_{b}(U)$ das funções holomorfas de tipo limitado quando munida da topologia da convergência uniforme sobre os $U$-limitados de $U$. As duas últimas seções estão voltadas ao estudo da compacidade e compacidade fraca dos operadores de composição entre álgebras de germes holomorfos. Mostramos também que, sob determinadas condições, os operadores de composição fracamente compactos entre germes holomorfos são compactos.

No capítulo 4, estudamos operadores de composição entre álgebras de germes holomorfos a valores em uma álgebra de Banach. Na primeira seção estudamos operadores de composição compactos e fracamente compactos entre álgebras de Fréchet de funções holomorfas de tipo limitado a valores em uma álgebra de Banach. Em seguida, damos uma condição necessária e suficiente para que os homomorfismos contínuos entre álgebras de germes holomorfos sejam operadores de composição. As últimas duas seções estão 
voltadas ao estudo de operadores de composição compactos e fracamente compactos entre álgebras de germes holomorfos a valores em uma álgebra de Banach. 


\section{Capítulo 1}

\section{Germes de funções holomorfas}

Este capítulo está dividido em 3 seções. Na primeira delas apresentamos as notações que usaremos no decorrer deste trabalho. Na seção seguinte apresentamos algumas definições e resultados relacionados com germes de funções holomorfas. E na última seção apresentamos alguns resultados a respeito de limites indutivos, que nos serão úteis para estudar homomorfismos entre álgebras de germes holomorfos.

\section{$1.1 \quad$ Notações}

Usaremos as notações sobre análise complexa em espaços de Banach e espaços vetoriais topológicos dos livros [7], [8], [18] e [15], [27] respectivamente. A menos que se mencione algo, estaremos utilizando a seguinte notação.

$\mathbb{N}$ o conjunto dos números naturais.

$\mathbb{R}$ o conjunto dos números reais.

$\mathbb{C}$ o conjunto dos números complexos.

$E, F$ espaços localmente convexos sobre $\mathbb{C}$.

$B_{E}$ a bola unitária fechada de $E$, se $E$ é um espaço de Banach sobre $\mathbb{C}$. 
$\stackrel{\circ}{B_{E}}$ a bola unitária aberta de $E$, se $E$ é um espaço de Banach sobre $\mathbb{C}$.

$B_{r}(a)$ a bola aberta de centro $a$ e raio $r$ em $E$, se $E$ é um espaço de Banach sobre $\mathbb{C}$. $\mathcal{L}(E, F)$ o espaço das aplicações lineares contínuas de $E$ em $F$.

$\mathcal{L}_{s}\left({ }^{m} E, F\right)$ o espaço das aplicações m-lineares simétricas contínuas de $E$ em $F$.

$\mathcal{P}_{f}(E)$ o espaço dos polinômios contínuos de tipo finito de $E$ em $\mathbb{C}$, isto é, o subespaço gerado por $\left\{\varphi^{n}: \varphi \in E^{\prime}\right.$ e $\left.n \in \mathbb{N}\right\}$.

$\mathcal{P}\left({ }^{m} E, F\right)$ o espaço dos polinômios m-homogêneos contínuos de $E$ em $F$. Cada $P^{m} \in$ $\mathcal{P}\left({ }^{m} E, F\right)$ é da forma $P^{m}(x)=\widehat{A}^{m}(x, \cdots, x)=\widehat{A}(x)$ onde $A^{m} \in \mathcal{L}_{s}\left({ }^{m} E, F\right)$. Se $F=\mathbb{C}$ escreveremos $\mathcal{P}\left({ }^{m} E\right)$ no lugar de $\mathcal{P}\left({ }^{m} E, \mathbb{C}\right)$.

$\mathcal{P}(E, F)$ o espaço dos polinômios contínuos de $E$ em $F$, isto é, dos polinômios $P=$ $P^{0}+\cdots+P^{m}$ para algum $m \in \mathbb{N}$ onde $P^{k} \in \mathcal{P}\left({ }^{k} E, F\right), \forall k=0,1, \cdots, m$. Se $F=\mathbb{C}$ escreveremos $\mathcal{P}(E)$ no lugar de $\mathcal{P}(E, \mathbb{C})$.

$\mathcal{P}_{w s c}(E)$ o espaço dos polinômios fracamente seqüêncialmente contínuos de $E$ em $\mathbb{C}$ (isto é, $P \in \mathcal{P}_{w s c}(E)$ se para qualquer seqüencia $\left(x_{n}\right)_{n}$ fracamente convergente para $x \in E$, temos que $\left(P\left(x_{n}\right)\right)_{n}$ converge para $\left.P(x) \in \mathbb{C}\right)$.

$H(U)$ a álgebra das funções holomorfas sobre o conjunto aberto $U \subset E$ com valores em $\mathbb{C}$ com as seguintes operações: $(f+g)(x)=f(x)+g(x),(\lambda f)(x)=\lambda f(x),(f g)(x)=$ $f(x) g(x), \forall x \in U, \forall f, g \in H(U), \forall \lambda \in \mathbb{C}$.

$\delta_{x}: H(U) \rightarrow \mathbb{C}$ (para cada $x \in U$ ) a aplicação dada por $\delta_{x}(f)=f(x), \forall f \in H(U)$. A aplicação $\delta_{x}$ é chamada de avaliação em $x$.

$C: E \rightarrow E^{\prime \prime}$ a aplicação natural dada por $C(x)\left(x^{\prime}\right)=x^{\prime}(x), \forall x \in E, \forall x^{\prime} \in E^{\prime}$. Esta aplicação é uma isometria, pois $\|x\|=\|C(x)\|, \forall x \in E$.

$\left(H(U), \tau_{0}\right)$ a álgebra das funções holomorfas sobre o conjunto aberto $U \subset E$ com a topologia compacto aberta $\tau_{0}$. Uma base de vizinhanças abertas de $f_{0} \in H(U)$ é dado por todos os $V_{f_{0}, K, \epsilon}=\left\{f \in H(U): \sup _{x \in K}\left|f(x)-f_{0}(x)\right|<\epsilon\right\}$, para cada $K \subset U$ compacto e $\epsilon>0$. 
$H^{\infty}(U)$ a álgebra de Banach de todas as aplicações holomorfas de $U$ em $\mathbb{C}$ que são limitadas sobre $U$ munida com a norma do supremo sobre $U$, denotada por $\|f\|_{U}=\sup _{x \in U}|f(x)|$.

$\mathcal{B}_{(\mathcal{A}, \tau)}(a)$ seja $\mathcal{A}$ uma álgebra topológica com topologia $\tau$, denotamos por $\mathcal{B}_{(\mathcal{A}, \tau)}(a)$ o conjunto das vizinhanças de $a \in \mathcal{A}$ em $(\mathcal{A}, \tau)$. Sempre que conveniente faremos a seguinte simplificação $\mathcal{B}_{\mathcal{A}}(a)$.

\subsection{Definições e resultados preliminares sobre germes holomorfos}

No decorrer deste trabalho estaremos apresentando resultados a respeito de homomorfismos entre os germes de funções holomorfas. A seguir apresentamos algumas definições que nos serão úteis.

A seguinte definição pode ser encontrada em [4].

Definição 1.2.1 (O espaço $\mathcal{H}(K)$ ). Seja $K$ um subconjunto compacto de um espaço de Banach E. Seja $h(K)=\bigcup\{H(U): U \supset K$ é aberto em $E\}$. Dizemos que duas funções $f, g \in h(K)$ definidas sobre conjuntos abertos $U_{1}$ e $U_{2}$ respectivamente são equivalentes módulo $K$, denotando por $f \sim g$, se existe um conjunto aberto $W \subset E$ com $K \subset W \subset$ $U_{1} \cap U_{2}$ tal que $f_{\left.\right|_{W}}=g_{\left.\right|_{W}}$.

Esta relação $\sim$ define uma relação de equivalência sobre $h(K)$. Dizemos que cada classe de equivalência é um germe de funções holomorfas em $K$ (ou germe holomorfo em $K)$. Denotamos por $[f]$ a classe de equivalência módulo $K$ determinada por $f$.

Denotamos por $\mathcal{H}(K)$ o espaço quociente de $h(K)$ com relação a $\sim \mathcal{H}(K)$ é uma álgebra sobre $\mathbb{C}$ com as operaçôes $[f]+[g]=[f+g],[\lambda f]=\lambda[f]$ e $[f] \cdot[g]=[f \cdot g]$ para cada $f, g \in h\left(K^{r}\right)$ e $\lambda \in \mathbb{C}$.

A seguir daremos a definição da topologia de Nachbin que será uma topologia natural, a ser considerada, no espaço de germes de funções holomorfas. 
Definição 1.2.2 (topologia de Nachbin $\tau_{w}$ ). Sejam $E$ um espaço de Banach e $U \subset E$ um conjunto aberto. Uma semi-norma p sobre $H(U)$ é portada por um subconjunto compacto $K \subset U$ se para cada subconjunto aberto $V$, com $K \subset V \subset U$, existe $c(V)>0$ tal que

$$
p(f) \leq c(V) \sup _{x \in V}|f(x)|, \forall f \in H(U)
$$

A topologia de Nachbin, que denotaremos por $\tau_{w}$, é a topologia localmente convexa sobre $H(U)$ definida por todas as semi-normas portadas por algum subconjunto compacto $K \subset U$.

Para obter resultados com relação aos homomorfismos entre germes de funções holomorfos, faremos uso da álgebra das funções holomorfas de tipo limitado definida em conjuntos $U$-limitados. Para tal vamos introduzir alguns conceitos.

Definição 1.2.3 Sejam $E$ um espaço de Banach e $U \subset E$ um subconjunto aberto. Dizemos que um conjunto $A \subset U$ é $U$-limitado se $A$ é limitado e $d(A, \partial U)>0$, onde $d$ é a função distancia e $\partial U$ é a fronteira de $U$.

Denotamos por $H_{b}(U)$ a álgebra de Fréchet de todas as aplicações holomorfas de $U$ em $\mathbb{C}$ que são limitadas sobre conjuntos U-limitados munida da topologia da convergência uniforme $\tau_{b}$ sobre os U-limitados.

A seguir daremos a definição de limites indutivos de espaços localmente convexos.

Definição 1.2.4 (sistema indutivo e limite indutivo). Sejam I um conjunto dirigido (sob uma ordem $\leq)$ e, $\left(E_{i}, \tau_{i}\right)_{i \in I}$ uma família de espaços localmente convexos $E_{i}$ sobre $\mathbb{C}$ com topologias $\tau_{i}$ tais que, se $i \leq j, E_{i}$ é subespaço vetorial de $E_{j}$ e a inclusão canônica $f_{j i}:\left(E_{i}, \tau_{i}\right) \rightarrow\left(E_{j}, \tau_{j}\right)$ é contínua (aqui, $f_{i i}: E_{i} \rightarrow E_{i}$ é a aplicação identidade). Então $\left(E_{i}, \tau_{i}\right)_{i \in I}$ é chamado de sistema indutivo (injetivo) de espaços localmente convexos.

Um espaço localmente $(E, \tau)$ é um limite indutivo (localmente convexo) do sistema $\left(E_{i}, \tau_{i}\right)_{i \in I}$ se para cada $i \in I$ existe uma aplicação linear $f_{i}: E_{i} \rightarrow E$ tal que 
$f_{i}=f_{j} \circ f_{j i}, \forall j \geq i, E=\bigcup_{i \in I} f_{i}\left(E_{i}\right)$ e E está munido da topologia localmente convexa mais fina que torna todas as aplicações $f_{i}:\left(E_{i}, \tau_{i}\right) \rightarrow E$ contínuas.

Se $(E, \tau)$ é o limite indutivo localmente convexo do sistema $\left(E_{i}, \tau_{i}\right)_{i \in I}$ em relação as aplicações $\left(f_{i}\right)_{i \in I}$, denotamos $(E, \tau)=\lim _{\vec{i} \in I}\left(E_{i}, \tau\right)$ ou, simplesmente, $E=\underset{\overrightarrow{i \in I}}{\lim _{i \rightarrow I}} E_{i}$.

Definição 1.2.5 Seja $\mathcal{U}$ a classe dos subconjuntos abertos $U$ de um espaço de Banach $E$ tais que $U \supset K$ (onde $K$ é um subconjunto compacto fixado). Consideremos em $\mathcal{U}$ a seguinte ordem: $U \leq V \Leftrightarrow V \subset U$. Para cada $U \in \mathcal{U}$, seja $\mathcal{I}_{U}^{K}: H(U) \rightarrow \mathcal{H}(K)$ a aplicação definida por $\mathcal{I}_{U}^{K}(f)=[f]$.

(a) $\left(\mathcal{H}(K), \tau_{0}\right)$ é o limite indutivo do sistema $\left(H(U), \tau_{0}\right)_{U \in \mathcal{U}}$ com relação as aplicações $\left(\mathcal{I}_{U}^{K}\right)_{U \in \mathcal{U}}$. Dizemos que $\left(\mathcal{H}(K), \tau_{0}\right)=\lim _{\overline{U \supset K}}\left(H(U), \tau_{0}\right)$ é o espaço de germes de funções holomorfas em $K$ com a topologia compacto aberta $\tau_{0}$.

(b) $\left(\mathcal{H}(K), \tau_{w}\right)$ é o limite indutivo do sistema $\left(H(U), \tau_{w}\right)_{U \in \mathcal{U}}$ com relação as aplicações $\left(\mathcal{I}_{U}^{K}\right)_{U \in \mathcal{U}}$. Dizemos que $\left(\mathcal{H}(K), \tau_{w}\right)=\lim _{\overrightarrow{U \supset \hat{k}}}\left(H(U), \tau_{w}\right)$ é o espaço de germes de funções holomorfas em $K$ com a topologia de Nachbin $\tau_{w}$.

Observações 1.2.6 Seja $\mathcal{H}(K)$ o limite indutivo da definição 1.2.5.

1. A definição de $\mathcal{H}(K)$ não muda se nos restringirmos a subconjuntos abertos $U \in \mathcal{U}$ cujas componentes conexas tem interseção não vazia com $K$ (consideramos também o sistema fundamental de vizinhanças de $K$ dado por $\left.U_{n}=K+B_{\frac{1}{n}}(0) \subset E\right)$. Assim, as aplicações $\mathcal{I}_{U}^{K}: H(U) \rightarrow \mathcal{H}(K)$ são injetivas pela unicidade do prolongamento analítico, e identificamos neste caso $H(U)$ como um subespaço vetorial de $\mathcal{H}(K)$, desta forma $\mathcal{H}(K)=\bigcup_{U \in \mathcal{U}} H(U)$ e $\mathcal{H}(K)=\lim _{\overrightarrow{U \supset K}} H(U)$.

Daqui em diante estamos considerando este limite indutivo.

2. Quando $K=\{c\}$, denotaremos $\mathcal{H}(K)$ por $\mathcal{H}(c)$. 
Proposição 1.2.7 Sejam E um espaço de Banach complexo $K \subset E$ um compacto. Então

(a) $\left(\mathcal{H}(K), \tau_{w}\right)$ é uma álgebra localmente m-convexa.

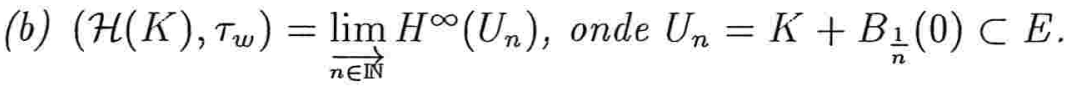

Demonstração. Ver J. Mujica [19, pgs. 81, 163].

Observações 1.2.8 1. Se $K$ é convexo e/ou equilibrado então $U_{n}$ é convexo e/ou equilibrado.

2. Dado que $H^{\infty}\left(U_{n}\right) \hookrightarrow H_{b}\left(U_{n}\right) \hookrightarrow\left(\mathcal{H}(K), \tau_{w}\right)$ temos que $\left(\mathcal{H}(K), \tau_{w}\right)=\underset{n \in \mathbb{\mathbb { N }}}{\lim _{b}} H_{b}\left(U_{n}\right)$, onde $\mathcal{I}_{n}^{K}: H_{b}\left(U_{n}\right) \hookrightarrow \mathcal{H}(K)$ é a inclusão natural e denotaremos por $[f]=\mathcal{I}_{n}^{K}(f), \forall f \in H_{b}\left(U_{n}\right)$, a classe de equivalência determinado por $f$.

Vamos estudar no decorrer deste trabalho os operadores de composição entre as álgebras $H(U)$ e $\mathcal{H}(K)$. Para tal, apresentamos a seguir as definições de operadores de composição entre estas álgebras.

Definição 1.2.9 Sejam E, $F$ espaços de Banach, $U \subset E$ e $V \subset F$ conjuntos abertos. Um homomorfismo $T: H(U) \rightarrow H(V)$ é um operador de composição, se existe uma aplicação $g \in H(V, E)$ com $g(V) \subset U$ tal que $T(f)=f \circ g, \forall f \in H(U)$. Denotamos o operador de composição $T$ por $T_{g}$.

Definição 1.2.10 Sejam $E, F$ espaços de Banach complexos, $K \subset E$ e $L \subset F$ subconjuntos compactos. Um homomorfismo $A: \mathcal{H}(K) \rightarrow \mathcal{H}(L)$ é um operador de composição, se existem um aberto $V \supset L$ e uma função holomorfa $\Phi: V \rightarrow E$ tais que $\Phi(L) \subset K$ e $A([f])=[f \circ \Phi]$ para cada função holomorfa $f$ definida sobre uma vizinhança de $K$. Denotamos o operador de composição $A$ por $A_{\Phi}$.

Em [28] Nicodemi apresentou tal definição e estudou os homomorfismos contínuos entre germes de funções holomorfas.

No decorrer deste trabalho, vamos precisar dos seguintes dois resultados. 
Teorema 1.2.11 (A. Grothendieck). Sejam E um espaço localmente convexo de Hausdorff, $\left(E_{i}\right)_{i}$ uma seqüência de espaços de Fréchet; e $T_{i}: E_{i} \rightarrow E$ aplicações lineares contínuas para cada $i$. Sejam $F$ um espaço de Fréchet $e T: F \rightarrow E$ uma aplicação linear contínua, tal que $T(F) \subset \bigcup_{i} T_{i}\left(E_{i}\right)$. Então existe um indice $j$ tal que $T(F) \subset T_{j}\left(E_{j}\right)$ e, se $T_{j}$ é injetora, podemos escrever $T=T_{j} \circ S$, onde $S: F \rightarrow E_{j}$ é uma aplicação linear contínua.

Demonstração. Veja a demonstração em [14], teorema(A) pg.16.

Proposição 1.2.12 (Lema de aproximação de Grothendieck) Sejam E um espaço localmente convexo, Hausdorff, $E^{\prime}$ o espaço dual e $B$ um subconjunto convexo, equilibrado e fechado de $E$. Seja $f: E \rightarrow \mathbb{C}$ uma forma linear tal que $f_{\left.\right|_{B}}: B \rightarrow \mathbb{C}$ é contínua. Então para cada $\epsilon>0$ existe $g \in E^{\prime}$ tal que $|f(x)-g(x)| \leq \epsilon, \forall x \in B$.

Demonstração. Veja a demonstração em [15], proposição 1 pg. 248.

\subsection{Algumas propriedades de limites indutivos}

No capítulo 3 vamos caracterizar operadores de composição compactos e fracamente compactos entre os espaços de germes holomorfos. Para tais caracterizações vamos precisar de algumas propriedades sobre $\mathcal{H}(K)$.

As seguintes definições podem ser encontradas em [3]. Observamos que daqui para a frente, sempre que dissermos que $(H, \tau)=\underset{\overrightarrow{i \in I}}{\lim }\left(H_{i}, \tau_{i}\right)$ estaremos supondo $H=\bigcup_{i \in I} H_{i}$ e que o limite indutivo é tomado com relação às incluções $H_{i} \hookrightarrow H$. Denotaremos por $(H, \tau)_{\left.\right|_{B}}$ a topologia $\tau_{\left.\right|_{B}}$ para $B \subset H$.

Definições 1.3.1 Seja $\left(H_{i}, \tau_{i}\right)_{i \in I}$ uma família de espaços localmente convexos e $(H, \tau)=$ $\lim _{i \in I}\left(H_{i}, \tau_{i}\right)$ o limite indutivo.

1. Dizemos que $(H, \tau)$ é um limite indutivo regular: se para cada conjunto limitado $B$ em $(H, \tau)$, existe $i \in I$ tal que $B \subset H_{i}$ e $B$ é $\tau_{i}$-limitado. 
2. Dizemos que $(H, \tau)$ é limitadamente retroativo: se para cada subconjunto limitado $B$ de $(H, \tau)$ existe $i \in I$ tal que $B \subset\left(H_{i}, \tau_{i}\right)$ é limitado e $(H, \tau)_{\left.\right|_{B}}=\left(H_{i}, \tau_{i}\right)_{\left.\right|_{B}}$

3. Dizemos que $(H, \tau)$ é fortemente limitadamente retroativo se:

(a) $(H, \tau)$ é um limite indutivo regular,

(b) Para cada $i \in I$, existe $j \geq i$ tal que $(H, \tau)_{\left.\right|_{B}}=\left(H_{j}, \tau_{j}\right)_{\left.\right|_{B}}$ para cada subconjunto limitado $B$ de $\left(H_{i}, \tau_{i}\right)$.

Observações 1.3.2 1. Os limites indutivos limitadamente retroativos e fortemente limitadamente retroativos são limites indutivos regulares.

2. Claramente, limites indutivos fortemente limitadamente retroativos são limitadamente retroativos,

3. Limites indutivos enumeráveis de espaços normados são limitadamente retroativos se, e somente se, são fortemente limitadamente retroativos.

É facil verificar as observaçôes dos itens (1) e (2). Vamos demonstrar aqui o item (3) da observação. Para tal é suficiente mostrar que limitadamente retroativo implica fortemente limitadamente retroativo. Seja $i \in \mathbb{N}$ e $B_{H_{i}}$ a bola unitária fechada de $\left(H_{i}, \tau_{i}\right)$, como $(H, \tau)$ é limitadamente retroativo existe $k \in I$ tal que $B_{H_{i}} \subset H_{k} e$ $(H, \tau)_{\left.\right|_{B_{H_{i}}}}=\left(H_{k}, \tau_{k}\right)_{\left.\right|_{B_{H_{i}}}}$. Temos dois casos:

(a) se $k>i$, considere $j=k \operatorname{assim} j>i$ e $(H, \tau)_{\left.\right|_{B_{H_{i}}}}=\left(H_{j}, \tau_{j}\right)_{\left.\right|_{B_{H_{i}}}}$.

(b) se $k\left\langle i\right.$, seja $j>i>k$ logo temos as seguintes inclusões $f_{i k}: H_{k} \rightarrow H_{i}$, $f_{j i}: H_{i} \rightarrow H_{j}, f_{j k}: H_{k} \rightarrow H_{j}, f_{j}: H_{j} \rightarrow H$ e $f_{k}: H_{k} \rightarrow H$ tais que $f_{j k}=f_{j i} \circ f_{i k}, f_{k}=f_{j} \circ f_{j k}$. Como $B_{H_{i}} \subset H_{k}$ pela inclusão $f_{j k}$ temos que $B_{H_{i}} \subset H_{j}$.

Considere $A \subset B_{H_{i}}$. Mostraremos que $\bar{A}^{\tau}=\bar{A}^{\tau_{j}}$. Claramente, $\bar{A}^{\tau_{j}} \subset \bar{A}^{\tau}$. Por outro lado, seja $x \in \bar{A}^{T}$, então existe uma rede $\left(x_{\alpha}\right)_{\alpha} \subset A$ tal que $x_{\alpha} \stackrel{\tau}{\rightarrow} x$, como $(H, \tau)_{\left.\right|_{B_{H_{i}}}}=\left(H_{k}, \tau_{k}\right)_{\left.\right|_{B_{H_{i}}}}$, temos que $x_{\alpha} \stackrel{\tau_{k}}{\rightarrow} x$ e pela continuidade da inclusão $f_{j k}$ temos que $x_{\alpha} \stackrel{\tau_{j}}{\rightarrow} x$, logo $x \in \bar{A}^{\tau_{j}}$. Portanto, $\bar{A}^{\tau}=\bar{A}^{\tau_{j}}$.

Assim, existe $j>i$ tal que $(H, \tau)_{\left.\right|_{B_{H_{i}}}}=\left(H_{j}, \tau_{j}\right)_{\left.\right|_{B_{i}}}$. 
Dos itens (a) e (b) concluimos que, existe $j>i$ tal que $\left.(H, \tau)\right|_{B_{H_{i}}}=\left(H_{j}, \tau_{j}\right)_{\left.\right|_{B_{H_{i}}}}$. Agora, seja $\mathcal{X} \subset H_{i}$ um conjunto limitado, logo existe $\lambda>0$ tal que $\mathcal{X} \subset \lambda B_{H_{i}}$, consequentemente $(H, \tau)_{\left.\right|_{\mathcal{X}}}=\left(H_{j}, \tau_{j}\right)_{\left.\right|_{\mathcal{X}}}$.

Proposição 1.3.3 Sejam $E$ um espaço de Banach e $K \subset E$ um subconjunto compacto,

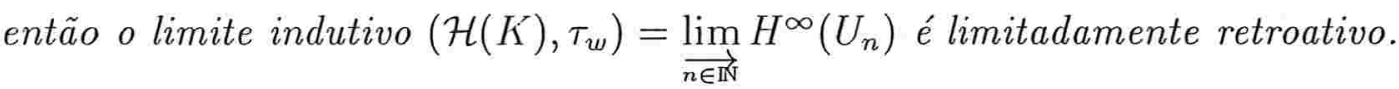

Demonstração. Ver J. Mujica [19, teorema 18.1 pg. 126] ou [20, teorema 3.2 pg. 16].

Proposição 1.3.4 Sejam $\left(H_{i}, \tau_{i}\right)_{i \in \mathbb{N}}$ uma familia de espaços localmente convexos e $(H, \tau)=$ $\lim _{i \in \mathbb{N}}\left(H_{i}, \tau_{i}\right)$ o limite indutivo fortemente limitadamente retroativo. Então para cada $m \in \mathbb{N}$ existe $n>m$ tal que $\sigma\left(H, H^{\prime}\right)_{\left.\right|_{B}}=\sigma\left(H_{n}, H_{n}^{\prime}\right)_{\left.\right|_{B}}$ para cada subconjunto limitado $B$ de $\left(H_{m}, \tau_{m}\right)$.

Demonstração. Seja $B \subset\left(H_{m}, \tau_{m}\right)$ um subconjunto limitado. Da proposição 4.4 de [15, pg. 87], segue que a envoltória absolutamente convexa de $B$, que denotamos por $\Gamma(B)$, é limitada e consequentemente $\overline{\Gamma(B)}$ é absolutamente convexa, fechada e limitada. Como $(H, \tau)$ é fortemente limitadamente retroativo, existe $n>m$ tal que

$$
(H, \tau)_{\left.\right|_{\overline{\Gamma(B)}}}=\left(H_{n}, \tau_{n}\right)_{\left.\right|_{\Gamma(B)}}
$$

Agora, pelo lema de aproximação de Grothendieck (ver 1.2.12) temos que para cada $f \in H^{\prime}$ e para cada $\epsilon>0$, existe $g \in H_{n}^{\prime} \operatorname{com}|f(x)-g(x)|<\epsilon, \forall x \in \overline{\Gamma(B)}$. Por um resultado de De Wilde [33, lema 4 da pg. 161], temos que

$$
\sigma\left(H, H^{\prime}\right)_{\overline{\Gamma(B)}}=\sigma\left(H_{n}, H_{n}^{\prime}\right)_{\overline{\Gamma(B)}}
$$

Portanto, $\sigma\left(H, H^{\prime}\right)_{\left.\right|_{B}}=\sigma\left(H_{n}, H_{n}^{\prime}\right)_{\left.\right|_{B}}$ para cada subconjunto limitado $B$ de $\left(H_{m}, \tau_{m}\right)$.

Na seguinte proposição mostraremos que $\mathcal{H}(K)=\lim _{n \in \mathbb{N}} H_{b}\left(U_{n}\right)$ é fortemente limitadamente retroativo e este resultado será usado na demonstração do teorema 3.3.2. 
Proposição 1.3.5 Sejam $E$ um espaço de Banach e $K \subset E$ um conjunto compacto, então o limite indutivo $\mathcal{H}(K)=\lim _{n \in \mathbb{N}} H_{b}\left(U_{n}\right)$ é fortemente limitadamente retroativo.

Demonstração. Consideremos $\mathcal{H}(K)$ como o limite indutivo $\left(\mathcal{H}(K), \tau_{w}\right)=\underset{\overrightarrow{n \in \mathbb{N}}}{\lim } H^{\infty}\left(U_{n}\right)$. Da proposição 1.3 .3 sabemos que o limite indutivo $\left(\mathcal{H}(K), \tau_{w}\right)=\underset{\lim _{n \in \mathbb{N}}}{ } H^{\infty}\left(U_{n}\right)$ é limitadamente retroativo e pela observação 3 de 1.3 .2 temos que

$$
\left(\mathcal{H}(K), \tau_{w}\right)=\underset{n \in \mathbb{N}}{\lim _{n \rightarrow}} H^{\infty}\left(U_{n}\right) \text { é fortemente limitadamente retroativo }
$$

Agora, mostraremos que o limite indutivo $\mathcal{H}(K)=\underset{n \in \mathbb{\mathbb { N }}}{\lim _{b}} H_{b}\left(U_{n}\right)$ é fortemente limitadamente retroativo, uma vez que vale as seguintes condições:

(a) O limite indutivo $\mathcal{H}(K)=\underset{\overrightarrow{n \in \mathbb{T}}}{\lim _{b}} H_{b}\left(U_{n}\right)$ é regular.

Seja $\mathcal{X} \subset \mathcal{H}(K)$ um conjunto limitado. Por $(1.1)$ temos que $\left(\mathcal{H}(K), \tau_{w}\right)=\lim _{n \in \mathbb{N}} H^{\infty}\left(U_{n}\right)$ é regular, logo existe $m \in \mathbb{N}$ tal que $\mathcal{X} \subset H^{\infty}\left(U_{m}\right)$. Como $H^{\infty}\left(U_{m}\right) \stackrel{i_{m}}{\hookrightarrow} H_{b}\left(U_{m}\right)$, temos que $\mathcal{X} \subset H_{b}\left(U_{m}\right)$ e é limitado.

(b) Sejam $m \in \mathbb{N}$ e $\mathcal{X} \subset H_{b}\left(U_{m}\right)$ um conjunto limitado. Logo $\mathcal{I}_{m}^{K}(\mathcal{X}) \subset \mathcal{H}(K)$ é limitado. Por (1.1) existe $n>m$ tal que $\mathcal{X} \subset H^{\infty}\left(U_{n}\right)$ e $\left(\mathcal{H}(K), \tau_{w}\right)_{\left.\right|_{\mathcal{X}}}=H^{\infty}\left(U_{n}\right)_{\left.\right|_{\mathcal{X}}}$. Agora, considerando as inclusões naturais vamos ter o seguinte diagrama comutativo.

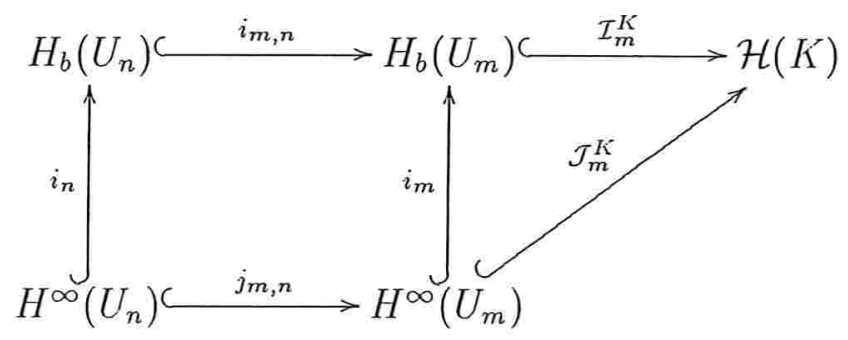

pela continuidade da inclusão $i_{n}$ temos que $\left(\mathcal{H}(K), \tau_{w}\right)_{\left.\right|_{\mathcal{X}}}=H_{b}\left(U_{n}\right)_{\left.\right|_{\mathcal{X}}}$.

Considere $A \subset \mathcal{X} \subset H_{b}\left(U_{m}\right)$. Mostramos que $\bar{A}^{\tau_{w}}=\bar{A}^{\tau_{b}^{n}}$ onde $\tau_{b}^{n}$ é a topologia de $H_{b}\left(U_{n}\right)$. Claramente, $\bar{A}^{\tau_{b}^{n}} \subset \bar{A}^{\tau_{w}}$. Por outro lado, seja $f \in \bar{A}^{\tau_{w}}$, então existe uma rede $\left(f_{\alpha}\right)_{\alpha} \subset A$ tal que $f_{\alpha} \stackrel{\tau_{\varkappa}}{\rightarrow} f$, como $\left(\mathcal{H}(K), \tau_{w}\right)_{\left.\right|_{\chi}}=H_{b}\left(U_{n}\right)_{\left.\right|_{\mathcal{X}}}$, temos que $f_{\alpha} \stackrel{\tau_{b}^{n}}{\rightarrow} f, \operatorname{logo} f \in \bar{A}^{\tau_{b}^{n}}$. Portanto, 
$\bar{A}^{\tau_{w}}=\bar{A}^{\tau_{b}^{n}}$. Assim, o limite indutivo $\mathcal{H}(K)=\underset{\lim _{n \in \mathbb{N}}}{H_{b}}\left(U_{n}\right)$ é fortemente limitadamente retroativo.

O corolário que demonstraremos a seguir será usado na demonstração do teorema 3.4.1 que trata de operadores de composição fracamente compactos entre germes de funções holomorfas.

Corolário 1.3.6 Sejam E um espaço de Banach, $K \subset E$ um subconjunto compacto $e$ o limite indutivo $\mathcal{H}(K)=\lim _{n \in \mathbb{N}} H_{b}\left(U_{n}\right)$. Então, para cada $n \in \mathbb{N}$, existe $m>n$, tal que $\sigma\left(\mathcal{H}(K), \mathcal{H}(K)^{\prime}\right)_{\left.\right|_{\mathcal{X}}}=\sigma\left(H_{b}\left(U_{m}\right), H_{b}\left(U_{m}\right)^{\prime}\right)_{\left.\right|_{\mathcal{X}}}$ para cada subconjunto limitado $\mathcal{X} \subset H_{b}\left(U_{n}\right)$.

Demonstração. O resultado segue diretamente das proposições 1.3.4 e 1.3.5. 


\section{Capítulo 2}

\section{Operadores de composição}

Neste capítulo estudamos operadores de composição entre os espaços de germes de funções holomorfas definidos em subconjuntos compactos de espaços de Banach de dimensão finita. Na seção 2.2 apresentamos uma caracterização dos homomorfismos contínuos entre álgebras do tipo $\left(H(U), \tau_{w}\right)$.

Em [28] O. Nicodemi definiu operadores de composição entre os espaços de germes holomorfos e demonstrou que sob certas condições, homomorfismos contínuos entre álgebras de germes holomorfos munidas da topologia compacto aberta são operadores de composição. Ela fez um comentário de quando $E$ e $F$ são espaços de dimensão finita, $K$ e $L$ conjuntos reduzidos a um ponto, um homomorfismo local é um operador de composição. Dedicamos a seção 1 deste capítulo para demonstrar tal afirmação.

\subsection{Homomorfismos contínuos entre álgebras de ger- mes holomorfos em espaços de dimensão finita}

Nesta seção estudamos alguns casos especiais de operadores de composição entre espaços de germes de funções holomorfas definidas em espaços de Banach $E$ de dimensão finita.

Na proposição 2.1.8, mostramos que sob certas condições todo homomorfismo contínuo 
$A:\left(\mathcal{H}(K), \tau_{w}\right) \rightarrow\left(\mathcal{H}(L), \tau_{w}\right)$ é um operador de composição. Para tal, vamos precisar das seguintes definições.

Definições 2.1.1 Sejam E um espaço de Banach e $K \subset E$ um subconjunto compacto. Seja $\widehat{K}_{\mathcal{P}(E)}=\left\{x \in E:|P(x)| \leq\|P\|_{K}, \forall P \in \mathcal{P}(E)\right.$ onde $\left.\|P\|_{K}=\sup _{x \in K}|P(x)|\right\}$

(a) Dizemos que $K$ é polinomialmente convexo se $\widehat{K}_{\mathcal{P}(E)}=K$.

(b) Dizemos que um conjunto aberto $U \subset E$ é polinomialmente convexo se $\widehat{K}_{\mathcal{P}(E)} \cap U$ é compacto para cada compacto $K \subset U$.

\section{Exemplos 2.1.2}

(a) Seja $E=\mathbb{C}$ o corpo complexo e $K=\left\{k_{1}, k_{2}\right\}$ um subconjunto de $E$, então $K$ é polinomialmente convexo.

Como $K \subset \widehat{K}_{\mathcal{P}(E)}$, basta mostrar que $\widehat{K}_{\mathcal{P}(E)} \subset K$. Seja $x_{0} \in \widehat{K}_{\mathcal{P}(E)}$. Então $\left|P\left(x_{0}\right)\right| \leq\|P\|_{K}, \forall P \in \mathcal{P}(E)$. Consideremos o polinômio $Q \in \mathcal{P}\left({ }^{2} E\right)$ dado por $Q(x)=\left(x-k_{1}\right)\left(x-k_{2}\right)$. Então $\left|Q\left(x_{0}\right)\right| \leq\|Q\|_{K}=0$ e assim $Q\left(x_{0}\right)=0$. Desta forma $x_{0}=k_{1}$ ou $k_{2}$, consequentemente $x_{0} \in K$. Portanto, $\widehat{K}_{\mathcal{P}(E)} \subset K$.

Observamos que um argumento similar mostra que $K=\left\{k_{1}, k_{2}, \cdots, k_{n}\right\}$ é exemplo de conjunto polinomialmente convexo.

(b) Sejam E um espaço de Banach e $U \subset E$ um subconjunto aberto convexo, então $U$ é polinomialmente convexo.

Este é um caso particular da proposição 2.7 de [22].

Definição 2.1.3 Seja E um espaço localmente convexo, Hausdorff e completo. Dizemos que E tem a propriedade de aproximação, se para cada vizinhança de zero $V$ em $E$, e cada subconjunto compacto, $K \subset E$, existe uma aplicação linear contínua $T: E \rightarrow E$ com $\operatorname{dim}(T(E))<\infty$ tal que $T(x)-x \in V, \forall x \in K$. 
A seguir demonstraremos a seguinte afirmação feita por O. Nicodemi em [28]: Quando $E$ e $F$ são espaços de Banach de dimensão finita, cada homomorfismo local contínuo será um operador de composição. Para tal precisamos introduzir alguns conceitos.

Definições 2.1.4 Sejam $\mathcal{A}$ e $\mathcal{B}$ anéis comutativos.

(a) Dizemos que $\mathcal{A}$ é um anel local se $\mathcal{A}$ possui um único ideal maximal $\mathcal{M}$. Denotaremos este anel local por $(\mathcal{A}, \mathcal{M})$.

(b) Dizemos que um homomorfismo $\psi:(\mathcal{A}, \mathcal{M}) \rightarrow(\mathcal{B}, \mathcal{N})$ entre anéis locais é um homomorfismo local se $\psi(\mathcal{M}) \subset \mathcal{N}$.

Exemplo 2.1.5 Sejam E um espaço de Banach de dimensão finita e $c \in E$, então $\left(\mathcal{H}(c), \tau_{0}\right)$ é um anel local.

Para demonstrar tal afirmação vamos considerar o seguinte limite indutivo $\left(\mathcal{H}(c), \tau_{0}\right)=$ $\underset{n \in \mathbb{N}}{\lim }\left(H\left(B_{\frac{1}{n}}(c)\right), \tau_{0}\right)$ e vamos introduzir a seguinte notação:

Para cada $n \in \mathbb{N}, \mathcal{I}_{n}^{c}: H\left(B_{\frac{1}{n}}(c)\right) \hookrightarrow \mathcal{H}(c)$ a inclusão natural $\mathcal{I}_{n}^{c}(f)=[f], \forall f \in$ $H\left(B_{\frac{1}{n}}(c)\right), 1_{n}^{c}$ denota a unidade da álgebra $H\left(B_{\frac{1}{n}}(c)\right)$ e $1^{c}=\left[1_{n}^{c}\right]$ denota a unidade dos germes $\left(\mathcal{H}(c), \tau_{0}\right)$.

No que segue mostraremos que $\mathcal{M}_{c}=\{[f] \in \mathcal{H}(c): f(c)=0\}$ é o único ideal maximal de $\mathcal{H}(c)$. É claro que $\mathcal{M}_{c}$ é um ideal. Vamos primeiramente mostrar que $\mathcal{M}_{c}$ é maximal. Para tal, seja $\mathcal{I}$ um ideal de $\mathcal{H}(c) \operatorname{com} \mathcal{M}_{c} \varsubsetneqq \mathcal{I} \subset \mathcal{H}(c)$. Logo existe $[f] \in \mathcal{I}$ tal que $[f] \notin \mathcal{M}_{c}$, isto é $f(c) \neq 0$. Assim existe $m>0$ com $f(x) \neq 0, \forall x \in B_{\frac{1}{m}}(c)$. Consideremos, $g(x)=\frac{1}{f(x)}, \forall x \in B_{\frac{1}{m}}(c)$, então $[g] \in \mathcal{H}(c)$. Como $\mathcal{I}$ é um ideal temos que $[f][g]=\left[1^{c}\right] \in \mathcal{I}$, consequentemente, $\mathcal{I}=\mathcal{H}(c)$ e $\mathcal{M}_{c}$ é maximal.

Finalmente, vamos mostrar que o ideal maximal $\mathcal{M}_{c}$ é único. De fato, vamos supor que existem dois ideais maximais distintos $\mathcal{M}$ e $\mathcal{M}_{c}$. Assim, existe $[f] \in \mathcal{M}$ com $[f] \notin$ $\mathcal{M}_{c}$, isto é $f(c) \neq 0$. Consequentemente existe $m>0$ tal que $f(x) \neq 0, \forall x \in B_{\frac{1}{m}}(c)$. Consideremos, $g(x)=\frac{1}{f(x)}, \forall x \in B_{\frac{1}{m}}(c)$, então $[g] \in \mathcal{H}(c)$ e como $\mathcal{M}$ é ideal temos que $[f][g]=\left[1^{c}\right] \in \mathcal{M}, \log o \mathcal{M}=\mathcal{H}(c)$ (absurdo!). Portanto, $\mathcal{M}=\mathcal{M}_{c}$. 
Proposição 2.1.6 Sejam $E, F$ espaços de Banach de dimensão finita, $c \in E, d \in F$ e $r, s>0$. Então cada homomorfismo contínuo $\psi:\left(H\left(B_{r}(c)\right), \tau_{0}\right) \rightarrow\left(H\left(B_{s}(d)\right), \tau_{0}\right)$ é um operador de composição.

Demonstração. Vamos mostrar que existe uma aplicação holomorfa $\Phi: B_{s}(d) \rightarrow B_{r}(c)$ tal que $\psi(f)=f \circ \Phi, \forall f \in H\left(B_{r}(c)\right)$.

De fato, sejam $y \in B_{s}(d)$ e o homomorfismo comtínuo $\delta_{y}:\left(H\left(B_{s}(d)\right), \tau_{0}\right) \rightarrow \mathbb{C}$ logo $\delta_{y} \circ \psi:\left(H\left(B_{r}(c)\right), \tau_{0}\right) \rightarrow \mathbb{C}$ é um homomorfismo contínuo, então existe um conjunto compacto $K \subset B_{r}(c)$ tal que

$$
\left|\delta_{y} \circ \psi(f)\right| \leq\|f\|_{K}, \forall f \in H\left(B_{r}(c)\right) .
$$

Agora, $\left(\delta_{y} \circ \psi\right)_{\left.\right|_{E^{\prime}}}:\left(E^{\prime}, \tau_{0}\right) \rightarrow \mathbb{C}$ é linear contínuo e como $E$ tem dimensão finita $\left(\delta_{y} \circ \psi\right)_{\left.\right|_{E^{\prime}}} \in E^{\prime \prime}=E$, assim existe um único $x(y) \in E$ tal que

$$
\delta_{y} \circ \psi\left(x^{\prime}\right)=x^{\prime}(x(y)), \forall x^{\prime} \in E^{\prime} .
$$

Dado que $\delta_{y} \circ \psi$ é um homomorfismo e $E$ tem dimensão finita de (2.2) temos que $\delta_{y} \circ \psi(P)=P(x(y)), \forall P \in \mathcal{P}(E)=\mathcal{P}_{f}(E)$, e por (2.1) segue que

$$
|P(x(y))| \leq\|P\|_{K}, \forall P \in \mathcal{P}(E),
$$

consequentemente $x(y) \in \widehat{K}_{\mathcal{P}(E)}$. Como $B_{r}(c)$ é convexo, pela proposição 2.7 de [22], temos que $\widehat{K}_{\mathcal{P}(E)} \subset B_{r}(c), \operatorname{logo} x(y) \in B_{r}(c)$.

Assim, definimos uma aplicação $\Phi: B_{s}(d) \rightarrow B_{r}(c)$ por $\Phi(y)=x(y), \forall y \in B_{s}(d)$. Por (2.2) temos que $\psi(P)=P \circ \Phi, \forall P \in \mathcal{P}(E)$. Como $E$ tem dimensão finita e $B_{r}(c)$ é polinomialmente convexo (ver exemplo 2.1.2 (c)), segue pelo teorema 25.4 de [18], que

$$
\psi(f)=f \circ \Phi, \forall f \in H\left(B_{r}(c)\right)
$$

e além disso, $\Phi \in H\left(B_{s}(d), E\right)$, uma vez que $\left(E^{\prime},\|\|\right) \hookrightarrow\left(H\left(B_{r}(c)\right), \tau_{0}\right)$ e por (2.3) temos que $x^{\prime} \circ \Phi=\psi\left(x^{\prime}\right) \in H\left(B_{s}(d)\right), \forall x^{\prime} \in E^{\prime}$, consequentemente $\Phi \in H\left(B_{s}(d), E\right)$.

Proposição 2.1.7 Sejam $E, F$ espaços de Banach de dimensão finita, $c \in E$ ed $\in F$. Se $A:\left(\mathcal{H}(c), \tau_{0}\right) \rightarrow\left(\mathcal{H}(d), \tau_{0}\right)$ é um homomorfismo local contínuo, então A é um operador de composição. 
Demonstração. Mostraremos inicialmente que $A\left(1^{c}\right)=1^{d}$. Seja $g \in H\left(B_{\frac{1}{m}}(d)\right)$ tal que $A\left(1^{c}\right)=[g]$, logo $[g][g]=A\left(1^{c}\right) A\left(1^{c}\right)=A\left(1^{c}\right)=[g]$ então existe $m_{1}>m$ tal que $g(x)(1-g(x))=0, \forall x \in B_{\frac{1}{m_{1}}}(d)$. Como $A \neq 0$, temos que $g \neq 0$, assim existe $m_{2}>m_{1}$ tal que $g(x)=1, \forall x \in B_{\frac{1}{m_{2}}}(d)$, consequentemente $[g]=1^{d}$. Portanto, $A\left(1^{c}\right)=1^{d}$.

Agora, mostraremos que para cada $n \in \mathbb{N}$, existe um homomorfismo contínuo $\psi_{n}:\left(H\left(B_{\frac{1}{n}}(c)\right), \tau_{0}\right) \rightarrow\left(H\left(B_{\frac{1}{m_{n}}}(d)\right), \tau_{0}\right)$ tal que $A \circ \mathcal{I}_{n}^{c}=\mathcal{I}_{m_{n}}^{d} \circ \psi_{n}$.

Como $A \circ \mathcal{I}_{n}^{c}: H\left(B_{\frac{1}{n}}(c)\right) \rightarrow\left(\mathcal{H}(d), \tau_{0}\right)$ é um homomorfismo contínuo, segue do teorema 1.2.11 de Grothendieck, que existem um conjunto aberto $B_{\frac{1}{m_{n}}}(d)$ e uma aplicação linear contínua

$$
\psi_{n}:\left(H\left(B_{\frac{1}{n}}(c)\right), \tau_{0}\right) \rightarrow\left(H\left(B_{\frac{1}{m_{n}}}(d)\right), \tau_{0}\right) \text { tal que } A \circ \mathcal{I}_{n}^{c}=\mathcal{I}_{m_{n}}^{d} \circ \psi_{n}
$$

Sem perda de generalidade podemos supor que $m_{1}<m_{2}<m_{3} \cdots<m_{n}<\cdots$.

Observamos que cada $\mathcal{I}_{n}^{c}$ e $\mathcal{I}_{m}^{d}$ são homomorfismos contínuos e como $A$ é um homomorfismo contínuo, por (2.4) temos:

$$
\left[\psi_{n}(f \cdot g)\right]=A\left(\mathcal{I}_{n}^{c}(f) \cdot \mathcal{I}_{n}^{c}(g)\right)=\left[\psi_{n}(f)\right]\left[\psi_{n}(g)\right]=\left[\psi_{n}(f) \cdot \psi_{n}(g)\right]
$$

isto é, $\psi_{n}(f \cdot g) \sim \psi_{n}(f) \cdot \psi_{n}(g), \forall f, g \in H\left(B_{\frac{1}{n}}(c)\right)$. Consequentemente, existe um conjunto aberto $W \subset B_{\frac{1}{m_{n}}}(d)$ tal que $\psi_{n}(f \cdot g)_{\left.\right|_{W}}=\psi_{n}(f) \cdot \psi_{n}(g)_{\left.\right|_{W}}$, como $B_{\frac{1}{m_{n}}}(d)$ é conexo temos que $\psi_{n}(f \cdot g)=\psi_{n}(f) \cdot \psi_{n}(g), \forall f, g \in H\left(B_{\frac{1}{n}}(c)\right)$. Assim, $\psi_{n}:\left(H\left(B_{\frac{1}{n}}(c)\right), \tau_{0}\right) \rightarrow$ $\left(H\left(B_{\frac{1}{m_{n}}}(d)\right), \tau_{0}\right)$ é um homomorfismo.

Pela proposição 2.1.6 temos que cada homomorfismo contínuo $\psi_{n}:\left(H\left(B_{\frac{1}{n}}(c)\right), \tau_{0}\right) \rightarrow$ $\left(H\left(B_{\frac{1}{m_{n}}}(d)\right), \tau_{0}\right)$ é um operador de composição, isto é, existe uma aplicação holomorfa $\Phi_{n}: B_{\frac{1}{m_{n}}}(d) \rightarrow E \operatorname{com} \Phi_{n}\left(B_{\frac{1}{m_{n}}}(d)\right) \subset B_{\frac{1}{n}}(c)$ tal que $\psi_{n}(f)=f \circ \Phi_{n}, \forall f \in H\left(B_{\frac{1}{n}}(c)\right)$.

Finalmente, mostraremos que $A:\left(\mathcal{H}(c), \tau_{0}\right) \rightarrow\left(\mathcal{H}(d), \tau_{0}\right)$ é um operador de composição. Isto é, mostraremos que existe uma aplicação holomorfa $\Phi: B_{\frac{1}{m_{1}}}(d) \rightarrow E$ tal que $\Phi(d)=c$ e $A([f])=[f \circ \Phi], \forall f \in H\left(B_{\frac{1}{n}}(c)\right), \forall n \in \mathbb{N}$.

Consideremos a aplicação holomorfa $\Phi=\Phi_{1}: B_{\frac{1}{m_{1}}}(d) \rightarrow E \operatorname{com} \Phi\left(B_{\frac{1}{m_{1}}}(d)\right) \subset B_{1}(c)$. Vamos mostrar que $\Phi_{\left.\right|_{B} \frac{1}{m_{n}}(d)}=\Phi_{n}, \forall n \in \mathbb{N}$. De fato, como $\left(E^{\prime},\|\|\right) \hookrightarrow\left(H\left(B_{\frac{1}{n}}(c)\right), \tau_{0}\right)$ e $\left(E^{\prime},\|\|\right) \hookrightarrow\left(H\left(B_{1}(c)\right), \tau_{0}\right)$ temos que $\left[x^{\prime} \circ \Phi_{1}\right]=A\left(\left[x^{\prime}\right]\right)=\left[x^{\prime} \circ \Phi_{n}\right], \forall x^{\prime} \in E^{\prime}$ 
isto é, $x^{\prime} \circ \Phi_{1} \sim x^{\prime} \circ \Phi_{n}, \forall x^{\prime} \in E^{\prime}$. Logo existe um conjunto aberto $W_{d}$ contendo $d$ tais que $W_{d} \subset B_{\frac{1}{m_{1}}}(d) \cap B_{\frac{1}{m_{n}}}(d)$ e $\left(x^{\prime} \circ \Phi_{1}\right)_{\left.\right|_{W_{d}}}=\left(x^{\prime} \circ \Phi_{n}\right)_{\left.\right|_{W_{d}}}, \forall x^{\prime} \in E^{\prime}$. Como $B_{\frac{1}{m_{n}}}(d)$ é conexo $\left(x^{\prime} \circ \Phi_{1}\right)(y)=\left(x^{\prime} \circ \Phi_{n}\right)(y), \forall y \in B_{\frac{1}{m_{n}}}(d), \forall x^{\prime} \in E^{\prime}$ e consequentemente pelo teorema de Hanh-Banach temos que $\Phi_{1}(y)=\Phi_{n}(y), \forall y \in B_{\frac{1}{m_{n}}}(d)$, isto é, $\Phi_{\left.\right|_{B} \frac{1}{m_{n}}(d)}=\Phi_{n}, \forall n \in \mathbb{N}$.

Vamos agora mostrar que $\Phi(d)=c$. Dado que $\Phi(d)=\Phi_{n}(d) \in B_{\frac{1}{m_{n}}}(d), \forall n \in \mathbb{N}$, temos $\Phi(d) \in B_{\frac{1}{n}}(c), \forall n \in \mathbb{N}$ e consequentemente $\Phi(d)=c$.

Portanto, $A([f])=[f \circ \Phi], \forall f \in H\left(B_{\frac{1}{n}}(c)\right), \forall n \in \mathbb{N}$.

Na seguinte proposição mostramos o caso de operadores de composição quando $E$ e $F$ são espaços de Banach de dimensão finita.

Proposição 2.1.8 Sejam $E, F$ espaços de Banach de dimensão finita, $K \subset E$ subconjunto compacto polinomialmente convexo e $L \subset F$ um subconjunto compacto. Se $A:\left(\mathcal{H}(K), \tau_{w}\right) \rightarrow\left(\mathcal{H}(L), \tau_{w}\right)$ é um homomorfismo contínuo então $A$ é um operador de composição.

Demonstração. Vamos considerar o limite indutivo $\left(\mathcal{H}(L), \tau_{w}\right)=\lim _{\overrightarrow{V \supset L}} H^{\infty}(V)$. Dado que a dimensão de $E^{\prime}$ é finita temos que a inclusão $\left(E^{\prime}, \tau_{0}\right) \hookrightarrow\left(\mathcal{H}(K), \tau_{w}\right)$ é contínua, $\operatorname{logo} A_{\left.\right|_{E^{\prime}}}:\left(E^{\prime}, \tau_{0}\right) \rightarrow\left(\mathcal{H}(L), \tau_{w}\right)$ é um homomorfismo contínuo. Pelo teorema 1.2.11 de Grothendieck, temos que existe um conjunto aberto $V \supset L$ e uma aplicação linear contínua $\psi:\left(E^{\prime}, \tau_{0}\right) \rightarrow\left(H(V), \tau_{w}\right)$ tal que

$$
\mathcal{I}_{V}^{L} \circ \psi=A_{I_{E^{\prime}}}
$$

Agora, vamos mostrar que existe uma aplicação holomorfa $\Phi: V \rightarrow E$ tal que $\psi(\ell)=\ell \circ \Phi, \forall \ell \in E^{\prime}$. De fato, para cada $y \in V$, consideramos o homomorfismo contínuo $\delta_{y}:\left(H(V), \tau_{w}\right) \rightarrow \mathbb{C}$. Logo $\delta_{y} \circ \psi:\left(E^{\prime}, \tau_{0}\right) \rightarrow \mathbb{C}$ é uma aplicação linear contínua e como $E^{\prime}$ tem dimensão finita $\left(\delta_{y} \circ \psi\right)_{\left.\right|_{E^{\prime}}} \in E^{\prime \prime}=E$, assim $\exists ! x(y) \in E$ tal que

$$
\delta_{y} \circ \psi(\ell)=\ell(x(y)), \forall \ell \in E^{\prime}
$$

Assim, definimos a aplicação $\Phi: V \rightarrow E$ por $\Phi(y)=x(y), \forall y \in V$ e consequentemente $\ell \circ \Phi=\psi(\ell)$. Como $\psi(\ell) \in H(V), \forall \ell \in E^{\prime}$, claramente $\Phi \in H(V, E)$. 
Afirmamos que $\Phi(L) \subset K$. De fato, seja $y \in L$ e $\rho_{y}:\left(\mathcal{H}(L), \tau_{w}\right) \rightarrow \mathbb{C}$ tal que $\rho_{y}([g])=g(y), \forall[g] \in \mathcal{H}(L)$ um homomorfismo contínuo. Logo $\rho_{y} \circ A:\left(\mathcal{H}(K), \tau_{w}\right) \rightarrow \mathbb{C}$ é um homomorfismo contínuo. Assim para cada conjunto aberto $U \supset K$ existe uma constante $c(U)>0$ tal que $\left|\rho_{y} \circ A(f)\right| \leq c(U)\|f\|_{U}$ para cada $f \in H^{\infty}(U)$.

Colocando $f^{n}$ no lugar de $f$, temos que $\left|\rho_{y} \circ A(f)\right| \leq \sqrt[n]{c(U)}\|f\|_{U}, \forall f \in H^{\infty}(U), \forall n \in$ $\mathbb{N}$. Fazendo $n \rightarrow \infty$, temos que $\left|\rho_{y} \circ A(f)\right| \leq\|f\|_{U}, \forall f \in H^{\infty}(U)$. Como $U \supset K$ é arbitrário temos que

$$
\left|\rho_{y} \circ A(f)\right| \leq\|f\|_{K}, \forall f \in \mathcal{H}(K)
$$

De (2.5) e (2.6) temos que $\rho_{y} \circ A(\ell)=\delta_{y} \circ \psi(\ell)=\ell(x(y)), \forall \ell \in E^{\prime}$. Como $\mathcal{P}_{f}(E)=\mathcal{P}(E)$, temos que $\rho_{y} \circ A(P)=P(x(y)), \forall P \in \mathcal{P}(E)$. Logo em (2.7) temos que $|P(x(y))| \leq\|P\|_{\left.\right|_{K}}, \forall P \in \mathcal{P}(E), \operatorname{assim} x(y) \in \widehat{K}_{\mathcal{P}(E)}$ e dado que $K$ é polinomialmente convexo, seque que $\Phi(y)=x(y) \in K$. Portanto $\Phi(L) \subset K$.

Finalmente, consideramos o homomorfismo contínuo $\Gamma:\left(\mathcal{H}(K), \tau_{w}\right) \rightarrow\left(\mathcal{H}(L), \tau_{w}\right)$ tal que $\Gamma([f])=[f \circ \Phi], \forall f \in \mathcal{H}(K)$. Consequentemente,

$$
\Gamma(\ell)=[\ell \circ \Phi]=[\psi(\ell)]=\mathcal{I}_{V}^{L} \circ \psi(\ell)=A(\ell), \forall \ell \in E^{\prime}
$$

além disso, $\Gamma$ e $A$ são homomorfismos e $\Gamma(P)=A(P), \forall P \in \mathcal{P}_{f}(E)$. Como $\left(\mathcal{P}_{f}(E), \tau_{w}\right)$ é denso em $\left(\mathcal{H}(K), \tau_{w}\right)$ temos $\Gamma(f)=A(f), \forall f \in \mathcal{H}(K)$. Assim $A([f])=[f \circ \Phi], \forall f \in$ $\mathcal{H}(K)$, e consequentemente $A$ é um operador de composição.

Por outro lado, O. Nicodemi em [28] observou que sob certas condições todo homomofismo $A:\left(\mathcal{H}(K), \tau_{0}\right) \rightarrow\left(\mathcal{H}(L), \tau_{0}\right)$ é operador de composição e mostrou por um exemplo que isto não é verdade em geral. Nicodemi estendeu qualquer aplicação linear $A$ entre duais de espaços de Banach, $A: E^{\prime} \rightarrow F^{\prime}$, para um homomorfismo $\widetilde{A}: \mathcal{H}(\{0\}) \rightarrow \mathcal{H}(\{0\})$ entre álgebras de germes holomorfos na origem e mostra que se $A$ não é a transposta de uma aplicação linear de $F$ em $E$, então $\widetilde{A}$ não será um operador de composição. Mais precisamente, ela considerou $E, F$ espaços de Banach, $V \subset F$ um conjunto aberto com $0 \in V,\{0\}_{E}$ e $\{0\}_{F}$ conjuntos formados pelo 0 de $E$ e $F$, e uma aplicação linear contínua $A: E^{\prime} \rightarrow H^{\infty}(V)$ tal que $A\left(x^{\prime}\right)(0)=0, \forall x^{\prime} \in E^{\prime}$. Por um processo indutivo ela construiu um homomorfismo contínuo $\widetilde{A}: \mathcal{H}\left(\{0\}_{E}\right) \rightarrow \mathcal{H}\left(\{0\}_{F}\right)$ onde $\mathcal{H}\left(\{0\}_{E}\right)$ está munido 
da topologia limite indutivo das funções holomorfas limitadas numa vizinhança de zero. Tal homomorfismo é uma extensão de $A$. Agora, ela mostrou que se $A$ é um operador de composição, então $\widetilde{A}$ também é um operador de composição e concluiu seu contra-exemplo observando que a aplicação inclusão natural $A: E^{\prime} \rightarrow E^{\prime \prime \prime}$ é a transposta de uma aplicação linear $f: E^{\prime \prime} \rightarrow E$ se, e somente se, $E$ é reflexivo. Assim $\widetilde{A}$ é operador de composição se, e somente se, $E$ é reflexivo.

\subsection{Homomorfismos contínuos entre certas álgebras de funções holomorfas}

Em [16] J. M. Isidro caracterizou o espectro de álgebras topológicas de funções holomorfas $(H(U), \tau)$ onde $\tau=\tau_{0}$ ou $\tau_{w}$ entre outras topologias. Usando os resultados de Isidro mostramos que sob certas condições homomorfismos contínuos entre estas álgebras topológicas são operadores de composição.

Proposição 2.2.1 Sejam E, F espaços localmente convexos complexos tal que E é Hausdorff, completo e tem a propriedade de aproximação. Sejam $U \subset E$ um conjunto aberto, convexo e equilibrado, $V \subset F$ um conjunto aberto e $A: H(U) \rightarrow H(V)$ um homomorfismo. As seguintes afirmações são equivalentes.

(a) $A:\left(H(U), \tau_{w}\right) \rightarrow\left(H(V), \tau_{w}\right)$ é contínuo.

(b) $A:\left(H(U), \tau_{w}\right) \rightarrow\left(H(V), \tau_{0}\right)$ é contínuo.

(c) A é um operador de composição.

\section{Demonstração.}

$(a) \Rightarrow(b)$. Seja $A:\left(H(U), \tau_{w}\right) \rightarrow\left(H(V), \tau_{w}\right)$ é um homomorfismo contínuo. Dado que a inclusão $\left(H(V), \tau_{w}\right) \hookrightarrow\left(H(V), \tau_{0}\right)$ é contínua, temos que $A:\left(H(U), \tau_{w}\right) \rightarrow\left(H(V), \tau_{0}\right)$ é um homomorfismo contínuo. 
$(b) \Rightarrow(c)$. Seja $A:\left(H(U), \tau_{w}\right) \rightarrow\left(H(V), \tau_{0}\right)$ um homomorfismo contínuo. Vamos mostrar que $A$ é um operador de composição.

Para cada $y \in V$, consideremos o homomorfismo contínuo $\delta_{y}:\left(H(V), \tau_{0}\right) \rightarrow \mathbb{C}$. Assim $\delta_{y} \circ A:\left(H(U), \tau_{w}\right) \rightarrow \mathbb{C}$ é um homomorfismo contínuo e pelo corolário 2 de [16] (ver, pg. 413), existe um único $x(y) \in U$ tal que $\delta_{y} \circ A(f)=f(x(y)), \forall f \in H(U), \forall y \in V$.

Logo, podemos definir uma aplicação $\Phi: V \rightarrow U$ dado por $\Phi(y)=x(y), \forall y \in V$ e consequentemente $A(f)=f \circ \Phi, \forall f \in H(U)$.

$(c) \Rightarrow(a)$. Seja $A:\left(H(U), \tau_{w}\right) \rightarrow\left(H(V), \tau_{w}\right)$ um operador de composição, isto é, existe uma aplicação holomorfa $\Phi: V \rightarrow U$ tal que $A(f)=f \circ \Phi, \forall f \in H(U)$.

Seja $q: H(V) \rightarrow \mathbb{R}$ uma seminorma em $H(V)$ portada pelo conjunto compacto $L$. Consideremos a aplicação $p: H(U) \rightarrow \mathbb{R}$ dada por $p(f)=q(A(f)), \forall f \in H(U)$. Como $A$ é linear temos que $p$ é uma seminorma em $H(U)$. Mostraremos que esta seminorma $p$ é portada pelo conjunto compacto $\Phi(L) \subset E$. Seja $U_{1} \subset U$ um conjunto aberto tal que $\Phi(L) \subset U_{1} \subset U$ e assim $L \subset \Phi^{-1}\left(U_{1}\right) \subset V$ onde $\Phi^{-1}\left(U_{1}\right)$ é um conjunto aberto.

Como $q$ portada por $L$ existe $C_{U_{1}}>0$ tal que $q(g) \leq C_{U_{1}}\|g\|_{\Phi^{-1}\left(U_{1}\right)}, \forall g \in H(V)$, $\operatorname{assim} p(f)=q(A(f)) \leq C_{U_{1}}\|f \circ \Phi\|_{\Phi^{-1}\left(U_{1}\right)} \leq C_{U_{1}}\|f\|_{U_{1}}, \forall f \in H(U)$. Segue, da proposição 2 pg. 97 de [15], que $A$ é contínua.

$\mathrm{Na}$ seguinte proposição 2.2 .2 mostramos que todo operador de composição entre germes holomorfos é contínuo na topologia de Nachbin. Este resultado será usado no capítulo 3 .

Proposição 2.2.2 Sejam E, $F$ espaços de Banach complexos, $K \subset E$ e $L \subset F$ subconjuntos compactos. Então cada operador de composição $A:\left(\mathcal{H}(K), \tau_{w}\right) \rightarrow\left(\mathcal{H}(L), \tau_{w}\right)$ é contínuo.

Demonstração. Seja $A:\left(\mathcal{H}(K), \tau_{w}\right) \rightarrow\left(\mathcal{H}(L), \tau_{w}\right)$ um operador de composição, isto é, existem um aberto $V_{0} \supset L$ e uma função holomorfa $\Phi: V_{0} \rightarrow E$ tais que $\Phi(L) \subset K$ e $A([f])=[f \circ \Phi]$ para cada função holomorfa $f$ definida sobre uma vizinhança de $K$. 
Assim, para cada conjunto aberto $U \supset K$ existem um conjunto aberto $V$ tal que $L \subset V \subset V_{0} \operatorname{com} \Phi(V) \subset U$ e um operador de composição $\widetilde{A}_{U}:\left(H(U), \tau_{w}\right) \rightarrow\left(H(V), \tau_{w}\right)$ dado por $\widetilde{A}_{U}(f)=f \circ \Phi, \forall f \in H(U)$. Portanto, $A \circ \mathcal{I}_{U}^{K}=\mathcal{I}_{V}^{L} \circ \widetilde{A}_{U}$. Isto é, o seguinte diagrama é comutativo.

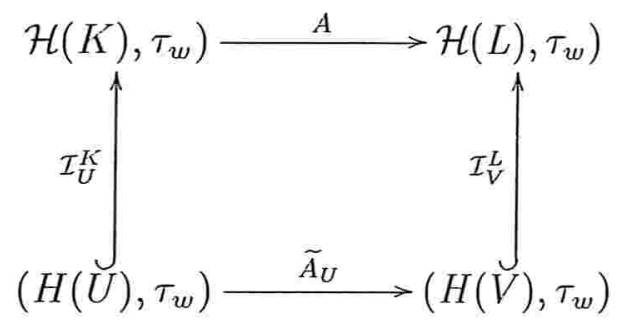

De forma análoga a demonstração $((c) \Rightarrow(a))$ da proposição 2.2 .1 temos que $\widetilde{A}_{U}$ é contínuo e pela propriedade dos limites indutivos (ver [27, pg. 104]) $A$ é contínuo.

Em [21] J. Mujica demonstrou que cada homomorfismo contínuo complexo em $\left(H(U), \tau_{w}\right)$, onde $U$ é um conjunto aberto polinomialmente convexo de um espaço de Fréchet com a propriedade de aproximação, é uma avaliação. Posteriormente, em [22] Mujica demonstrou tal resultado para abertos polinomialmente convexos em espaços localmente convexos quase-completos com a propriedade de aproximação. Estendendo assim o corolário 2 de J. M. Isidro [16]. Como consequência do referido resultado de Mujica podemos provar a seguinte proposição.

Proposição 2.2.3 Sejam E um espaço localmente convexo quase-completo com propriedade de aproximação, $F$ um espaço localmente convexo, $U \subset E$ um subconjunto aberto polinomialmente convexo e $V \subset F$ um conjunto aberto. Seja $A: H(U) \rightarrow H(V)$ um homomorfismo. As seguintes afirmações são equivalentes.

(a) $A:\left(H(U), \tau_{w}\right) \rightarrow\left(H(V), \tau_{w}\right)$ é contínuo.

(b) $A:\left(H(U), \tau_{w}\right) \rightarrow\left(H(V), \tau_{0}\right)$ é contínuo.

(c) A é um operador de composição. 
Demonstração. Omitiremos aqui tal demonstração, uma vez que é análogo a demonstração da proposição 2.2.1, fazendo-se as devidas mudanças.

Os resultados que apresentamos a seguir são válidos quando $E$ é um espaço de Tsirelson. Tsirelson em [32] descreveu um método de como construir uma classe de espaços com propriedades especiais. Não descreveremos aqui a construção de tais espaços, mas sim as propriedades que o espaço de Tsirelson $E$ possui:

(1) E é um espaço de Banach reflexivo de dimensão infinita, com base incondicional de Schauder, que não contém subespaços isomorfos a $c_{0}$ ou quaisquer $\ell^{p}(1 \leq p<\infty)$. Ver a construção em [32].

(2) $\mathcal{P}\left({ }^{n} E\right)$ é reflexivo para cada $n \in \mathbb{N}$.

Ver a demonstração de Alencar, Aron e Dineen em [1].

(3) $H_{b}(U)$ é reflexivo para um subconjunto aberto equilibrado $U \subset E$.

Ver a demonstração de Ansemil e Ponte em [2].

Vamos agora apresentar a demonstração de que todo homomorfismo contínuo $T: H_{b}(U) \rightarrow H_{b}(V)$ é um operador de composição sempre que $E$ seja um espaço de Tsirelson, $U \subset E$ um subconjunto aberto absolutamente convexo e $V \subset F$ é um subconjunto aberto de um espaço de Banach $F$. Tal resultado é uma consequência direta da proposição 3 de [13], como observaram os autores imediatamente após a demonstração da mesma. Este resultado foi também provado, independentemente, por Mujica em [23].

A demonstração que incluimos aqui do corolário 2.2.7 é a observada em [13]. Como os resultados descritos no lema 2.2 .4 e na proposição 2.2.6, foram comentados em [13], mas não foram incluidas suas demonstrações, decidimos por incluí-las aqui.

O corolário 2.2 .7 será usado para mostrar que homomorfismos entre álgebras de germes de funções holomorfas definidas em compactos de um espaço de Tsirelson são operadores de composição. 
Lema 2.2.4 Sejam $E$ um espaço de Tsirelson, $U \subset E$ um subconjunto aberto e $\mathcal{X} \subset$ $H_{b}(U)$ um conjunto limitado, então $\mathcal{X}$ é $\tau_{0}$-relativamente compacto em $H_{b}(U)$.

Demonstração. Seja $\mathcal{X} \subset H_{b}(U)$ limitado. Como os conjuntos compactos de $U$ são $U$-limitados, temos que $\mathcal{X}$ é $\tau_{0}$-limitado. Agora, segue da proposição 9.16 de [18] que $\mathcal{X}$ é $\tau_{0}$-relativamente compacto em $H(U)$. Assim, resta mostrar que $\overline{\mathcal{X}}^{\tau_{0}} \subset H_{b}(U)$. Seja $f \in \overline{\mathcal{X}}^{\tau_{0}}$, então existe uma rede $\left(f_{\alpha}\right)_{\alpha} \subset \mathcal{X}$ tal que $f_{\alpha} \stackrel{\tau_{0}}{\rightarrow} f$.

Como $E$ é um espaço de Tsirelson, temos que $H_{b}(U)$ é reflexivo, e consequentemente $\mathcal{X}$ é relativamente fracamente compacto em $H_{b}(U)$. Assim, existe uma subrede $\left(f_{\beta}\right)_{\beta} \subset \mathcal{X}$ de $\left(f_{\alpha}\right)_{\alpha}$ que converge fracamente em $H_{b}(U)$. Isto é, $\exists g \in H_{b}(U)$ tal que $f_{\beta} \stackrel{w}{\rightarrow} g$. Como para cada $x \in U$, temos que $\delta_{x} \in H_{b}(U)^{\prime}$, segue que $f_{\beta}(x) \rightarrow g(x)$.

Agora, como $f_{\beta} \stackrel{\tau_{0}}{\rightarrow} f$, temos que $f_{\beta}(x) \rightarrow f(x)$ para cada $x \in U$. Da unicidade do limite, temos que $f(x)=g(x)$ para cada $x \in U$. Consequentemente $f=g \in H_{b}(U)$.

Para demonstrar a proposição 2.2.6 utilizaremos o seguinte lema.

Lema 2.2.5 Sejam E um espaço de Tsirelson e U um subconjunto aberto de E. Então

(a) A inclusão $\iota:\left(H_{b}(U), w\right) \hookrightarrow\left(H_{b}(U), \tau_{p}\right)$ é um homeomorfismo sobre os limitados de $H_{b}(U)$, onde $w$ é a topologia fraca e $\tau_{p}$ é a topologia pontual.

(b) A inclusão $\iota:\left(H_{b}(U), \tau_{0}\right) \hookrightarrow\left(H_{b}(U), \tau_{p}\right)$ é um homeomorfismo sobre os limitados de $H_{b}(U)$.

Demonstração. $\quad(a)$ A inclusão $\iota:\left(H_{b}(U), w\right) \hookrightarrow\left(H_{b}(U), \tau_{p}\right)$ é contínua. Seja $\mathcal{X} \subset$ $H_{b}(U)$ limitado. Como $H_{b}(U)$ é reflexivo, $\mathcal{X}$ é relativamente fracamente compacto. Assim, $\iota_{x}$ é um homeomorfismo.

(b) A inclusão $\iota:\left(H_{b}(U), \tau_{0}\right) \hookrightarrow\left(H_{b}(U), \tau_{p}\right)$ é contínua. Para cada $\mathcal{X} \subset H_{b}(U)$ limitado temos pelo lema 2.2 .4 que $\mathcal{X}$ é $\tau_{0}$-relativamente compacto em $H_{b}(U)$. Consequentemente, $\iota_{\chi}$ é um homeomorfismo. 
Proposição 2.2.6 Sejam $E$ um espaço de Tsirelson, $F$ um espaço de Banach, $U \subset E$ um subconjunto aberto absolutamente convexo e $V \subset F$ um aberto. Seja $T: H_{b}(U) \rightarrow H_{b}(V)$ um homomorfismo contínuo, então $T$ é $\tau_{0}$-pontualmente contínuo sobre os limitados de $H_{b}(U)$.

Demonstração. Seja $T: H_{b}(U) \rightarrow H_{b}(V)$ um homomorfismo contínuo. Vamos mostrar que para cada $y \in V$, a aplicação $\delta_{y} \circ T: H_{b}(U) \rightarrow \mathbb{C}$ é $\tau_{0}$-contínua sobre subconjuntos limitados de $H_{b}(U)$.

Como $\delta_{y} \circ T \in H_{b}(U)^{\prime}$, segue que $\delta_{y} \circ T$ é fracamente contínua e assim $\left(\delta_{y} \circ T\right)_{\left.\right|_{\mathcal{X}}}$ é fracamente contínua para cada $\mathcal{X} \subset H_{b}(U)$ limitado. Usando o lema 2.2 .5 itens $(a)$ e $(b)$ temos que $\left(\delta_{y} \circ T\right)_{\left.\right|_{\chi}}$ é $\tau_{0}$-contínuo. Isto é, $T$ é $\tau_{0}$-pontualmente contínuo sobre os limitados de $H_{b}(U)$.

Corolário 2.2.7 Sejam E um espaço de Tsirelson, $F$ um espaço de Banach, $U \subset E$ um conjunto aberto absolutamente convexo e $V \subset F$ um aberto. Seja $T: H_{b}(U) \rightarrow H_{b}(V)$ um homomorfismo contínuo, então $T$ é um operador de composição.

Demonstração. Seja $T: H_{b}(U) \rightarrow H_{b}(V)$ um homomorfismo contínuo. Da proposição 2.2.6 segue que $T: H_{b}(U) \rightarrow H_{b}(V)$ é $\tau_{0}$-pontualmente contínua sobre os conjuntos limitados de $H_{b}(U)$ e da proposição 3 de [13] concluimos que $T$ é um operador de composição. 


\section{Capítulo 3}

\section{Homomorfismos contínuos entre álgebras de germes holomorfos}

Neste capítulo estudamos homomorfismos contínuos entre álgebras de germes de funções holomorfas munidas da topologia de Nachbin. Apresentamos uma caracterização para que tais homomorfismos sejam operadores de composição. A partir daí, passamos a estudar a compacidade e a compacidade fraca de operadores de composição entre álgebras de germes de funções holomorfas.

No decorrer deste capítulo estaremos usando as notações introduzidas em 1.2.5.

\subsection{Homomorfismos contínuos entre álgebras de ger- mes holomorfos}

Nesta seção apresentamos uma caracterização de quando os homomorfismos contínuos entre álgebras de germes de funções holomorfas é um operador de composição.

A demonstração de tal resultado será feita utilizando uma fatoração através de álgebras de funções holomorfas do tipo limitado definidas em abertos de um espaço de 
Banach. Isto é o que faremos na proposição 3.1.1.

Proposição 3.1.1 Sejam E, $F$ espaços de Banach, $K \subset E$ e $L \subset F$ subconjuntos compactos. Seja $A:\left(\mathcal{H}(K), \tau_{w}\right) \rightarrow\left(\mathcal{H}(L), \tau_{w}\right)$ um homomorfismo contínuo. Então para cada aberto $U_{n} \supset K$ existem um aberto $V_{m_{n}} \supset L$ e um homomorfismo contínuo $\widetilde{A}_{n}: H_{b}\left(U_{n}\right) \rightarrow H_{b}\left(V_{m_{n}}\right)$ tal que $\mathcal{I}_{m_{n}}^{L} \circ \widetilde{A}_{n}=A \circ \mathcal{I}_{n}^{K}$.

Demonstração. Seja $U_{n} \supset K$ um conjunto aberto e consideremos o homomorfismo contínuo $A \circ \mathcal{I}_{n}^{K}: H_{b}\left(U_{n}\right) \rightarrow \mathcal{H}(L)$. Segue do teorema 1.2.11 de Grothendieck, que existem um conjunto aberto $V_{m_{l_{n}}} \supset L$ e uma aplicação linear contínua

$$
\overline{A \circ \mathcal{I}_{n}^{K}}: H_{b}\left(U_{n}\right) \rightarrow H_{b}\left(V_{m_{l_{n}}}\right) \text { tal que } \mathcal{I}_{m_{l_{n}}}^{L} \circ \overline{A \circ \mathcal{I}_{n}^{K}}=A \circ \mathcal{I}_{n}^{K} .
$$

Isto é, o seguinte diagrama é comutativo.

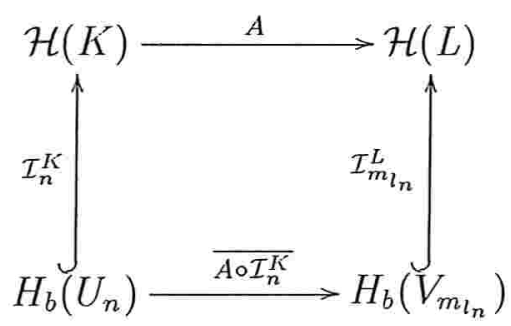

Observamos que cada $\mathcal{I}_{n}^{K}$ e $\mathcal{I}_{m}^{L}$ são homomorfismos contínuos, e como $A$ é um homomorfismo por (3.1) temos:

$$
\left[\overline{A \circ \mathcal{I}_{n}^{K}}(f \cdot g)\right]=A\left(\mathcal{I}_{n}^{K}(f) \cdot \mathcal{I}_{n}^{K}(g)\right)=\left[\overline{A \circ \mathcal{I}_{n}^{K}}(f)\right]\left[\overline{A \circ \mathcal{I}_{n}^{K}}(g)\right]=\left[\overline{A \circ \mathcal{I}_{n}^{K}}(f) \cdot \overline{A \circ \mathcal{I}_{n}^{K}}(g)\right]
$$

então, $\overline{A \circ \mathcal{I}_{n}^{K}}(f \cdot g) \sim \overline{A \circ \mathcal{I}_{n}^{K}}(f) \cdot \overline{A \circ \mathcal{I}_{n}^{K}}(g), \forall f, g \in H_{b}\left(U_{n}\right)$. Consequentemente, existe um conjunto aberto $V_{m_{n}} \subset V_{m_{l_{n}}}$ tal que $\overline{A \circ \mathcal{I}_{n}^{K}}(f \cdot g)_{\left.\right|_{V_{m_{n}}}}=\left(\overline{A \circ \mathcal{I}_{n}^{K}}(f) \cdot \overline{A \circ \mathcal{I}_{n}^{K}}(g)\right)_{\left.\right|_{V_{m_{n}}}}$ e $H_{b}\left(V_{m_{l_{n}}}\right) \stackrel{i_{m_{l_{n}}}^{L}}{\longleftrightarrow} H_{b}\left(V_{m_{n}}\right)$. Assim $\mathcal{I}_{m_{n}}^{L} \circ i_{m_{l_{n}}}^{L}=\mathcal{I}_{m_{l_{n}}}^{L} \quad \mathrm{e}$

$$
\left(i_{m_{l_{n}}}^{L} \circ \overline{A \circ \mathcal{I}_{n}^{K}}\right)(f \cdot g)=\left(i_{m_{l_{n}}}^{L} \circ \overline{A \circ \mathcal{I}_{n}^{K}}\right)(f) \cdot\left(i_{m_{l_{n}}}^{L} \circ \overline{A \circ \mathcal{I}_{n}^{K}}\right)(g)
$$

logo, definimos um homomorfismo contínuo $\widetilde{A}_{n}: H_{b}\left(U_{n}\right) \rightarrow H_{b}\left(V_{m_{n}}\right)$ por $\tilde{A}_{n}=i_{m_{l_{n}}}^{L} \circ \overline{A \circ \mathcal{I}_{n}^{K}}$ tal que $A \circ \mathcal{I}_{n}^{K}=\mathcal{I}_{m_{l_{n}}}^{L} \circ \overline{A \circ \mathcal{I}_{n}^{K}}=\mathcal{I}_{m_{n}}^{L} \circ i_{m_{l_{n}}}^{L} \circ \overline{A \circ \mathcal{I}_{n}^{K}}=\mathcal{I}_{m_{n}}^{L} \circ \tilde{A}_{n}$. 
Portanto, para cada aberto $U_{n} \supset K$ existem um aberto $V_{m_{n}} \supset L$ e um homomorfismo contínuo $\widetilde{A}_{n}: H_{b}\left(U_{n}\right) \rightarrow H_{b}\left(V_{m_{n}}\right)$ tal que o seguinte diagrama é comutativo.

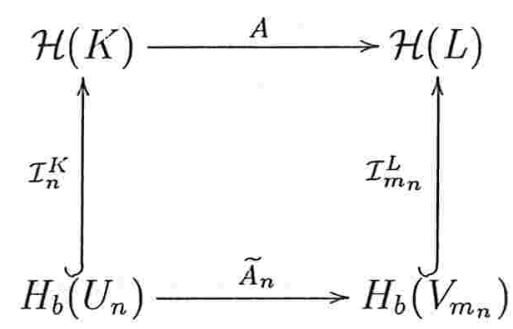

Isto é, $\mathcal{I}_{m_{n}}^{L} \circ \widetilde{A}_{n}=A \circ \mathcal{I}_{n}^{K}$.

Para demonstrar o principal resultado desta seção, vamos precisar do lema 3.1.2 a respeito de conjuntos $U$-limitados em um aberto $U$ de um espaço de Banach.

Lema 3.1.2 Sejam $E$ um espaço de Banach, $U$ e $W$ subconjuntos abertos de E. Seja $B \subset U \subset W$, tal que $B$ é $U$-limitado, então $B$ é $W$-limitado.

Demonstração. $\quad$ Seja $B \subset U \subset W, U$-limitado. Como, $d(B, \partial W) \geq d(B, \mathcal{C} W) \geq$ $d(B, \mathcal{C} U)$ basta mostrar que $d(B, \partial U)=d(B, \mathcal{C} U)$ para termos $d(B, \partial W)>0$, mostrando assim que $B$ é $W$-limitado.

Como $d(B, \partial U) \geq d(B, \mathcal{C} U)$, vamos mostrar que não pode ocorrer $d(B, \partial U)>$ $d(B, \mathcal{C} U)$. Caso ocorra, como $d(B, \mathcal{C} U)=\inf \{\|b-x\|: b \in B, x \in \mathcal{C} U\}$, da definição de ínfimo temos que existem $b \in B, x \in \mathcal{C} U$ tal que

$$
\|b-x\|<d(B, \partial U) \leq\left\|b^{\prime}-y\right\|, \forall b^{\prime} \in B, \forall y \in \partial U
$$

Temos dois casos:

(a) Se $x \in \partial U$ e por (3.2), teríamos que $\|b-x\|<\|b-x\|$ ( absurdo! ).

(b) Se $x \in \stackrel{\circ}{\stackrel{\mathcal{C}}{U}}$, o segmento $[b, x] \cap \partial U \neq \emptyset$; seja $c \in[b, x] \cap \partial U \operatorname{logo} c=\lambda b+(1-\lambda) x$, onde $0<\lambda<1$, assim $\|b-c\|=|1-\lambda|\|b-x\|$, analogamente $\|x-c\|=|\lambda|\|x-b\|$, então $\|b-c\|+\|x-c\|=\|x-b\|$ e por (3.2) temos $\|b-x\|<\|b-c\|$ daí $\|b-c\|+\|x-c\|<\|b-c\|$ logo $\|x-c\|<0$ ( absurdo! ). Portanto, $d(B, \partial U)=d(B, \mathcal{C} U)$. 
Assim $d(B, \partial W)>0$ e consequentemente $B$ é $W$-limitado.

Teorema 3.1.3 Sejam E, $F$ espaços de Banach, $K \subset E$ e $L \subset F$ subconjuntos compactos. Seja $A:\left(\mathcal{H}(K), \tau_{w}\right) \rightarrow\left(\mathcal{H}(L), \tau_{w}\right)$ um homomorfismo contínuo. Então $A$ é um operador de composição se, e somente se, para cada aberto $U_{n} \supset K$ existem um aberto $V_{m_{n}} \supset L$ e um operador de composição $\tilde{A}_{n}: H_{b}\left(U_{n}\right) \rightarrow H_{b}\left(V_{m_{n}}\right)$ tal que $\mathcal{I}_{m_{n}}^{L} \circ \widetilde{A}_{n}=A \circ \mathcal{I}_{n}^{K}$.

Demonstração. Usaremos as notações de 1.2 .5 para os limites indutivos $\left(\mathcal{H}(K), \tau_{w}\right)=$ $\lim _{\vec{n} \in \mathbb{\mathbb { N }}} H_{b}\left(U_{n}\right)$ e $\left(\mathcal{H}(L), \tau_{w}\right)=\underset{\overrightarrow{m \in \mathbb{N}}}{\lim _{\overrightarrow{\mathbb{N}}}} H_{b}\left(V_{m}\right)$.

Vamos primeiramente supor que para cada aberto $U_{n} \supset K$ existem um aberto $V_{m_{n}} \supset$ $L$ e $\widetilde{A}_{n}: H_{b}\left(U_{n}\right) \rightarrow H_{b}\left(V_{m_{n}}\right)$ um operador de composição tal que $\mathcal{I}_{m_{n}}^{L} \circ \widetilde{A}_{n}=A \circ \mathcal{I}_{n}^{K}$.

Sem perda de generalidade podemos supor que $V_{m_{1}} \supset V_{m_{2}} \supset V_{m_{3}} \supset \cdots$

Vamos demonstrar que $A$ é um operador de composição.

Como cada $\widetilde{A}_{n}: H_{b}\left(U_{n}\right) \rightarrow H_{b}\left(V_{m_{n}}\right)$ é um operador de composição, segue que existe uma aplicação $\Phi_{n} \in H_{b}\left(V_{m_{n}}, U_{n}\right)$ tal que $\widetilde{A}_{n}(f)=f \circ \Phi_{n}, \forall f \in H_{b}\left(U_{n}\right)$. Assim,

$$
A([f])=\left(A \circ \mathcal{I}_{n}^{K}\right)(f)=\left(\mathcal{I}_{m_{n}}^{L} \circ \widetilde{A}_{n}\right)(f)=\left[\widetilde{A}_{n}(f)\right]=\left[f \circ \Phi_{n}\right], \forall f \in H_{b}\left(U_{n}\right),
$$

para cada $n \in \mathbb{N}$.

Agora, afirmamos que existem um conjunto aberto $V_{m_{1}} \supset L$ e $\Phi \in H_{b}\left(V_{m_{1}}, E\right)$ tal que $\Phi(L) \subset K$, onde $A([f])=[f \circ \Phi], \forall f \in H_{b}\left(U_{n}\right)$, e para cada $n \in \mathbb{N}$.

De fato, considerando o conjunto $V_{m_{1}}$ acima podemos definir uma aplicação $\Phi: V_{m_{1}} \rightarrow E$ por $\Phi(y)=\Phi_{1}(y), \forall y \in V_{m_{1}}$.

Mostraremos que $\Phi=\Phi_{n}$ sobre cada $V_{m_{n}}$. Dado que $\left(E^{\prime},\|\|\right) \hookrightarrow H_{b}\left(U_{n}\right), \forall n \in \mathbb{N}$, segue de (3.3) que

$$
\left\{\begin{array}{l}
A\left(\left[x^{\prime}\right]\right)=\left[x^{\prime} \circ \Phi_{1}\right], \forall x^{\prime} \in E^{\prime} \quad e \\
A\left(\left[x^{\prime}\right]\right)=\left[x^{\prime} \circ \Phi_{n}\right], \forall x^{\prime} \in E^{\prime}, \forall n \in \mathbb{N}
\end{array}\right.
$$

consequentemente, $\left[x^{\prime} \circ \Phi_{1}\right]=\left[x^{\prime} \circ \Phi_{n}\right], \forall x^{\prime} \in E^{\prime}, \forall n \in \mathbb{N}$. Ou seja $x^{\prime} \circ \Phi_{1} \sim x^{\prime} \circ \Phi_{n}$, $\forall x^{\prime} \in E^{\prime}, \forall n \in \mathbb{N}$. Assim, existe um conjunto aberto $W \operatorname{com} L \subset W \subset V_{m_{n}}$ tal que $\left(x^{\prime} \circ \Phi_{1}\right)_{\left.\right|_{W}}=\left(x^{\prime} \circ \Phi_{n}\right)_{\left.\right|_{W}}, \forall x^{\prime} \in E^{\prime}$. 
Agora, seja $y \in V_{m_{n}}$ então $\exists z \in L$ tal que $y \in B_{\frac{1}{m_{n}}}(z)$. Como $L \subset W$ temos que $W \cap B_{\frac{1}{m_{n}}}(z) \neq \emptyset$ e consequentemente $\left(x^{\prime} \circ \Phi_{1}\right)_{\left.\right|_{W \cap B} \frac{1}{m_{n}}(z)}=\left(x^{\prime} \circ \Phi_{n}\right)_{\left.\right|_{W \cap B} \frac{1}{m_{n}}(z)}, \forall x^{\prime} \in E^{\prime}$. Mas $B_{\frac{1}{m_{n}}}(z)$ é conexo, logo $\left(x^{\prime} \circ \Phi_{1}\right)_{\left.\right|_{B_{\frac{1}{m_{n}}}}(z)}=\left(x^{\prime} \circ \Phi_{n}\right)_{\left.\right|_{B_{\frac{1}{m_{n}}}}(z)}, \forall x^{\prime} \in E^{\prime}$.

Assim, $\left(x^{\prime} \circ \Phi_{1}\right)(y)=\left(x^{\prime} \circ \Phi_{n}\right)(y), \forall y \in V_{m_{n}}, \forall x^{\prime} \in E^{\prime}$. Usando o teorema de Hahn-Banach, segue que

$$
\Phi(y)=\Phi_{1}(y)=\Phi_{n}(y), \forall y \in V_{m_{n}}
$$

Portanto, $\Phi_{V_{m_{n}}}=\Phi_{n}$ para cada $n \in \mathbb{N}$ e claramente $\Phi \in H_{b}\left(V_{m_{1}}, E\right)$.

Vamos agora mostrar que $\Phi(L) \subset K$. Da equação (3.5) temos que $\Phi(L) \subset U_{n}, \forall n \in$ IN. Assim para cada $y \in L, \exists \xi_{n} \in K$ tal que $\left\|\Phi(y)-\xi_{n}\right\|<\frac{1}{n}$, para cada $n \in \mathbb{N}$, isto é, $d(\Phi(y), K)<\frac{1}{n}, \forall n \in \mathbb{N}, \forall y \in L$; fazendo $n \rightarrow \infty$, temos $d(\Phi(y), K)=0$, para cada $y \in L$ e consequentemente $\Phi(L) \subset K$. Isto demonstra que $A([f])=[f \circ \Phi], \forall f \in$ $H_{b}\left(U_{n}\right), \forall n \in \mathbb{N}$, ou seja, $A=A_{\Phi}$.

Reciprocamente, suponhamos que $A$ é um operador de composição. Vamos provar que para cada aberto $U_{n} \supset K$ existem um aberto $V_{m_{n}} \supset L$ e um operador de composição $\widetilde{A}_{n}: H_{b}\left(U_{n}\right) \rightarrow H_{b}\left(V_{m_{n}}\right)$ tal que $\mathcal{I}_{m_{n}}^{L} \circ \widetilde{A}_{n}=A \circ \mathcal{I}_{n}^{K}$.

Seja $U_{n} \supset K$ um conjunto aberto, pela proposição 3.1.1 temos que existem um aberto $V_{m_{l_{n}}} \supset L$ e um homomorfismo contínuo $\varphi_{n}: H_{b}\left(U_{n}\right) \rightarrow H_{b}\left(V_{m_{l_{n}}}\right)$ tal que $\mathcal{I}_{m_{l_{n}}}^{L} \circ \varphi_{n}=$ $A \circ \mathcal{I}_{n}^{K}$.

Dado que $A$ é um operador de composição, existem um conjunto aberto $V_{m_{0}} \supset L$, e $\Phi \in H_{b}\left(V_{m_{0}}, E\right) \operatorname{com} \Phi\left(V_{m_{0}}\right) \subset U_{n}$ tal que

$$
A([f])=[f \circ \Phi], \forall f \in H_{b}\left(U_{n}\right) \text { e } \Phi(L) \subset K .
$$

Seja $m_{k_{n}} \geq \max \left\{m_{l_{n}}, m_{0}\right\}$, então $V_{m_{k_{n}}} \subset V_{m_{l_{n}}} \cap V_{m_{0}}$ e temos a aplicação inclusão $H_{b}\left(V_{m_{l_{n}}}\right) \stackrel{i_{m}^{L}}{\hookrightarrow} H_{b}\left(V_{m_{k_{n}}}\right)$ com $\mathcal{I}_{m_{k_{n}}}^{L} \circ i_{m_{l_{n}}}^{L}=\mathcal{I}_{m_{l_{n}}}^{L}$. Assim definimos um homomorfismo contínuo $\psi_{n}: H_{b}\left(U_{n}\right) \rightarrow H_{b}\left(V_{m_{k_{n}}}\right)$ por

$$
\psi_{n}(f)=i_{m_{l_{n}}}^{L} \circ \varphi_{n}(f), \forall f \in H_{b}\left(U_{n}\right)
$$

e consequentemente $\mathcal{I}_{m_{k_{n}}}^{L} \circ \psi_{n}=A \circ \mathcal{I}_{n}^{K}$. 
De (3.6) e (3.7) temos que $[f \circ \Phi]=\left[\psi_{n}(f)\right], \forall f \in H_{b}\left(U_{n}\right)$ e portanto $f \circ \Phi \sim$ $\psi_{n}(f), \forall f \in H_{b}\left(U_{n}\right)$. Assim existe $V_{m_{n}} \subset V_{m_{k_{n}}}$ tal que $\psi_{n}(f)_{\left.\right|_{V_{m_{n}}}}=(f \circ \Phi)_{\left.\right|_{V_{m_{n}}}}$. Mas, $H_{b}\left(V_{m_{k_{n}}}\right) \stackrel{i_{m_{k_{n}}}^{L}}{\longrightarrow} H_{b}\left(V_{m_{n}}\right)$ e $\mathcal{I}_{m_{n}}^{L} \circ i_{m_{k_{n}}}^{L}=\mathcal{I}_{m_{k_{n}}}^{L}, \log \circ\left(i_{m_{k_{n}}}^{L} \circ \psi_{n}\right)(f)=(f \circ \Phi)_{\left.\right|_{V_{m_{n}}}}$.

Agora, definimos $\widetilde{A}_{n}: H_{b}\left(U_{n}\right) \rightarrow H_{b}\left(V_{m_{n}}\right)$ por $\widetilde{A}_{n}=i_{m_{k_{n}}}^{L} \circ \psi_{n}$. Assim $\widetilde{A}_{n}$ é um homomorfismo contínuo tal que $A \circ \mathcal{I}_{n}^{K}=\mathcal{I}_{m_{k_{n}}}^{L} \circ \psi_{n}=\mathcal{I}_{m_{n}}^{L} \circ i_{m_{k_{n}}}^{L} \circ \psi_{n}=\mathcal{I}_{m_{n}}^{L} \circ \widetilde{A}_{n} \mathrm{e}$ $\widetilde{A}_{n}(f)=(f \circ \Phi)_{V_{V_{m_{n}}}}$.

Além disso, podemos definir uma aplicação $\Phi_{n}: V_{m_{n}} \rightarrow E$ por $\Phi_{n}=\Phi_{\left.\right|_{V_{m_{n}}}}$ onde $\Phi_{n}\left(V_{m_{n}}\right) \subset U_{n}$. Desta forma $\widetilde{A}_{n}(f)=f \circ \Phi_{n} \quad$ e $\quad \Phi_{n} \in H\left(V_{m_{n}}, E\right)$.

Vamos agora mostrar que $\Phi_{n} \in H_{b}\left(V_{m_{n}}, E\right)$. Seja $B \subset V_{m_{n}} \subset V_{m_{0}}$ um conjunto $V_{m_{n}{ }^{-}}$ limitado. Do lema 3.1.2, temos que $B \subset V_{m_{0}}$ é $V_{m_{0}}$-limitado. Como $\Phi \in H_{b}\left(V_{m_{0}}, E\right)$ temos que $\Phi(B) \subset E$ é limitado e portanto $\Phi_{n}(B) \subset E$ é limitado, isto é, $\Phi_{n} \in H_{b}\left(V_{m_{n}}, E\right)$.

Assim, para cada aberto $U_{n} \supset K$ existem um conjunto aberto $V_{m_{n}} \supset L$ e um operador de composição $\widetilde{A}_{n}: H_{b}\left(U_{n}\right) \rightarrow H_{b}\left(V_{m_{n}}\right)$ tal que $A \circ \mathcal{I}_{n}^{K}=\mathcal{I}_{m_{n}}^{L} \circ \widetilde{A}_{n}$.

O seguinte corolário dá uma condição necessária para que um homomorfismo contínuo entre germes seja um operador de composição.

Corolário 3.1.4 Sejam $E$ um espaço de Tsirelson e $F$ um espaço de Banach, $K \subset E$ compacto, convexo e equilibrado e $L \subset F$ um conjunto compacto. Então cada homomorfismo contínuo $A:\left(\mathcal{H}(K), \tau_{w}\right) \rightarrow\left(\mathcal{H}(L), \tau_{w}\right)$ é um operador de composição.

Demonstração. Seja $K \subset E$ um conjunto compacto, convexo e equilibrado. Então para cada $U_{n}=K+B_{\frac{1}{n}}(0) \subset E$, temos que $U_{n}$ é aberto convexo e equilibrado. Segue da proposição 3.1.1, que existem $V_{m_{n}} \supset L$ aberto e $\widetilde{A}_{n}: H_{b}\left(U_{n}\right) \rightarrow H_{b}\left(V_{m_{n}}\right)$ homomorfismo contínuo tal que $\mathcal{I}_{m_{n}}^{L} \circ \widetilde{A}_{n}=A \circ \mathcal{I}_{n}^{K}$.

Do corolario 2.2 .7 temos que $\widetilde{A}_{n}: H_{b}\left(U_{n}\right) \rightarrow H_{b}\left(V_{m_{n}}\right)$ é um operador de composição, isto é, existe uma única aplicação $\Phi_{n} \in H_{b}\left(V_{m_{n}}, U_{n}\right)$, tal que $\widetilde{A}_{n}(f)=f \circ \Phi_{n}, \forall f \in H_{b}\left(U_{n}\right)$.

Agora o resultado segue diretamente do teorema 3.1.3. 
Corolário 3.1.5 Seja E um espaço de Banach reflexivo tal que $\mathcal{P}_{f}\left({ }^{n} E\right)$ é denso em $\mathcal{P}\left({ }^{n} E\right)$ para cada $n \in \mathbb{N}$. Sejam $F$ um espaço de Banach, $K \subset E$ compacto, convexo e equilibrado e $L \subset F$ um conjunto compacto. Então cada homomorfismo contínuo $A:\left(\mathcal{H}(K), \tau_{w}\right) \rightarrow$ $\left(\mathcal{H}(L), \tau_{w}\right)$ é um operador de composição.

Demonstração. Segue do teorema 3.1.3 e do teorema 1.6 de J. Mujica [23].

\subsection{Homomorfismos contínuos entre as álgebras $H(U)$ e $\mathcal{H}(K)$}

Nesta seção daremos condições necessárias para que homomorfismos contínuos entre as álgebras $H(U)$ e $\mathcal{H}(K)$ sejam operadores de composição.

Definição 3.2.1 Sejam $E, F$ espaços de Banach complexos, $K \subset E, L \subset F$ subconjuntos compactos e $U \subset E, V \subset F$ subconjuntos abertos.

(a) Um homomorfismo $A: H_{b}(U) \rightarrow\left(\mathcal{H}(L), \tau_{w}\right)$ é um operador de composição, se existem um conjunto aberto $V \supset L$ e uma aplicação $\Phi \in H_{b}(V, E)$ tal que $\Phi(L) \subset U$ e $A(f)=[f \circ \Phi]$ para cada função holomorfa $f$ definida em $U$. Denotamos o operador de composição $A$ por $A_{\Phi}$.

(b) Um homomorfismo $A:\left(\mathcal{H}(K), \tau_{w}\right) \rightarrow H_{b}(V)$ é um operador de composição, se existe uma aplicação $\Phi \in H_{b}(V, E)$ tal que $\Phi(V) \subset K$ e $A([f])=f \circ \Phi$ para cada função holomorfa $f$ definida sobre uma vizinhança de $K$. Denotamos o operador de composição $A$ por $A_{\Phi}$.

Proposição 3.2.2 Sejam E um espaço de Tsirelson, $U \subset E$ um conjunto aberto convexo e equilibrado. Sejam F um espaço de Banach e $L \subset F$ um conjunto compacto, então todo homomorfismo contínuo $A: H_{b}(U) \rightarrow\left(\mathcal{H}(L), \tau_{w}\right)$ é um operador de composição. 
Demonstração. Sejam $\left(\mathcal{H}(L), \tau_{w}\right)=\lim _{\overrightarrow{m \in \mathbb{N}}} H_{b}\left(V_{m}\right)$, com $V_{m}=L+B_{\frac{1}{m}}(0)$ e a inclusão natural $\mathcal{I}_{m}^{L}: H_{b}\left(V_{m}\right) \rightarrow \mathcal{H}(L)$.

Do teorema 1.2.11 de Grothendieck, temos que existe um conjunto aberto $V_{m_{k}} \supset L$ e uma aplicação linear contínua $\varphi: H_{b}(U) \rightarrow H_{b}\left(V_{m_{k}}\right)$ tal que $\mathcal{I}_{m_{k}}^{L} \circ \varphi=A$.

Como cada $\mathcal{I}_{m}^{L}$ e $A$ são homomorfismos contínuos, temos que:

$$
[\varphi(f \cdot g)]=A(f \cdot g)=A(f) \cdot A(g)=[\varphi(f)][\varphi(g)]=[\varphi(f) \cdot \varphi(g)], \forall f, g \in H_{b}(U)
$$

então, $\varphi(f \cdot g) \sim \varphi(f) \cdot \varphi(g), \forall f, g \in H_{b}(U)$. Assim, existe $V_{m_{s}} \subset V_{m_{k}}$ tal que $\varphi(f \cdot g)_{\left.\right|_{m_{m_{s}}}}=(\varphi(f) \cdot \varphi(g))_{\left.\right|_{v_{m_{s}}}}$. Como $H_{b}\left(V_{m_{k}}\right) \stackrel{i_{m_{k}}^{L}}{\longrightarrow} H_{b}\left(V_{m_{s}}\right)$ temos que

$$
\left(i_{m_{k}}^{L} \circ \varphi\right)(f \cdot g)=\left(i_{m_{k}}^{L} \circ \varphi\right)(f) \cdot\left(i_{m_{k}}^{L} \circ \varphi\right)(g) .
$$

Assim podemos definir um homomorfismo contínuo $\psi: H_{b}(U) \rightarrow H_{b}\left(V_{m_{s}}\right)$ por $\psi=i_{m_{k}}^{L} \circ \varphi$ e como $\mathcal{I}_{m_{s}}^{L} \circ i_{m_{k}}^{L}=\mathcal{I}_{m_{k}}^{L}$ temos $A=\mathcal{I}_{m_{k}}^{L} \circ \varphi=\mathcal{I}_{m_{s}}^{L} \circ i_{m_{k}}^{L} \circ \varphi=\mathcal{I}_{m_{s}}^{L} \circ \psi$.

Do corolário 2.2.7, segue que $\psi: H_{b}(U) \rightarrow H_{b}\left(V_{m_{s}}\right)$ é um operador de composição, logo existe $\Phi \in H_{b}\left(V_{m_{s}}, E\right)$ tal que $\Phi\left(V_{m_{s}}\right) \subset U$ e $\psi(f)=f \circ \Phi, \forall f \in H_{b}(U)$.

Assim, existe $\Phi \in H_{b}\left(V_{m_{s}}, E\right)$ tal que $\Phi(L) \subset U$ e $A(f)=[f \circ \Phi], \forall f \in H_{b}(U)$. Isto é, $A=A_{\Phi}$.

Proposição 3.2.3 Sejam E um espaço de Tsirelson, $K \subset E$ um conjunto compacto convexo e equilibrado. Sejam $F$ um espaço de Banach e $V \subset F$ um conjunto aberto, então todo homomorfismo contínuo $A:\left(\mathcal{H}(K), \tau_{w}\right) \rightarrow H_{b}(V)$ é um operador de composição.

Demonstração. $\quad \operatorname{Sejam}\left(\mathcal{H}(K), \tau_{w}\right)=\lim _{n \in \mathbb{N}} H_{b}\left(U_{n}\right)$, com $U_{n}=K+B_{\frac{1}{n}}(0)$ e $\mathcal{I}_{n}^{K}$ : $H_{b}\left(U_{n}\right) \rightarrow \mathcal{H}(K)$ a inclusão natural. Como $K$ é convexo e equilibrado, temos que cada $U_{n}$ é um conjunto aberto convexo e equilibrado.

Como cada $\mathcal{I}_{n}^{K}$ e $A$ são homomorfismos contínuos, então $A \circ \mathcal{I}_{n}^{K}: H_{b}\left(U_{n}\right) \rightarrow H_{b}(V)$ é um homomorfismo contínuo. Do corolário 2.2.7, temos que $A \circ \mathcal{I}_{n}^{K}: H_{b}\left(U_{n}\right) \rightarrow H_{b}(V)$ é um operador de composição. Logo existe $\varphi_{n} \in H_{b}(V, E) \operatorname{com} \varphi_{n}(V) \subset U_{n}$ tal que $A \circ \mathcal{I}_{n}^{K}(f)=f \circ \varphi_{n}, \forall f \in H_{b}\left(U_{n}\right)$. 
Seja $\Phi=\varphi_{1}: V \rightarrow E \operatorname{com} \varphi_{1}(V) \subset U_{1}$. Então $\Phi \in H_{b}(V, E)$ e satisfaz $\Phi(V) \subset U_{1}$. Vamos agora mostrar que $\varphi_{n}=\varphi_{m}, \forall m, n \in \mathbb{N}$. Dado que $\left(E^{\prime},\|\|\right) \hookrightarrow H_{b}\left(U_{n}\right), \forall n \in \mathbb{N}$ temos que

$$
\left\{\begin{array}{l}
A\left(\left[x^{\prime}\right]\right)=x^{\prime} \circ \varphi_{n}, \forall x^{\prime} \in E^{\prime} \quad e \\
A\left(\left[x^{\prime}\right]\right)=x^{\prime} \circ \varphi_{m}, \forall x^{\prime} \in E^{\prime}, \forall m, n \in \mathbb{N}
\end{array}\right.
$$

$\operatorname{logo}, x^{\prime} \circ \varphi_{n}=x^{\prime} \circ \varphi_{m}, \forall x^{\prime} \in E^{\prime}$, pelo teorema de Hahn-Banach temos que

$$
\varphi_{n}=\varphi_{m}, \forall m, n \in \mathbb{N}
$$

Finalmente, mostraremos que $\Phi(V) \subset K$. Da equação (3.9) temos que $\Phi(V) \subset U_{n}, \forall n \in$ $\mathbb{N}$. Assim para cada $y \in V, \exists \xi_{n} \in K$ tal que $\left\|\Phi(y)-\xi_{n}\right\|<\frac{1}{n}$, para cada $n \in \mathbb{N}$, isto é, $d(\Phi(y), K)<\frac{1}{n}, \forall n \in \mathbb{N}, \forall y \in V$; fazendo $n \rightarrow \infty$, temos $d(\Phi(y), K)=0$, para cada $y \in V$. Desta forma, $\Phi(V) \subset K$.

Assim, existe $\Phi \in H_{b}(V, E)$ tal que $A([f])=f \circ \Phi, \forall f \in H_{b}\left(U_{n}\right)$. Isto é, $A=A_{\Phi}$.

\subsection{Homomorfismos compactos entre álgebras de ger- mes holomorfos}

Nesta seção estudaremos os homomorfismos compactos entre álgebras de germes de funções holomorfas.

No teorema 3.3.2 daremos uma condição necessária para que um homomorfismo contínuo entre álgebras de germes de funções holomorfas seja compacto. Na proposição 3.3.3 apresentaremos uma condição necessária e suficiente para que operadores de composição entre álgebras de germes de funções holomorfas sejam compactos.

No que segue vamos dar os conceitos de funções compactas e fracamente compactas. Observamos que esta definição 3.3.1 não é a definição usual de aplicação (fracamente) compacta. 
Definição 3.3.1 Sejam $E, F$ espaços localmente convexos, um operador $T: E \rightarrow F$ é (fracamente) compacto se leva conjuntos limitados de $E$ em conjuntos relativamente (fracamente) compactos de $F$.

O teorema que demonstramos a seguir nos fornece uma condição necessária para que homomorfismos contínuos entre álgebras de germes sejam compactos.

Teorema 3.3.2 Sejam E, $F$ espaços de Banach, $K \subset E$ e $L \subset F$ subconjuntos compactos. Seja $A:\left(\mathcal{H}(K), \tau_{w}\right) \rightarrow\left(\mathcal{H}(L), \tau_{w}\right)$ um homomorfismo contínuo compacto. Então para cada aberto $U_{n} \supset K$ existem um aberto $V_{m_{n}} \supset L$ e um homomorfismo contínuo compacto $\Psi_{n}: H_{b}\left(U_{n}\right) \rightarrow H_{b}\left(V_{m_{n}}\right)$ tal que $\mathcal{I}_{m_{n}}^{L} \circ \Psi_{n}=A \circ \mathcal{I}_{n}^{K}$.

Demonstração. Seja $U_{n} \subset E$ um conjunto aberto, pela proposição 3.1:1, existem um aberto $V_{m_{r_{n}}} \supset L$ e um homomorfismo contínuo $\widetilde{A}_{n}: H_{b}\left(U_{n}\right) \rightarrow H_{b}\left(V_{m_{r_{n}}}\right)$ tal que $\mathcal{I}_{m_{r_{n}}}^{L} \circ \widetilde{A}_{n}=A \circ \mathcal{I}_{n}^{K}$.

Agora, pela proposição 1.3 .5 , temos que existe $m_{n}>m_{r_{n}}$ tal que

$$
\left(\mathcal{H}(L), \tau_{w}\right)_{\left.\right|_{B}}=H_{b}\left(V_{m_{n}}\right)_{\left.\right|_{B}} \text {, para cada conjunto limitado } B \text { de } H_{b}\left(V_{m_{r_{n}}}\right)
$$

Como $H_{b}\left(V_{m_{r_{n}}}\right) \stackrel{i_{m_{r_{n}}}^{L}}{\leftrightarrow} H_{b}\left(V_{m_{n}}\right)$ e $\mathcal{I}_{m_{n}}^{L} \circ i_{m_{r_{n}}}^{L}=\mathcal{I}_{m_{r_{n}}}^{L}$, podemos definir um homomorfismo contínuo $\Psi_{n}: H_{b}\left(U_{n}\right) \rightarrow H_{b}\left(V_{m_{n}}\right)$ por $\Psi_{n}=i_{m_{r_{n}}}^{L} \circ \widetilde{A}_{n}$ e $\operatorname{assim} A \circ \mathcal{I}_{n}^{K}=\mathcal{I}_{m_{n}}^{L} \circ \Psi_{n}$. Como mostra o seguinte diagrama comutativo.

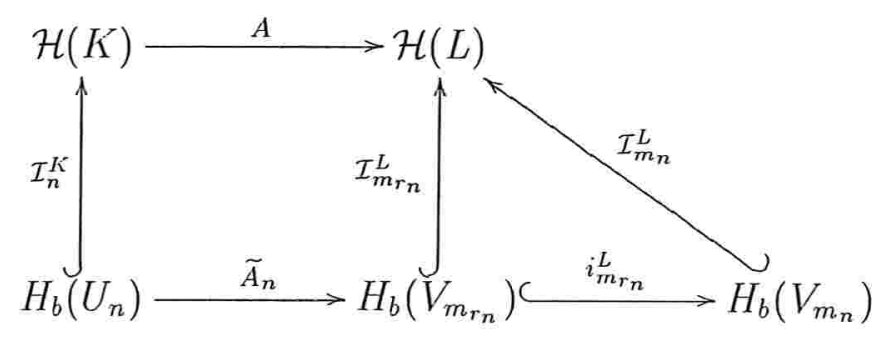

Afirmamos que o homomofismo $\Psi_{n}$ é compacto. De fato, seja $\mathcal{X} \subset H_{b}\left(U_{n}\right)$ limitado, $\operatorname{logo} \widetilde{A}_{n}(\mathcal{X}) \subset H_{b}\left(V_{m_{r_{n}}}\right)$ é limitado. Como $A$ é compacto, segue que $\mathcal{I}_{m_{r_{n}}}^{L}\left(\widetilde{A}_{n}(\mathcal{X})\right) \subset \mathcal{H}(L)$ é relativamente compacto e como $\Psi_{n}(\mathcal{X})=i_{m_{r_{n}}}^{L}\left(\widetilde{A}_{n}(\mathcal{X})\right) \subset H_{b}\left(V_{m_{n}}\right)$ segue de $(3.10)$ que é relativamente compacto. Logo $\Psi_{n}$ é compacto. 
Assim, para cada aberto $U_{n} \supset K$ existem um aberto $V_{m_{n}} \supset L$ e um homomorfismo compacto $\Psi_{n}: H_{b}\left(U_{n}\right) \rightarrow H_{b}\left(V_{m_{n}}\right)$ tal que $\mathcal{I}_{m_{n}}^{L} \circ \Psi_{n}=A \circ \mathcal{I}_{n}^{K}$.

A seguir demonstraremos uma proposição que nos fornece uma condição necessária e suficiente para que operadores de composição sejam compactos.

Proposição 3.3.3 Sejam E, $F$ espaços de Banach, $K \subset E$ e $L \subset F$ subconjuntos compactos. Seja $A:\left(\mathcal{H}(K), \tau_{w}\right) \rightarrow\left(\mathcal{H}(L), \tau_{w}\right)$ um operador de composição. Então $A$ é compacto se, e somente se, para cada aberto $U_{n} \supset K$ existem um aberto $V_{m_{n}} \supset L$ e um operador de composição compacto $\Psi_{n}: H_{b}\left(U_{n}\right) \rightarrow H_{b}\left(V_{m_{n}}\right)$ tal que $\mathcal{I}_{m_{n}}^{L} \circ \Psi_{n}=A \circ \mathcal{I}_{n}^{K}$.

Demonstração. Vamos primeiramente supor que $A$ é compacto. Vamos mostrar que para cada aberto $U_{n} \supset K$ existem um aberto $V_{m_{n}} \supset L$ e um operador de composição compacto $\Psi_{n}: H_{b}\left(U_{n}\right) \rightarrow H_{b}\left(V_{m_{n}}\right)$ tal que $\mathcal{I}_{m_{n}}^{L} \circ \Psi_{n}=A \circ \mathcal{I}_{n}^{K}$.

De fato, segue do teorem 3.1.3 que para cada aberto $U_{n} \supset K$ existem um aberto $V_{m_{r_{n}}} \supset L$ e um operador de composição $\widetilde{A}_{n}: H_{b}\left(U_{n}\right) \rightarrow H_{b}\left(V_{m_{r_{n}}}\right)$ tal que $\mathcal{I}_{m_{r_{n}}}^{L} \circ \widetilde{A}_{n}=$ $A \circ \mathcal{I}_{n}^{K}$. Agora, pela construção do teorema 3.3.2 temos existem um conjunto aberto $V_{m_{n}} \supset L \operatorname{com} H_{b}\left(V_{m_{r_{n}}}\right) \stackrel{i_{m_{r_{n}}}^{L}}{\longrightarrow} H_{b}\left(V_{m_{n}}\right)$ e um operador de composição compacto $\Psi_{n}=$ $i_{m_{r_{n}}}^{L} \circ \widetilde{A}_{n}: H_{b}\left(U_{n}\right) \rightarrow H_{b}\left(V_{m_{n}}\right)$ tal que $\mathcal{I}_{m_{n}}^{L} \circ \Psi_{n}=A \circ \mathcal{I}_{n}^{K}$.

Reciprocamente, suponhamos que para cada aberto $U_{n} \supset K$ existem um aberto $V_{m_{n}} \supset L$ e um operador de composição compacto $\Psi_{n}: H_{b}\left(U_{n}\right) \rightarrow H_{b}\left(V_{m_{n}}\right)$ tal que $\mathcal{I}_{m_{n}}^{L} \circ \Psi_{n}=A \circ \mathcal{I}_{n}^{K}$. Vamos provar que $A$ é compacto.

De fato, seja $\mathcal{X} \subset \mathcal{H}(K)$ um conjunto limitado. Vamos mostrar que $A(\mathcal{X}) \subset \mathcal{H}(L)$ é relativamente compacto. Da proposição 1.3 .5 sabemos que existe $r \in \mathbb{N}$ tal que $\mathcal{X} \subset$ $H_{b}\left(U_{r}\right)$ é limitado.

Por hipótese, existem um conjunto aberto $V_{m_{r}} \supset L$ e um operador de composição compacto $\Psi_{r}: H_{b}\left(U_{r}\right) \rightarrow H_{b}\left(V_{m_{r}}\right)$ tal que $\mathcal{I}_{m_{r}}^{L} \circ \Psi_{r}=A \circ \mathcal{I}_{r}^{K} . \operatorname{Assim}, \Psi_{r}(\mathcal{X}) \subset H_{b}\left(V_{m_{r}}\right)$ é relativamente compacto, da continuidade de $\mathcal{I}_{m_{r}}^{L}$ concluimos que $A(\mathcal{X})=\mathcal{I}_{m_{r}}^{L}\left(\Psi_{r}(\mathcal{X})\right) \subset$ $\mathcal{H}(L)$ é relativamente compacto. Assim, $A$ é compacto. 
Exemplo 3.3.4 Sejam $F$ um espaço de Banach reflexivo, $\Phi: F \rightarrow \ell_{1}$ uma aplicação linear contínua. Se $L \subset F$ é um subconjunto compacto e $K=\Phi(L)$, então o operador de composição $A_{\Phi}:\left(\mathcal{H}(K), \tau_{w}\right) \rightarrow\left(\mathcal{H}(L), \tau_{w}\right)$ é compacto.

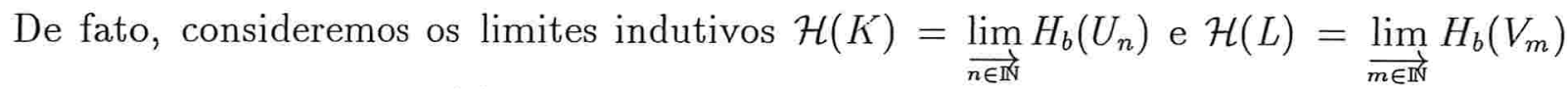
onde cada $U_{n}=K+B_{\frac{1}{n}}(0)$ e $V_{m}=L+B_{\frac{1}{m}}(0)$.

Dado que $A_{\Phi}$ é um operador de composição, para cada aberto $U_{n} \supset L$ existem, pelo teorema 3.1.3, um aberto $V_{m_{n}} \supset L$ e um operador de composição $\widetilde{A}_{n_{\Phi_{n}}}: H_{b}\left(U_{n}\right) \rightarrow$ $H_{b}\left(V_{m_{n}}\right)$ tal que $\mathcal{I}_{m_{n}}^{L} \circ \widetilde{A}_{n_{\Phi_{n}}}=A_{\Phi} \circ \mathcal{I}_{n}^{L}$ e $\Phi_{n}=\Phi_{l_{v_{m_{n}}}}$. Como, $\Phi$ é uma aplicação compacta segue que $\Phi_{n}$ é uma aplicação compacta. Pela proposição 13 de [13], segue que $\widetilde{A}_{n_{\phi_{n}}}$ é compacto. Assim, pela proposição 3.3 .3 temos que $A_{\Phi}$ é compacto.

\subsection{Operadores de composição fracamente compac- tos entre espaços de germes}

No teorema 3.4.1 damos uma condição necessária para que um homomorfismo contínuo entre álgebras de germes holomorfos seja fracamente compacto. Na proposição 3.4 .2 apresentamos uma condição necessária e suficiente para operadores de composição entre álgebras de germes holomorfos sejam fracamente compactos.

Teorema 3.4.1 Sejam $E, F$ espaços de Banach, $K \subset E$ e $L \subset F$ subconjuntos compactos. Seja $A:\left(\mathcal{H}(K), \tau_{w}\right) \rightarrow\left(\mathcal{H}(L), \tau_{w}\right)$ um homomorfismo contínuo fracamente compacto. Então para cada aberto $U_{n} \supset K$ existem um aberto $V_{m_{n}} \supset L$ e um homomorfismo contínuo fracamente compacto $\Psi_{n}: H_{b}\left(U_{n}\right) \rightarrow H_{b}\left(V_{m_{n}}\right)$ tal que $\mathcal{I}_{m_{n}}^{L} \circ \Psi_{n}=A \circ \mathcal{I}_{n}^{K}$.

Demonstração. Seja $U_{n} \subset E$ um conjunto aberto, pela proposição 3.1.1, existem um aberto $V_{m_{r_{n}}} \supset L$ e um homomorfismo contínuo $\widetilde{A}_{n}: H_{b}\left(U_{n}\right) \rightarrow H_{b}\left(V_{m_{r_{n}}}\right)$ tal que $\mathcal{I}_{m_{r_{n}}}^{L} \circ \tilde{A}_{n}=A \circ \mathcal{I}_{n}^{K}$. 
Agora, pelo corolário 1.3.6, existe $m_{n}>m_{r_{n}}$ tal que

$$
\sigma\left(\mathcal{H}(L), \mathcal{H}(L)^{\prime}\right)_{\left.\right|_{B}}=\sigma\left(H_{b}\left(V_{m_{n}}\right), H_{b}\left(V_{m_{n}}\right)^{\prime}\right)_{\left.\right|_{B}}
$$

para cada conjunto limitado $B$ de $H_{b}\left(V_{m_{r_{n}}}\right)$. Como $H_{b}\left(V_{m_{r_{n}}}\right) \stackrel{i_{m_{r_{n}}}^{L}}{\hookrightarrow} H_{b}\left(V_{m_{n}}\right)$ e $\mathcal{I}_{m_{n}}^{L} \circ i_{m_{r_{n}}}^{L}=$ $\mathcal{I}_{m_{r_{n}}}^{L}$, podemos definir um homomorfismo contínuo $\Psi_{n}: H_{b}\left(U_{n}\right) \rightarrow H_{b}\left(V_{m_{n}}\right)$ por $\Psi_{n}=$ $i_{m_{r_{n}}}^{L} \circ \widetilde{A}_{n}$ e $\operatorname{assim} A \circ \mathcal{I}_{n}^{K}=\mathcal{I}_{m_{n}}^{L} \circ \Psi_{n}$. Como mostra o seguinte diagrama comutativo.

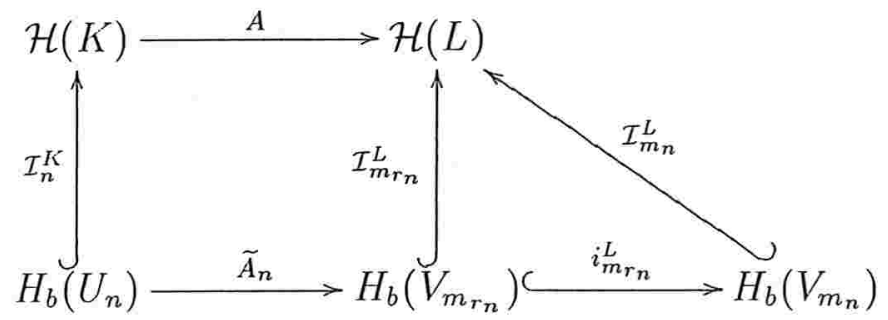

Afirmamos que o homomorfismo $\Psi_{n}$ é fracamente compacto. De fato, seja $\mathcal{X} \subset$ $H_{b}\left(U_{n}\right)$ limitado, logo $\widetilde{A}_{n}(\mathcal{X}) \subset H_{b}\left(V_{m_{r_{n}}}\right)$ é limitado. Como $A$ é fracamente compacto, segue que $\mathcal{I}_{m_{r_{n}}}^{L}\left(\widetilde{A}_{n}(\mathcal{X})\right) \subset \mathcal{H}(L)$ é relativamente fracamente compacto e como $\Psi_{n}(\mathcal{X})=$ $i_{m_{r_{n}}}^{L}\left(\widetilde{A}_{n}(\mathcal{X})\right) \subset H_{b}\left(V_{m_{n}}\right)$ segue por (3.11) que é relativamente fracamente compacto. Logo $\Psi_{n}$ é fracamente compacto.

Assim, para cada aberto $U_{n} \supset K$ existem um aberto $V_{m_{n}} \supset L$ e um homomorfismo fracamente compacto $\Psi_{n}: H_{b}\left(U_{n}\right) \rightarrow H_{b}\left(V_{m_{n}}\right)$ tal que $\mathcal{I}_{m_{n}}^{L} \circ \Psi_{n}=A \circ \mathcal{I}_{n}^{K}$.

A seguir demonstraremos a proposição que nos fornece uma condição necessária e suficiente para que operadores de composição entre espaços de germes holomorfos sejam fracamente compactos.

Proposição 3.4.2 Sejam E, $F$ espaços de Banach, $K \subset E$ e $L \subset F$ subconjuntos compactos. Seja $A:\left(\mathcal{H}(K), \tau_{w}\right) \rightarrow\left(\mathcal{H}(L), \tau_{w}\right)$ um operador de composição. Então $A$ é fracamente compacto se, e somente se, para cada aberto $U_{n} \supset K$ existem um aberto $V_{m_{n}} \supset L$ e um operador de composição fracamente compacto $\Psi_{n}: H_{b}\left(U_{n}\right) \rightarrow H_{b}\left(V_{m_{n}}\right)$ tal que $\mathcal{I}_{m_{n}}^{L} \circ \Psi_{n}=A \circ \mathcal{I}_{n}^{K}$.

Demonstração. Vamos primeiramente supor que $A$ é fracamente compacto. Vamos mostrar que para cada aberto $U_{n} \supset K$ existem um aberto $V_{m_{n}} \supset L$ e um operador de 
composição fracamente compacto $\Psi_{n}: H_{b}\left(U_{n}\right) \rightarrow H_{b}\left(V_{m_{n}}\right)$ tal que $\mathcal{I}_{m_{n}}^{L} \circ \Psi_{n}=A \circ \mathcal{I}_{n}^{K}$.

De fato, segue do teorema 3.1.3 que para cada aberto $U_{n} \supset K$ existem um aberto $V_{m_{r_{n}}} \supset L$ e um operador de composição $\widetilde{A}_{n}: H_{b}\left(U_{n}\right) \rightarrow H_{b}\left(V_{m_{r_{n}}}\right)$ tal que $\mathcal{I}_{m_{r_{n}}}^{L} \circ \widetilde{A}_{n}=$ $A \circ \mathcal{I}_{n}^{K}$. Agora, pela mesma construção do teorema 3.4.1 temos que existem um conjunto aberto $V_{m_{n}} \supset L \operatorname{com} H_{b}\left(V_{m_{r_{n}}}\right) \stackrel{i_{m_{r_{n}}}^{L}}{\longrightarrow} H_{b}\left(V_{m_{n}}\right)$ e um operador de composição fracamente compacto $\Psi_{n}=i_{m_{r_{n}}}^{L} \circ \widetilde{A}_{n}: H_{b}\left(U_{n}\right) \rightarrow H_{b}\left(V_{m_{n}}\right)$ tal que $\mathcal{I}_{m_{n}}^{L} \circ \Psi_{n}=A \circ \mathcal{I}_{n}^{K}$.

Reciprocamente, suponhamos que para cada aberto $U_{n} \supset K$ existem um aberto $V_{m_{n}} \supset L$ e um operador de composição fracamente compacto $\Psi_{n}: H_{b}\left(U_{n}\right) \rightarrow H_{b}\left(V_{m_{n}}\right)$ tal que $\mathcal{I}_{m_{n}}^{L} \circ \Psi_{n}=A \circ \mathcal{I}_{n}^{K}$. Vamos provar que $A$ é fracamente compacto.

De fato, seja $\mathcal{X} \subset \mathcal{H}(K)$ um conjunto limitado. Vamos mostrar que $A(\mathcal{X}) \subset \mathcal{H}(L)$ é relativamente fracamente compacto. Da proposição 1.3 .5 sabemos que existe $r \in \mathbb{N}$ tal que $\mathcal{X} \subset H_{b}\left(U_{r}\right)$ é limitado.

Pela hipótese, existem um conjunto aberto $V_{m_{r}} \supset L$ e um operador de composição fracamente compacto $\Psi_{r}: H_{b}\left(U_{r}\right) \rightarrow H_{b}\left(V_{m_{r}}\right)$ tal que $\mathcal{I}_{m_{r}}^{L} \circ \Psi_{r}=A \circ \mathcal{I}_{r}^{K}$. Assim $\Psi_{r}(\mathcal{X}) \subset$ $H_{b}\left(V_{m_{r}}\right)$ é relativamente fracamente compacto, da continuidade de $\mathcal{I}_{m_{r}}^{L}$ concluimos que $A(\mathcal{X})=\mathcal{I}_{m_{r}}^{L}\left(\Psi_{r}(\mathcal{X})\right) \subset \mathcal{H}(L)$ é relativamente fracamente compacto.

Portanto, $A$ é fracamente compacto.

Exemplo 3.4.3 Sejam $E$ um espaço de Tsirelson e $\Phi: E \rightarrow E$ dada por $\Phi(z)=\frac{z}{2}, \forall z \in$ $E$ uma aplicação holomorfa. Se $K \subset E$ é um subconjunto compacto, equilibrado, então o operador de composição $A_{\Phi}:\left(\mathcal{H}(K), \tau_{w}\right) \rightarrow\left(\mathcal{H}(K), \tau_{w}\right)$ é fracamente compacto, mas não é compacto.

De fato, consideremos o limite indutivo $\mathcal{H}(K)=\underset{n \in \mathbb{N}}{\lim } H_{b}\left(U_{n}\right)$ onde cada $U_{n}=K+B_{\frac{1}{n}}(0)$.

Observamos que, se $m_{n} \geq n$ então $\Phi\left(U_{m_{n}}\right)$ é $U_{n}$-limitado. Basta considerar $\Phi\left(U_{m_{n}}\right)+\epsilon B_{\epsilon}(0) \subset U_{n} \operatorname{com} \epsilon<\frac{1}{2 m_{n}}$.

Vamos primeiramente mostrar que $A_{\Phi}$ é um operador de composição fracamente compacto. 
Dado que $A_{\Phi}$ é um operador de composição, para cada aberto $U_{n} \supset K$ existem, pelo teorema 3.1.3, um aberto $U_{m_{n}} \supset K$ (podemos escolher $m_{n} \geq n$ ) e um operador de composição $\widetilde{A}_{n_{\phi_{n}}}: H_{b}\left(U_{n}\right) \rightarrow H_{b}\left(U_{m_{n}}\right)$ tal que $\mathcal{I}_{m_{n}}^{K} \circ \widetilde{A}_{n_{\Phi_{n}}}=A_{\Phi} \circ \mathcal{I}_{n}^{K}$. Além disso, pela observação anterior $\Phi_{n}\left(U_{m_{n}}\right)$ é $U_{n}$-limitado.

Como $E$ é um espaço de Tsirelson, $H_{b}\left(U_{n}\right)$ é reflexivo e consequentemente $\widetilde{A}_{n_{\phi_{n}}}$ é fracamente compacto, segue da proposição 3.4 .2 , que $A_{\Phi}$ é fracamente compacto.

Agora, vamos mostrar que $A_{\Phi}$ não é compacto. Suponha por absurdo que $A_{\Phi}$ é compacto, segue da proposição 3.3 .3 que $\widetilde{A}_{n_{\phi_{n}}}$ é compacto e, pela proposição 13 de [13], temos que $\Phi_{n}$ é compacto. Como $\Phi\left(U_{m_{n}}\right)$ é $U_{n}$-limitado, temos que $\frac{1}{2} B_{E} \subset \overline{\Phi_{n}\left(U_{m_{n}}\right)} \subset U_{n}$ é compacto, assim $\operatorname{dim}(E)<\infty$ ( absurdo! ). Portanto, $A_{\Phi}$ não é compacto.

Observamos que, se consideramos $L \subset E$ um subconjunto compacto e $K=\Phi(L)$, no exemplo 3.4.3, temos que $A_{\Phi}:\left(\mathcal{H}(K), \tau_{w}\right) \rightarrow\left(\mathcal{H}(L), \tau_{w}\right)$ é fracamente compacto.

Estamos interessados em determinar condições para que operadores de composição fracamente compactos entre álgebras de germes holomorfos sejam compactos. Neste sentido, vamos apresentar a seguinte definição.

Denotamos por $(E, \sigma(E, P(E)))$ o conjunto $E$ munido da topologia menos fina que torna todos os polinômios $P \in \mathcal{P}(E)$ contínuos. A topologia $\sigma(E, P(E))$ é regular e Hausdorff tal que $(E,\|\|) \geq(E, \sigma(E, P(E))) \geq\left(E, \sigma\left(E, E^{\prime}\right)\right)$.

Definição 3.4.4 Um espaço de Banach E é $\Lambda$-espaço, se todas as seqüências convergentes em $(E, \sigma(E, P(E)))$ são convergentes em norma.

A seguir demonstramos a proposição 3.4 .5 que nos fornece uma condição necessária para que operadores de composição entre espaços de germes holomorfos fracamente compactos sejam compactos.

Proposição 3.4.5 Sejam E um $\Lambda$-espaço com a propriedade de aproximação, $F$ um espaço de Banach, $K \subset E$ um subconjunto compacto, equilibrado e absolutamente convexo 
e $L \subset F$ subconjunto compacto. Seja $A:\left(\mathcal{H}(K), \tau_{w}\right) \rightarrow\left(\mathcal{H}(L), \tau_{w}\right)$ um operador de composição. Se A é fracamente compacto, então A é compacto.

Demonstração. Seja $A=A_{\Phi}$ um operador de composição fracamente compacto, segue da proposição 3.4 .2 que para cada aberto $U_{n} \supset K$ existem $V_{m_{n}} \supset L$ aberto e $\widetilde{A}_{n_{\Phi_{n}}}: H_{b}\left(U_{n}\right) \rightarrow H_{b}\left(V_{m_{n}}\right)$ um operador de composição fracamente compacto tal que $\mathcal{I}_{m_{n}}^{L} \circ \widetilde{A}_{n_{\Phi_{n}}}=A_{\Phi} \circ \mathcal{I}_{n}^{K}$.

Como cada $U_{n}=K+B_{\frac{1}{n}}(0)$ é equilibrado e absolutamente convexo, pela proposição 9 de [13], temos que $\Phi_{n}$ aplica conjuntos $V_{m_{n}}$-limitados em conjuntos $\sigma(E, P(E)$ )relativamente compactos de $U_{n} \subset E$. Como a topologia $\sigma(E, \mathcal{P}(E)$ ) é angélica, (ver 3.11 corolário 1 de [9]), segue que $\Phi_{n}$ aplica conjuntos $V_{m_{n}}$-limitados em sequencialmente $\sigma(E, \mathcal{P}(E))$-compactos. Usando a hipótese que $E$ é $\Lambda$-espaço, temos que $\Phi_{n}$ aplica conjuntos $V_{m_{n}}$-limitados em relativamente compactos. Consequentemente $\Phi_{n}$ é compacto e pela proposição 13 de [13] temos que $\widetilde{A}_{n_{\Phi_{n}}}: H_{b}\left(U_{n}\right) \rightarrow H_{b}\left(V_{m_{n}}\right)$ é compacto.

Agora, segue da proposição 3.3 .3 que $A_{\Phi}$ é compacto. 


\section{Capítulo 4}

\section{Homomorfismos contínuos entre álgebras de germes holomorfos a valores vetoriais}

Neste capítulo estudamos os homomorfismos contínuos entre álgebras de germes de funções holomorfas a valores em uma álgebra de Banach. Apresentamos uma caracterização para que tais homomorfismos sejam operadores de composição. A partir daí, passamos a estudar a compacidade e compacidade fraca de operadores de composição entre álgebras de germes de funções holomorfas a valores em uma álgebra de Banach.

Estamos usando os conceitos de funções compactas e fracamente compactas definidos em 3.3.1.

\subsection{Operadores de composição entre álgebras de funções holomorfas a valores vetoriais}

Nesta seção estudamos inicialmente os homomorfismos compactos e fracamente compactos entre álgebras de funções holomorfas a valores em uma álgebra de Banach. Em seguida 
passamos a estudar a compacidade e compacidade fraca de operadores de composição entre álgebras de funções holomorfas a valores em uma álgebra de Banach. Estes resultados serão usados no estudo de operadores de composição compactos e fracamente compactos entre espaços de germes de funções holomorfas. Para tal, usamos algumas aplicações do tipo avaliação que definimos na proposição 4.1.2 e apresentamos algumas propriedades.

Sejam $E$ um espaço de Banach, $G$ uma álgebra de Banach e $U \subset E$ um conjunto aberto. Denotamos por $H_{b}(U, G)$ a álgebra de Fréchet de todas as aplicações holomorfas de $U$ em $G$ que são limitadas sobre conjuntos $U$-limitados munida da topologia $\tau_{b}$ da convergência uniforme sobre os $U$-limitados.

Usamos as seguintes notações para funções holomorfas.

Notações 4.1.1 Sejam E, $F$ espaços de Banach, $U \subset E$ um conjunto aberto, $f \in$ $H(U, F)$ e $a \in U$. Sabemos que existe $B_{r}(a) \subset U$ e uma seqüência de polinômios $P_{m} \in \mathcal{P}\left({ }^{m} E, F\right)$ tal que $f(x)=\sum_{m=0}^{\infty} P_{m}(x-a)$ uniformemente para cada $x \in B_{r}(a)$.

A seqüência $\left(P_{m}\right)_{m}$ é unicamente determinada for $f$ e assim podemos escrever $P_{m}=$ $P^{m} f(a)$ para cada $m \in \mathbb{N}$.

A série $\sum_{m=0}^{\infty} P^{m} f(a)(x-a)$ é denominada a série de Taylor de $f$ em a. Denotamos por $A^{m} f(a)$ ao único elemento de $\mathcal{L}_{s}\left({ }^{m} E, F\right)$ tal que

$$
P^{m} f(a)(x)=A^{m} f(a)\left(x^{m}\right)=\widehat{A}^{m} f(a)(x)
$$

Outra notação que usamos é:

(a) $d^{m} f(a)=m ! A^{m} f(a) \in \mathcal{L}_{s}\left({ }^{m} E, F\right)$,

(b) $\widehat{d}^{m} f(a)=m ! \widehat{A}^{m} f(a)=m ! P^{m} f(a) \in \mathcal{P}\left({ }^{m} E, F\right)$

Proposição 4.1.2 Sejam E, $G$ espaços de Banach, $V \subset E$ um conjunto aberto, $a \in G e$ $a^{\prime} \in G^{\prime} \operatorname{com}\|a\|=1,\left\|a^{\prime}\right\|=1$ e $a^{\prime}(a)=1$. Então: 
(i) $J_{a}:\left(E^{\prime},\|\|\right) \rightarrow H_{b}(E, G)$ dada por $J_{a}\left(x^{\prime}\right)(x)=x^{\prime}(x) a, \forall x^{\prime} \in E^{\prime}, \forall x \in E$ é uma aplicação linear e contínua. Além disso, a aplicação $J_{a}^{\prime}:\left(H_{b}(E, G)^{\prime}, \tau_{\beta}\right) \rightarrow\left(E^{\prime \prime},\|\|\right)$ é contínua (onde $\tau_{\beta}$ é a topologia forte).

(ii) $\delta_{a^{\prime}}: V \rightarrow\left(H_{b}(V, G)^{\prime}, w^{*}\right)$ dada por $\delta_{a^{\prime}}(y)(g)=a^{\prime}(g(y)), \forall y \in V, \forall g \in H_{b}(V, G)$ é contínua e leva conjuntos $V$-limitados de $V$ em conjuntos fortemente limitados de $H_{b}(V, G)^{\prime}$.

\section{Demonstração.}

(i) $J_{a}$ assim definida é linear. Vamos provar que é contínua na origem. Seja $V_{0, B, \epsilon} \in$ $\mathcal{B}_{H_{b}(E, G)}(0)$, onde $B \subset E$ é limitado. Assim $\exists \lambda_{B}>0$ tal que $\|x\| \leq \lambda_{B}, \forall x \in B$. Seja $x^{\prime} \in$ $E^{\prime}$ com $\left\|x^{\prime}\right\|<\frac{\epsilon}{\lambda_{B}}$, como $\left\|J_{a}\left(x^{\prime}\right)(x)\right\|=\left\|x^{\prime}(x) a\right\|$ temos que $\left\|J_{a}\left(x^{\prime}\right)\right\|_{B} \leq\left\|x^{\prime}\right\| \sup _{x \in B}\|x\|<\epsilon$. Assim $J_{a}\left(x^{\prime}\right) \in V_{0, B, \epsilon}$. Portanto, $J_{a}:\left(E^{\prime},\|\|\right) \rightarrow H_{b}(E, G)$ é contínua.

Agora, $J_{a}^{\prime}:\left(H_{b}(E, G)^{\prime}, \tau_{\beta}\right) \rightarrow\left(E^{\prime \prime},\|\|\right)$ é contínua, pois se considero $B_{\epsilon}^{\prime \prime}(0) \in \mathcal{B}_{E^{\prime \prime}}(0)$,

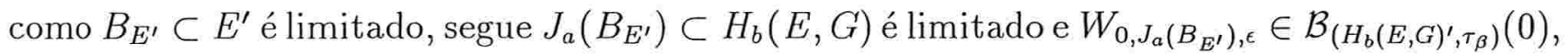
assim se $f^{\prime} \in W_{0, J_{a}\left(B_{E^{\prime}}\right), \epsilon}$ temos $\left|f^{\prime}\left(J_{a}\left(x^{\prime}\right)\right)\right|<\epsilon, \forall x^{\prime} \in B_{E^{\prime}} \operatorname{logo} J_{a}^{\prime}\left(f^{\prime}\right) \in B_{\epsilon}^{\prime \prime}(0)$. Portanto, $J_{a}^{\prime}$ é contínua.

(ii) $\delta_{a^{\prime}}$ é contínua. Seja $y_{0} \in V$ e $V_{\delta_{a^{\prime}}\left(y_{0}\right), g_{1}, \cdots, g_{r}, \epsilon} \in \mathcal{B}_{\left(H_{b}(V, G)^{\prime}, w^{*}\right)}\left(\delta_{a^{\prime}}\left(y_{0}\right)\right) \operatorname{com} g_{i} \in$ $H_{b}(V, G)$. Como cada $g_{i}$ é contínua em $y_{0} \in V$, existe $B_{\lambda_{i}}\left(y_{0}\right) \subset V$ tal que para cada $y \in B_{\lambda_{i}}\left(y_{0}\right)$ temos $\left\|g_{i}(y)-g_{i}\left(y_{0}\right)\right\| \leq \epsilon$.

Sejam $\lambda=\min _{1 \leq i \leq r}\left\{\lambda_{i}\right\}$ e $y \in B_{\lambda}\left(y_{0}\right)$. Logo $y \in B_{\lambda_{i}}\left(y_{0}\right)$ para cada $i=1, \cdots, r$. Então $\left\|\delta_{a^{\prime}}(y)\left(g_{i}\right)-\delta_{a^{\prime}}\left(y_{0}\right)\left(g_{i}\right)\right\|=\left\|g_{i}(y)-g_{i}\left(y_{0}\right)\right\|<\epsilon$ para cada $i=1, \cdots, r$, assim $\delta_{a^{\prime}}(y) \in V_{\delta_{a^{\prime}}\left(y_{0}\right), g_{1}, \cdots, g_{r}, \epsilon}$. Portanto, $\delta_{a^{\prime}}$ é contínua em $y_{0}$ arbitrário e consequentemente contínua.

Vamos agora mostrar que $\delta_{a^{\prime}}$ aplica conjuntos $V$-limitados de $V$ em conjuntos fortemente limitados de $H_{b}(V, G)^{\prime}$.

De fato, sejam $B \subset V$ um conjunto $V$-limitado e $V_{0, g_{1}, \cdots, g_{r}, \epsilon} \in \mathcal{B}_{\left(H_{b}(V, G)^{\prime}, w^{*}\right)}(0)$ com $g_{i} \in H_{b}(V, G)$. Assim $g_{i}(B) \subset G$ é limitado e portanto $\exists \lambda_{i}>0$ tal que $\sup _{y \in B}\left\|g_{i}(y)\right\|<\lambda_{i}$.

Sejam $\lambda=\max _{1 \leq i \leq r}\left\{\lambda_{i}\right\}$ e $g^{\prime} \in \frac{\epsilon}{2 \lambda} \delta_{a^{\prime}}(B)$ então $\left|g^{\prime}\left(g_{i}\right)\right|<\epsilon, i=1, \cdots, r$ e $g^{\prime} \in V_{0, g_{1}, \cdots, g_{r}, \epsilon}$. 
Consequentemente $\frac{\epsilon}{2 \lambda} \delta_{a^{\prime}}(B) \subset V_{0, g_{1}, \cdots, g_{r}, \epsilon}$ e é $w^{*}$-limitado. Como $H_{b}(V, G)$ é tonelado, segue que $\delta_{a^{\prime}}(B)$ é fortemente limitado para cada conjunto $B V$-limitado.

A seguir vamos apresentar um resultado para operadores de composição compactos entre certas álgebras de funções holomorfas.

Definição 4.1.3 Sejam E, $F$ espaços de Banach e $U \subset E$ um conjunto aberto. Uma aplicação $g: U \rightarrow F$ é (fracamente) compacto se leva conjuntos U-limitados em conjuntos relativamente (fracamente) compactos.

Teorema 4.1.4 Sejam E, F espaços de Banach e $G$ uma álgebra de Banach. Sejam $V \subset F$ um conjunto aberto, $\Phi \in H_{b}(V, E)$ e $T_{\Phi}: H_{b}(E, G) \rightarrow H_{b}(V, G)$ um operador de composição. Considere as seguintes afirmações:

(a) $T_{\Phi}$ é compacto;

(b) o adjunto $T_{\Phi}^{\prime}:\left(H_{b}(V, G)^{\prime}, \tau_{\beta}\right) \rightarrow\left(H_{b}(E, G)^{\prime}, \tau_{\beta}\right)$ é compacto, onde $\tau_{\beta}$ a topologia forte;

(c) $\Phi$ é uma aplicação compacta.

Então $(a) \Rightarrow(b) \Rightarrow(c)$.

Demonstração. $\quad(a) \Rightarrow(b)$ Vamos primeiro mostrar que $T_{\Phi}^{\prime}:\left(H_{b}(V, G)^{\prime}, \tau_{c}\right) \rightarrow$ $\left(H_{b}(E, G)^{\prime}, \tau_{\beta}\right)$ é contínua, onde $\tau_{c}$ é a topologia da convergência uniforme sobre os conjuntos compactos de $H_{b}(V, G)$ e $\tau_{\beta}$ a topologia forte.

De fato, seja $V_{0, \mathcal{X}, \epsilon}=\left\{f^{\prime} \in H_{b}(E, G)^{\prime} /\left\|f^{\prime}\right\|_{\mathcal{X}}<\epsilon\right\} \in \mathcal{B}_{\left(H_{b}(E, G)^{\prime}, \tau_{\beta}\right)}(0), \operatorname{com} \mathcal{X} \subset$ $H_{b}(E, G)$ limitado. Como $T_{\Phi}: H_{b}(E, G) \rightarrow H_{b}(V, G)$ é compacto temos que $T_{\Phi}(\mathcal{X}) \subset$ $H_{b}(V, G)$ é relativamente compacto. Seja $\Gamma\left(\overline{T_{\Phi}(\mathcal{X})}\right)$ a envoltoria absolutamente convexa e fechada, como $H_{b}(V, G)$ é Fréchet, temos que $\Gamma\left(\overline{T_{\Phi}(\mathcal{X})}\right)$ é compacto.

Considere $W_{0, \Gamma\left(\overline{T_{\Phi}(\mathcal{X})}\right), \epsilon}=\left\{h^{\prime} \in H_{b}(V, G)^{\prime} /\left\|h^{\prime}\right\|_{\Gamma\left(\overline{T_{\Phi}(\mathcal{X})}\right)}<\epsilon\right\} \in \mathcal{B}_{\left(H_{b}(V, G)^{\prime}, T_{c}\right)}(0)$. 
Vamos mostrar que $T_{\Phi}^{\prime}\left(W_{0, \Gamma\left(\overline{T_{\Phi}(\mathcal{X})}\right), \epsilon}\right) \subset V_{0, \mathcal{X}, \epsilon}$. Seja $h^{\prime} \in W_{0, \Gamma\left(\overline{T_{\Phi}(\mathcal{X})}\right), \epsilon}$, então $\left|h^{\prime}(h)\right|<\epsilon, \forall h \in \Gamma\left(\overline{T_{\Phi}(\mathcal{X})}\right)$. Como $T_{\Phi}(\mathcal{X}) \subset \Gamma\left(\overline{T_{\Phi}(\mathcal{X})}\right)$, segue que $\left|h^{\prime}\left(T_{\Phi}(f)\right)\right|<\epsilon, \forall f \in \mathcal{X}$ e $T_{\Phi}^{\prime}\left(h^{\prime}\right) \in V_{0, \mathcal{X}, \epsilon}$. Portanto, $T_{\Phi}^{\prime}:\left(H_{b}(V, G)^{\prime}, \tau_{c}\right) \rightarrow\left(H_{b}(E, G)^{\prime}, \tau_{\beta}\right)$ é contínua.

Agora, seja $\mathcal{A} \subset H_{b}(V, G)^{\prime}$ um conjunto fortemente limitado, dado que $H_{b}(V, G)$ é tonelado, temos que $\mathcal{A} \subset H_{b}(V, G)^{\prime}$ é equicontínuo e consequentemente $\sigma\left(H_{b}(V, G)^{\prime}, H_{b}(V, G)\right)$ relativamente compacto. Pelo teorema de Banach-Dieudonné (ver [15, pg. 245]) segue que $\mathcal{A} \subset H_{b}(V, G)^{\prime}$ é $\tau_{c}$-relativamente compacto e como $T_{\Phi}^{\prime}$ é $\tau_{c}-\tau_{\beta}$ contínuo temos que $T_{\Phi}^{\prime}(\mathcal{A}) \subset H_{b}(E, G)^{\prime}$ é $\tau_{\beta}$-relativamente compacto. Portanto, $T_{\Phi}^{\prime}$ é compacto na topologia forte.

(b) $\Rightarrow(c)$ Seja $a \in G$ com $\|a\|=1$, pelo teorema de Hahn-Banach $\exists a^{\prime} \in G^{\prime}$ com $\left\|a^{\prime}\right\|=1$ tal que $a^{\prime}(a)=1$. Considerando as aplicações da proposição 4.1.2 temos que $\psi:=J_{a}^{\prime} \circ T_{\Phi}^{\prime} \circ \delta_{a^{\prime}}: V \rightarrow\left(E^{\prime \prime},\|\|\right)$ leva conjuntos $V$-limitados de $V$ em conjuntos relativamente compactos de $E^{\prime \prime}$.

De fato, seja $B \subset V$ um conjunto $V$-limitado, pela proposição 4.1 .2 (ii) temos que $\delta_{a^{\prime}}(B) \subset H_{b}(V, G)^{\prime}$ é fortemente limitado e por hipótese temos que $T_{\Phi}^{\prime} \circ \delta_{a^{\prime}}(B) \subset H_{b}(E, G)^{\prime}$ é $\tau_{\beta}$-relativamente compacto, pela proposição $4.1 .2(i)$ temos que $J_{a}^{\prime} \circ T_{\Phi}^{\prime} \circ \delta_{a^{\prime}}(B) \subset E^{\prime \prime}$ é relativamente compacto.

Por outro lado,

$$
\psi(y)\left(x^{\prime}\right)=\left(J_{a}^{\prime} \circ T_{\Phi}^{\prime} \circ \delta_{a^{\prime}}\right)(y)\left(x^{\prime}\right)=a^{\prime}\left(x^{\prime}(\Phi(y)) a\right)=x^{\prime}(\Phi(y)) a^{\prime}(a)=C(\Phi(y))\left(x^{\prime}\right)
$$

$\forall y \in V, \forall x^{\prime} \in E^{\prime}$ onde $C: E \rightarrow E^{\prime \prime}$ é a aplicação natural. Logo, $\psi(y)=C(\Phi(y)), \forall y \in V$, então $C \circ \Phi=\psi$. Como $\psi$ leva conjuntos $V$-limitados de $V$ em conjuntos relativamente compactos de $E^{\prime \prime}$ e a aplicação natural $C: E \rightarrow E^{\prime \prime}$ é uma isometria, temos que $\Phi$ é compacto.

Observamos que para $G=\mathbb{C}$ as afirmações do teorema 4.1 .4 são equivalentes e foi demonstrado por González-Gutiérrez em [11].

A proposição que demonstramos a seguir foi demonstrado em [13] por GalindoLourenço-Moraes para funções holomorfas de tipo limitado a valores complexos. Obser- 
vamos que tal resultado permanece válido quando trabalhamos com funções holomorfas de tipo limitado a valores vetoriais.

Proposição 4.1.5 Sejam E, F espaços de Banach e G uma álgebra de Banach. Sejam $U \subset E$ um conjunto aberto e equilibrado, $V \subset F$ um conjunto aberto e $T: H_{b}(U, G) \rightarrow$

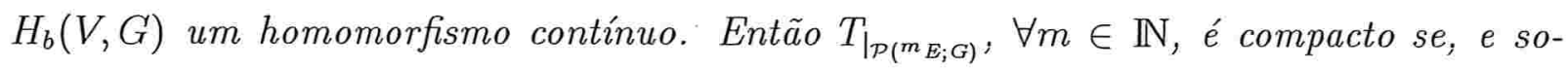
mente se, $T$ é compacto.

Demonstração. Se $T$ é compacto é claro que $T_{\left.\right|_{\mathcal{P}\left(m_{E ;} ;\right)}}, \forall m \in \mathbb{N}$, é compacto. Vamos agora supor que $T_{\left.\right|_{\mathcal{P}(m E ; G)}}, \forall m \in \mathbb{N}$, é compacto e mostrar que $T$ é compacto.

Sejam $\mathcal{X} \subset H_{b}(U, G)$ um conjunto limitado e $V_{0, B, \epsilon} \in \mathcal{B}_{H_{b}(V, G)}(0)$. Como $T$ é contínua existe $W_{0, D, \delta} \in \mathcal{B}_{H_{b}(U, G)}(0)$ tal que $T\left(W_{0, D, \delta}\right) \subset V_{0, B, \epsilon}$.

Dado que $\mathcal{X}$ é limitado, usando a desigualdade de Cauchy podemos achar $n \in \mathbb{N}$ tal que $\left\|f-\sum_{m=0}^{n} P^{m}(f)\right\|_{D} \leq \delta, \forall f \in \mathcal{X}$ (ver [17, proposição 1]), isto é:

$$
f-\sum_{m=0}^{n} P^{m}(f) \in W_{0, D, \delta}, \forall f \in \mathcal{X} .
$$

Por outro lado, $T(f)=T\left(f-\sum_{m=0}^{n} P^{m}(f)\right)+\sum_{m=0}^{n} T\left(P^{m}(f)\right), \forall f \in \mathcal{X}$ e consequentemente $T(f) \in V_{0, B, \epsilon}+\sum_{m=0}^{n} T\left(\mathcal{X}_{m}\right)$ onde $\mathcal{X}_{m}=\left\{P^{m}(f) / f \in \mathcal{X}\right\} \subset \mathcal{P}\left({ }^{m} E, G\right)$ é limitado (ver

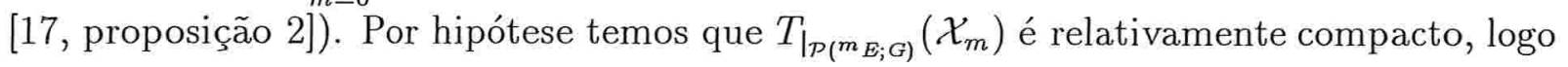
$\mathcal{K}:=\sum_{m=0}^{n} T\left(\mathcal{X}_{m}\right)$ também é relativamente compacto. Desta forma, temos que para cada vizinhança $V_{0, B, \epsilon} \in \mathcal{B}_{H_{b}(V, G)}(0)$, existe um conjunto relativamente compacto $\mathcal{K} \subset H_{b}(V, G)$ tal que $T(\mathcal{X}) \subset V_{0, B, \epsilon}+\mathcal{K}$. Segue de [6, 4(iii) pg. 5] que $T(\mathcal{X})$ é relativamente compacto, logo $T$ é compacto.

$\mathrm{Na}$ seguinte proposição vamos demonstrar que se operadores de composição $T_{\Phi}$ entre álgebras de funções holomorfas do tipo limitado é compacto então a função $\Phi$ é compacta.

Proposição 4.1.6 Sejam E, F espaços de Banach e G uma álgebra de Banach. Sejam $U \subset E$ um conjunto aberto e equilibrado, $V \subset F$ um conjunto aberto, $\Phi \in H_{b}(V, E)$ 
tal que $\Phi(V) \subset U$ e $T_{\Phi}: H_{b}(U, G) \rightarrow H_{b}(V, G)$ um operador de composição. Se $T_{\Phi}$ é compacto então $\Phi$ é compacta.

Demonstração. Vamos supor que $T_{\Phi}$ é compacto. Consideremos o operador de composição $A: H_{b}(E, G) \rightarrow H_{b}(V, G)$ dado por $A(f)=f \circ \Phi, \forall f \in H_{b}(E, G)$. Como por hipótese $T_{\Phi}$ é compacto e $H_{b}(E, G) \hookrightarrow H_{b}(U, G)$ segue que $A$ é compacto. Pelo teorema 4.1.4, temos que $\Phi: V \rightarrow E$ aplica conjuntos $V$-limitados $B$ em conjuntos $\Phi(B) \subset E$ relativamente compactos. Além disso, $\Phi(B)$ é $U$-limitado para cada conjunto $B V$-limitado, $\operatorname{logo} \Phi(B) \subset \overline{\Phi(B)} \subset U$ é relativamente compacto em $U$. Assim, $\Phi \in H_{b}(V, E)$ é compacta e $\Phi(V) \subset U$.

Denotamos por $\otimes_{\pi}^{j} E$ o produto tensorial $\otimes^{j} E=E \otimes \stackrel{(j)}{\cdots} \otimes E$ munido da norma tensorial projetiva $\pi$ dado por

$$
\pi(u)=\inf \left\{\sum_{i}\left\|x_{i, 1}\right\| \cdots\left\|x_{i, n}\right\|: u=\sum_{i} x_{i, 1} \otimes \cdots \otimes x_{i, n}\right\}
$$

onde o ínfimo é tomado sobre todas as representações finitas de $u$. $\otimes_{\pi}^{j} E$ é um espaço normado e denotamos por $\widehat{\otimes}_{\pi}^{j} E$ o seu completamento.

O seguinte lema será usado na proposição 4.1.8.

Lema 4.1.7 Sejam E, F e $G$ espaços de Banach tal que E tem a propriedade de DunfordPettis. Considere polinômios fracamente compactos $Q^{\ell_{r}} \in \mathcal{P}\left({ }^{\ell_{r}} F, E\right)$, com $1 \leq r \leq j$, para $j \in \mathbb{N}$ fixo. Então, $Q^{\left({ }^{\prime}\right)}: \mathcal{P}\left({ }^{j} E, G\right) \rightarrow \mathcal{P}\left({ }^{\ell_{1}+\cdots+\ell_{j}} F, G\right)$ dado por

$$
Q^{\left({ }^{\prime}\right)}\left(P^{j}\right)(y)=A^{j}\left(Q^{\ell_{1}}(y), \cdots, Q^{\ell_{j}}(y)\right), \forall y \in F, \forall P^{j}=\widehat{A}^{j} \in \mathcal{P}\left({ }^{j} E, G\right)
$$

é uma aplicação linear fracamente compacta.

Demonstração. Seja $Q: F \rightarrow \widehat{\otimes}_{\pi}^{j} E$ dado por $Q(y)=Q^{\ell_{1}}(y) \widehat{\otimes}_{\pi} \stackrel{(j)}{\cdots} \widehat{\otimes}_{\pi} Q^{\ell_{j}}(y), \forall y \in F$ um polinômio $\left(\ell_{1}+\cdots+\ell_{j}\right)$-homogêneo. Como cada $Q^{\ell_{r}}$ é fracamente compacto temos que $Q^{\ell_{r}}(B) \subset E$ é relativamente fracamente compacto para cada conjunto limitado $B$ de $F$. Por hipótese $E$ tem a propriedade de Dunford-Pettis, segue do teorema 16 de J. Diestel [5], que $Q(B)$ é relativamente fracamente compacto com

$$
Q(B)=Q^{\ell_{1}}(B) \widehat{\otimes}_{\pi} \cdot \stackrel{(j)}{\cdots} \widehat{\otimes}_{\pi} Q^{\ell_{j}}(B) \subset \widehat{\otimes}_{\pi}^{j} E
$$


para cada conjunto limitado $B$ de $F$. Portanto, $Q: F \rightarrow \widehat{\otimes}_{\pi}^{j} E$ é fracamente compacto.

Vamos agora a partir de $Q$ construir a aplicação linear $Q^{\left({ }^{\prime}\right)}$. Considere a aplicação linear $Q^{\prime}: \mathcal{L}\left(\widehat{\otimes}_{\pi}^{j} E, G\right) \rightarrow \mathcal{P}\left({ }^{\ell_{1}+\cdots+\ell_{j}} F, G\right)$ dada por $Q^{\prime}(A)=A \circ Q, \forall A \in \mathcal{L}\left(\widehat{\otimes}_{\pi}^{j} E, G\right)$. Seja $A_{Q} \in \mathcal{L}_{s}\left({ }^{\ell_{1}+\cdots+\ell_{j}} F, \widehat{\otimes}_{\pi}^{j} E\right)$ tal que $Q=\widehat{A_{Q}}$. Pela proposição 3 de A. Pelczynski [29] sabemos que $A_{Q}$ é $w$-compacta. Usando o fato que $\mathcal{L}\left({ }^{\ell_{1}+\cdots+\ell_{j}} F, \widehat{\otimes}_{\pi}^{j} E\right)$ é isometricamente isomorfo a $\mathcal{L}\left(\widehat{\otimes}_{\pi}^{\ell_{1}+\cdots+\ell_{j}} F, \widehat{\otimes}_{\pi}^{j} E\right)$, existe uma aplicação linear $L_{Q}$ associado ao $A_{Q}$. Por Pelczynski [29] sabemos que $L_{Q}$ é $w$-compacto.

Consideremos a aplicação linear $L_{Q}^{t}: \mathcal{L}\left(\widehat{\otimes}_{\pi}^{j} E, G\right) \rightarrow \mathcal{L}\left(\widehat{\otimes}_{\pi}^{\ell_{1}+\cdots+\ell_{j}} F, G\right)$ dada por $L_{Q}^{t}(A)=A \circ L_{Q}, \forall A \in \mathcal{L}\left(\widehat{\otimes}_{\pi}^{j} E, G\right)$. Agora do teorema 9.31 de [10, pg. 623] seque que $L_{Q}^{t}$ é $w$-compacto.

Seja $J: \mathcal{P}\left({ }^{\ell_{1}+\cdots+\ell_{j}} F, G\right) \rightarrow \mathcal{L}_{s}\left({ }^{\ell_{1}+\cdots+\ell_{j}} F, G\right)$ a isometria natural dada por $J(Q)=A_{Q}, \forall Q \in \mathcal{P}\left({ }^{\ell_{1}+\cdots+\ell_{j}} F, G\right)$. Então $J \circ Q^{\prime}: \mathcal{L}\left(\widehat{\otimes}_{\pi}^{j} E, G\right) \rightarrow \mathcal{L}_{s}\left({ }^{\ell_{1}+\cdots+\ell_{j}} F, G\right)$ é dado por $J \circ Q^{\prime}(A)=A \circ A_{Q}, \forall A \in \mathcal{L}\left(\widehat{\otimes}_{\pi}^{j} E, G\right)$, pelo isomorfismo acima citado temos que $J \circ Q^{\prime}(A)=L_{Q}^{t}(A), \forall A \in \mathcal{L}\left(\widehat{\otimes}_{\pi}^{j} E, G\right)$. Consequentemente $Q^{\prime}=J^{-1} \circ L_{Q}^{t}$ é $w$-compacto.

Assim, por uma identificação e abuso de notação temos que a aplicação linear $Q^{\left({ }^{\prime}\right)}=Q_{\left.\right|_{\mathcal{P}\left({ }^{j} E, G\right)} ^{\prime}}^{\prime}: \mathcal{P}\left({ }^{j} E, G\right) \rightarrow \mathcal{P}\left({ }^{\ell_{1}+\cdots+\ell_{j}} F, G\right)$ é w-compacto.

Na seguinte proposição vamos demonstrar uma caracterização para que operadores de composição entre álgebras de funções holomorfas de tipo limitada sejam fracamente compactos. Para tal observamos que $H_{b}(E, G)^{\prime}$ e $\left(H_{b}(E, G)^{\prime}, \tau_{\beta}\right)^{\prime}$ formam um par dual.

Proposição 4.1.8 Sejam E, F e $G$ espaços de Banach, tal que E tem a propriedade de Dunford-Pettis. Sejam $V \subset F$ subconjunto aberto e equilibrado, $\Phi \in H_{b}(V, E)$ e $T_{\Phi}: H_{b}(E, G) \rightarrow H_{b}(V, G)$ um operador de composição. Então as seguintes afirmações são equivalentes.

(a) $T_{\Phi}$ é fracamente compacto;

(b) o adjunto $T_{\Phi}^{\prime}: H_{b}(V, G)^{\prime} \rightarrow H_{b}(E, G)^{\prime}$ aplica conjuntos $\tau_{\beta}$-limitados em conjuntos $\sigma\left(H_{b}(E, G)^{\prime},\left(H_{b}(E, G)^{\prime}, \tau_{\beta}\right)^{\prime}\right)$-relativamente compactos; 
(c) $\Phi$ é uma aplicação fracamente compacta.

Demonstração. Para demonstrar $(a) \Rightarrow(b)$, vamos primeiramente mostrar que $T_{\Phi}^{\prime}:\left(H_{b}(V, G)^{\prime}, \tau_{\mu}\right) \rightarrow\left(H_{b}(E, G)^{\prime}, \tau_{\beta}\right)$ é contínua onde $\tau_{\mu}$ é a topologia de Mackey $\mu\left(H_{b}(V, G)^{\prime}, H_{b}(V, G)\right)$ e $\tau_{\beta}$ é a topologia forte.

Para tal, seja $V_{0, \mathcal{X}, \epsilon}=\left\{f^{\prime} \in H_{b}(E, G)^{\prime} /\left\|f^{\prime}\right\|_{\mathcal{X}}<\epsilon\right\} \in \mathcal{B}_{\left(H_{b}(E, G)^{\prime}, \tau_{\beta}\right)}(0)$, onde $\mathcal{X} \subset H_{b}(E, G)$ é limitado. Como por hipótese $T_{\Phi}: H_{b}(E, G) \rightarrow H_{b}(V, G)$ é fracamente compacto, segue que $T_{\Phi}(\mathcal{X})$ é relativamente fracamente compacto. Assim, $\Gamma\left(\overline{T_{\Phi}(\mathcal{X})}\right)$, a envoltoria absolutamente convexa, é fracamente compacto, uma vez que $H_{b}(V, G)$ é Fréchet.

Considere $W_{0, \Gamma\left(\overline{T_{\Phi}(\mathcal{X})}\right), \epsilon}=\left\{h^{\prime} \in H_{b}(V, G)^{\prime} /\left\|h^{\prime}\right\|_{\Gamma\left(\overline{T_{\Phi}(\mathcal{X})}\right)}<\epsilon\right\} \in \mathcal{B}_{\left(H_{b}(V, G)^{\prime}, \tau_{\mu}\right)}(0)$, então $T_{\Phi}^{\prime}\left(W_{0, \Gamma\left(\overline{T_{\Phi}(\mathcal{X})}\right), \epsilon}\right) \subset V_{0, \mathcal{X}, \epsilon}$, uma vez que para cada $h^{\prime} \in W_{0, \Gamma\left(\overline{T_{\Phi}(\mathcal{X})}\right), \epsilon}$, temos que $\left|h^{\prime}(h)\right|<\epsilon, \forall h \in \Gamma\left(\overline{T_{\Phi}(\mathcal{X})}\right)$ e como $T_{\Phi}(\mathcal{X}) \subset \Gamma\left(\overline{T_{\Phi}(\mathcal{X})}\right)$, temos que $\left|h^{\prime}\left(T_{\Phi}(f)\right)\right|<\epsilon, \forall f \in \mathcal{X}$ e consequentemente $T_{\Phi}^{\prime}\left(h^{\prime}\right) \in V_{0, \mathcal{X}, \epsilon}$ e $T_{\Phi}^{\prime}:\left(H_{b}(V, G)^{\prime}, \tau_{\mu}\right) \rightarrow\left(H_{b}(E, G)^{\prime}, \tau_{\beta}\right)$ é contínua.

Agora afirmamos que $T_{\Phi}^{\prime}:\left(H_{b}(V, G)^{\prime}, w^{*}\right) \rightarrow\left(H_{b}(E, G)^{\prime}, \sigma\left(H_{b}(E, G)^{\prime},\left(H_{b}(E, G)^{\prime}, \tau_{\beta}\right)^{\prime}\right)\right)$ é contínua onde $w^{*}$ é a topologia fraca estrela.

De fato, seja $V_{0, f_{1}^{\prime \prime}, \cdots, f_{k}^{\prime \prime}, \epsilon} \in \mathcal{B}_{\left(H_{b}(E, G)^{\prime}, \sigma\left(H_{b}(E, G)^{\prime},\left(H_{b}(E, G)^{\prime}, \tau_{\beta}\right)^{\prime}\right)\right)}(0)$, onde $f_{i}^{\prime \prime} \in\left(H_{b}(E, G)^{\prime}, \tau_{\beta}\right)^{\prime}$, $i=1,2, \cdots, k$. Pela parte anterior, para o adjunto de $T_{\Phi}^{\prime}$ temos que $T_{\Phi}^{\prime \prime}\left(f_{i}^{\prime \prime}\right) \in\left(H_{b}(V, G)^{\prime}, \tau_{\mu}\right)^{\prime}$ $\operatorname{logo} \exists f_{i} \in H_{b}(V, G)$ tal que $T_{\Phi}^{\prime \prime}\left(f_{i}^{\prime \prime}\right)\left(f^{\prime}\right)=f^{\prime}\left(f_{i}\right), \forall f^{\prime} \in H_{b}(V, G)^{\prime}, i=1,2, \cdots, k$.

Consideremos $W_{0, f_{1}, \cdots, f_{k}, \epsilon} \in \mathcal{B}_{\left(H_{b}(V, G)^{\prime}, w^{*}\right)}(0)$, e mostremos que

$$
T_{\Phi}^{\prime}\left(W_{0, f_{1}, \cdots, f_{k}, \epsilon}\right) \subset V_{0, f_{1}^{\prime \prime}, \cdots, f_{k}^{\prime \prime}, \epsilon}
$$

Seja $f^{\prime} \in W_{0, f_{1}, \cdots, f_{k}, \epsilon}, \operatorname{assim}\left|f^{\prime}\left(f_{i}\right)\right|<\epsilon, i=1,2, \cdots, k \operatorname{logo}\left|T_{\Phi}^{\prime \prime}\left(f_{i}^{\prime \prime}\right)\left(f^{\prime}\right)\right|<\epsilon, i=$ $1,2, \cdots, k$, então $\left|f_{i}^{\prime \prime}\left(T_{\Phi}^{\prime}\left(f^{\prime}\right)\right)\right|<\epsilon, i=1,2, \cdots, k$, consequentemente $T_{\Phi}^{\prime}\left(f^{\prime}\right) \in V_{0, f_{1}^{\prime \prime}, \cdots, f_{k}^{\prime \prime}, \epsilon}$. Portanto, $T_{\Phi}^{\prime}$ é $w^{*}-\sigma\left(H_{b}(E, G)^{\prime},\left(H_{b}(E, G)^{\prime}, \tau_{\beta}\right)^{\prime}\right)$ contínua na origem e consequentemente contínua.

Agora, seja $\mathcal{A} \subset H_{b}(V, G)^{\prime}$ um conjunto fortemente limitado, dado que $H_{b}(V, G)$ é tonelado, temos que $\mathcal{A} \subset H_{b}(V, G)^{\prime}$ é relativamente $w^{*}$-compacto e como $T_{\Phi}^{\prime}$ é $w^{*}-$ $\sigma\left(H_{b}(E, G)^{\prime},\left(H_{b}(E, G)^{\prime}, \tau_{\beta}\right)^{\prime}\right)$ contínua temos que $T_{\Phi}^{\prime}(\mathcal{A})$ é $\sigma\left(H_{b}(E, G)^{\prime},\left(H_{b}(E, G)^{\prime}, \tau_{\beta}\right)^{\prime}\right)$ - 
relativamente compacto.

Portanto, $T_{\Phi}^{\prime}$ aplica $\tau_{\beta}$-limitados em $\sigma\left(H_{b}(E, G)^{\prime},\left(H_{b}(E, G)^{\prime}, \tau_{\beta}\right)^{\prime}\right)$-relativamente compactos.

$(b) \Rightarrow(c)$ Seja $a \in G$ com $\|a\|=1$, pelo teorema de Hahn-Banach $\exists a^{\prime} \in G^{\prime}$ com $\left\|a^{\prime}\right\|=1$ tal que $a^{\prime}(a)=1$. Consideremos as seguintes aplicações definidas na proposição 4.1.2, $J_{a}:\left(E^{\prime},\|\|\right) \rightarrow H_{b}(E, G)$ tal que $J_{a}\left(x^{\prime}\right)(x)=x^{\prime}(x) a, \forall x^{\prime} \in E^{\prime}, \forall x \in E \quad$ e $\delta_{a^{\prime}}: V \rightarrow H_{b}(V, G)^{\prime}$ tal que $\delta_{a^{\prime}}(y)(h)=a^{\prime}(h(y)), \forall y \in V, \forall h \in H_{b}(V, G)$.

Primeiramente vamos mostrar que $J_{a}^{\prime}:\left(H_{b}(E, G)^{\prime}, \sigma\left(H_{b}(E, G)^{\prime},\left(H_{b}(E, G)^{\prime}, \tau_{\beta}\right)^{\prime}\right)\right) \rightarrow$ $\left(E^{\prime \prime}, w^{*}\right)$ é contínua na origem.

Seja $V_{0, x_{1}^{\prime}, \cdots, x_{r}^{\prime}, \epsilon} \in \mathcal{B}_{\left(E^{\prime \prime}, w^{*}\right)}(0)$, para cada $i=1,2, \cdots, r$ consideremos as aplicações $x_{i}^{\prime} \otimes a \in H_{b}(E, G)$ onde $x_{i}^{\prime} \otimes a(x)=x_{i}^{\prime}(x) a, \forall x \in E$ e as aplicações lineares contínuas $f_{i}^{\prime \prime}:\left(H_{b}(E, G)^{\prime}, \tau_{\beta}\right) \rightarrow \mathbb{C}$ dadas por $f_{i}^{\prime \prime}\left(f^{\prime}\right)=f^{\prime}\left(x_{i}^{\prime} \otimes a\right), \forall f^{\prime} \in H_{b}(E, G)^{\prime}$.

Agora, sejam $W_{0, f_{1}^{\prime \prime}, \cdots, f_{r}^{\prime \prime}, \epsilon} \in \mathcal{B}_{\left(H_{b}(E, G)^{\prime}, \sigma\left(H_{b}(E, G)^{\prime},\left(H_{b}(E, G)^{\prime}, \tau_{\beta}\right)^{\prime}\right)\right)}(0)$ e $f^{\prime} \in W_{0, f_{1}^{\prime \prime}, \cdots, f_{r}^{\prime \prime}, \epsilon} \operatorname{logo}$ $\left|f_{i}^{\prime \prime}\left(f^{\prime}\right)\right|<\epsilon, i=1, \cdots, r$, então $\left|J_{a}^{\prime}\left(f^{\prime}\right)\left(x_{i}^{\prime}\right)\right|<\epsilon, i=1, \cdots, r$ portanto $J_{a}^{\prime}\left(f^{\prime}\right) \in V_{0, x_{1}^{\prime}, \cdots, x_{r}^{\prime}, \epsilon}$. Consequentemente, $J_{a}^{\prime}$ é $\sigma\left(H_{b}(E, G)^{\prime},\left(H_{b}(E, G)^{\prime}, \tau_{\beta}\right)^{\prime}\right)-w^{*}$ contínua.

Assim a aplicação $\psi: V \rightarrow\left(E^{\prime \prime}, w^{*}\right)$ dada por $\psi=J_{a}^{\prime} \circ T_{\Phi}^{\prime} \circ \delta_{a^{\prime}}$, leva conjuntos $V$ limitados de $V$ em conjuntos $w^{*}$-relativamente compactos de $E^{\prime \prime}$, desde que para cada $B \subset$ $V$ conjunto $V$-limitado, temos pela proposição $4.1 .2(i i)$ que $\delta_{a^{\prime}}(B) \subset H_{b}(V, G)^{\prime}$ é fortemente limitado e assim por hipótese $T_{\Phi}^{\prime} \circ \delta_{a^{\prime}}(B) \subset H_{b}(E, G)^{\prime}$ é $\sigma\left(H_{b}(E, G)^{\prime},\left(H_{b}(E, G)^{\prime}, \tau_{\beta}\right)^{\prime}\right)^{-}$ relativamente compacto. Agora pela parte anterior, segue que $J_{a}^{\prime} \circ T_{\Phi}^{\prime} \circ \delta_{a^{\prime}}(B) \subset E^{\prime \prime}$ é $w^{*}$-relativamente compacto. Além disso,

$\psi(y)\left(x^{\prime}\right)=\left(J_{a}^{\prime} \circ T^{\prime} \circ \delta_{a^{\prime}}\right)(y)\left(x^{\prime}\right)=a^{\prime}\left(x^{\prime}(\Phi(y)) a\right)=x^{\prime}(\Phi(y))=C(\Phi(y))\left(x^{\prime}\right), \forall y \in V, \forall x^{\prime} \in E^{\prime}$.

Agora, seja $\left(y_{n}\right)_{n \in \mathbb{N}} \subset V$ uma seqüência limitada e como $\psi$ é $w^{*}$-relativamente compacto existem uma subseqüência $\left(y_{n_{k}}\right)_{k}$ de $\left(y_{n}\right)$ e $x^{\prime \prime} \in E^{\prime \prime}$ tal que

$$
x^{\prime}\left(\Phi\left(y_{n_{k}}\right)\right) \rightarrow x^{\prime \prime}\left(x^{\prime}\right), \forall x^{\prime} \in E^{\prime}
$$

Consequentemente, $\left(x^{\prime}\left(\Phi\left(y_{n_{k}}\right)\right)\right)_{k}$ converge em $\mathbb{C}$ para cada $x^{\prime} \in E^{\prime}$, logo $\Phi(V)$ é fracamente relativamente compacto em $E$ e portanto, $\Phi$ é fracamente compacto. 
$(c) \Rightarrow(a)$ Seja $\left(f_{n}\right)_{n} \subset H_{b}(E, G)$ uma seqüência limitada. Vamos mostrar que. $\left(T_{\Phi}\left(f_{n}\right)\right)_{n} \subset H_{b}(V, G)$ admite uma subseqüência $w$-convergente, isto é, $\left(f_{n} \circ \Phi\right)_{n}$ admite subseqüência $w$-convergente. Para cada $n \in \mathbb{N}$ a série de Taylor de $f_{n} \circ \Phi$ em $0 \in V$ é dado por $f_{n} \circ \Phi(z)=\sum_{k=0}^{\infty} \frac{\widehat{d}^{k}\left(f_{n} \circ \Phi(0)\right)}{k !}(z)$.

Agora, para cada $k \in \mathbb{N}$ temos que

$$
\frac{\widehat{d^{k}}\left(f_{n} \circ \Phi(0)\right)}{k !}=\sum_{j=1}^{k} \sum_{\substack{\ell_{1}+\cdots+\ell_{j}=k \\\left(\ell_{1}, \cdots, \ell_{j}\right) \in \mathbb{N}^{j}}} \frac{d^{j} f_{n}(\Phi(0))}{j !}\left(\frac{\widehat{d}^{\ell_{1}} \Phi(0)}{\ell_{1} !}, \cdots, \frac{\widehat{d}^{\ell_{j}} \Phi(0)}{\ell_{j} !}\right), \forall n \in \mathbb{N}
$$

(ver [24, proposição 6, pg. 216] ou [31, pg. 419]) onde cada $\frac{d^{j} f_{n}(\Phi(0))}{j !}$ denota aplicação $j$-linear associado ao polinômio $\frac{\widehat{d}^{j} f_{n}(\Phi(0))}{j !} \in \mathcal{P}\left({ }^{j} E, G\right)$.

Como $\Phi: V \rightarrow E$ é fracamente compacto, por Ryan [30, teorema 3.2] segue que $\frac{\widehat{d}^{\ell} \Phi(0)}{\ell !} \in \mathcal{P}\left({ }^{\ell} F, E\right)$ é um polinômio fracamente compacto para cada $\ell \in \mathbb{N}$. Como a seqüência $\left(\frac{\widehat{d}^{j} f_{n}(\Phi(0))}{j !}\right)_{n \in \mathbb{N}} \subset \mathcal{P}\left({ }^{j} E, G\right)$ é limitada, segue do lema 4.1 .7 que a seqüência

$$
\left(Q^{\left({ }^{\prime}\right)}\left(\frac{\widehat{d}^{j} f_{n}(\Phi(0))}{j !}\right)\right)_{n \in \mathbb{N}}=\left(\frac{d^{j} f_{n}(\Phi(0))}{j !}\left(\frac{\widehat{d}^{\ell_{1}} \Phi(0)}{\ell_{1} !}, \cdots, \frac{\widehat{d}^{\ell_{j}} \Phi(0)}{\ell_{j} !}\right)\right)_{n \in \mathbb{N}}
$$

tem uma subseqüência fracamente convergente para cada $j \in \mathbb{N}$ tal que $\ell_{1}+\cdots+\ell_{j}=k$ e $\left(\ell_{1}, \cdots, \ell_{j}\right) \in \mathbb{N}^{j}$. Assim, a soma finita de (4.2) tem uma subseqüência fracamente convergente, que ainda denotaremos por $\left(\frac{\widehat{d}^{k}\left(f_{n} \circ \Phi(0)\right)}{k !}\right)_{n \in \mathbb{N}}$.

Agora, para $k=1$ podemos obter um subconjunto infinito $\mathbb{N}_{1} \subset \mathbb{N}$ tal que existe o limite $Q_{1}=\lim _{n \in \mathbb{N}_{1}} \frac{\widehat{d}^{1}\left(f_{n} \circ \Phi(0)\right)}{1 !}$ na topologia fraca. Como $\left(\frac{\widehat{d}^{2}\left(f_{n} \circ \Phi(0)\right)}{2 !}\right)_{n \in \mathbb{N}}$ é fracamente convergente podemos achar um subconjunto infinito $\mathbb{N}_{2} \subset \mathbb{N}_{1}$ tal que existe o limite $Q_{2}=\lim _{n \in \mathbb{N}_{2}} \frac{\widehat{d}^{2}\left(f_{n} \circ \Phi(0)\right)}{2 !}$ na topologia fraca. Prosseguindo analogamente, conseguimos, para cada $i \in \mathbb{N}$, um subconjunto infinito $\mathbb{N}_{i} \subset \mathbb{N}$, de tal forma que $\mathbb{N}_{1} \supset \mathbb{N}_{2} \supset \cdots \supset \mathbb{N}_{i} \supset \cdots$ e, para cada $i$, existe o limite $Q_{i}=\lim _{n \in \mathbb{N}_{i}} \frac{\widehat{d^{\imath}}\left(f_{n} \circ \Phi(0)\right)}{i !}$ na topologia fraca. Definimos então um subconjunto infinito $\mathbb{N}^{*} \subset \mathbb{N}$ tomando como $i$-ésimo elemento de $\mathbb{N}^{*}$ o elemento $i$ ésimo de $\mathbb{N}_{i}$. Desta maneira, para cada $i \in \mathbb{N}$, a seqüência $\left(\frac{\widehat{d}^{i}\left(f_{n} \circ \Phi(0)\right)}{i !}\right)_{n \in \mathbb{N}^{*}}$ é, a partir do seu $i$-ésimo elemento, uma subseqüência de $\left(\frac{\widehat{d}^{\imath}\left(f_{n} \circ \Phi(0)\right)}{i !}\right)_{n \in \mathbb{N}_{i}}$ e, portanto, fracamente convergente. Isto prova que a subseqüência $\left(\frac{\widehat{d}^{\natural}\left(f_{n} \circ \Phi(0)\right)}{i !}\right)_{n \in \mathbb{N}^{*}}$ é fracamente convergente para todo $i \in \mathbb{N}$. 
Segue, do lema 4.1 de [12], que $\left(T_{\Phi}\left(f_{n}\right)\right)_{n}=\left(f_{n} \circ \phi\right)_{n}$ é fracamente convergente.

Observamos que o lema 4.1.7 e a proposição 4.1.8 foram demonstrados por GonzálezGutiérrez em [12] para o caso $G=\mathbb{C}$.

\subsection{Operadores de composição entre álgebras de ger- mes holomorfos a valores vetoriais}

Nesta seção apresentamos uma caracterização de quando os homomorfismos contínuos entre álgebras de germes de funções holomorfas a valores em uma álgebra de Banach é um operador de composição. A demonstração de tal resultado será feita utilizando uma fatoração através de álgebras de funções holomorfas do tipo limitado definidas em abertos de um espaço de Banach a valores em uma álgebra de Banach. Isto é o que faremos na proposição 4.2 .2 .

De forma análoga a notação 1.2 .5 consideramos as seguintes notações para a álgebra $\left(\mathcal{H}(K, G), \tau_{w}\right)$.

Notações 4.2.1 Sejam E, $F$ e $G$ espaços de Banach, $K \subset E$ subconjunto compacto.

(a) Denotamos por $\left(\mathcal{H}(K, G), \tau_{w}\right)$ o limite indutivo com relação as inclusões contínuas $\mathcal{J}_{U}^{K}:\left(H(U, G), \tau_{w}\right) \hookrightarrow \mathcal{H}(K, G)$ com $U \supset K$ aberto e para cada $f \in H(U, G)$ denotaremos por $[f]=\mathcal{J}_{U}^{K}(f)$ a classe de equivalência determinada por $f$. E escrevemos $\left(\mathcal{H}(K, G), \tau_{w}\right)=\lim _{\overrightarrow{U \supset K}}\left(H(U, G), \tau_{w}\right)$ como o espaço de germes de funções holomorfas no compacto $K \subset E$ com a topologia de Nachbin $\tau_{w}$.

O espaço $\left(\mathcal{H}(K, G), \tau_{w}\right)$ é localmente convexo, Hausdorff $e$

$$
\left(\mathcal{H}(K, G), \tau_{w}\right)=\lim _{\overline{n \in \mathbb{N}}} H^{\infty}\left(U_{n}, G\right),
$$

onde $U_{n}=K+B_{\frac{1}{n}}(0)$ é subconjunto aberto de $E$ (ver [4,pg. 346, 348]). 
Observamos que, se $K$ é convexo e/ou equilibrado então $U_{n}$ é convexo e/ou equilibrado.

Dado que $H^{\infty}\left(U_{n}, G\right) \hookrightarrow H_{b}\left(U_{n}, G\right) \hookrightarrow\left(\mathcal{H}(K, G), \tau_{w}\right)$ temos que $\left(\mathcal{H}(K, G), \tau_{w}\right)=$ $\lim _{n \in \mathbb{N}} H_{b}\left(U_{n}, G\right)$, onde $\mathcal{J}_{n}^{K}: H_{b}\left(U_{n}, G\right) \hookrightarrow \mathcal{H}(K, G)$ é a inclusão natural e denotamos por $[f]=\mathcal{J}_{n}^{K}(f), \forall f \in H_{b}\left(U_{n}, G\right)$ a classe de equivalência determinada por $f$.

(b) Se $G$ é uma álgebra de Banach, então $\left(\mathcal{H}(K, G), \tau_{w}\right)$ é uma álgebra localmente $m$ convexa e a demonstração pode ser feita de modo análogo a demonstração dado por J. Mujica em [20, teorema 7.1] quando $G=\mathbb{C}$.

Proposição 4.2.2 Sejam E, $F$ espaços de Banach e G uma álgebra de Banach. Sejam $K \subset E, L \subset F$ conjuntos compactos e $A:\left(\mathcal{H}(K, G), \tau_{w}\right) \rightarrow\left(\mathcal{H}(L, G), \tau_{w}\right)$ um homomorfismo contínuo. Então para cada aberto $U_{n} \supset K$ existem um aberto $V_{m_{n}} \supset L$ e um homomorfismo contínuo $\widetilde{A}_{n}: H_{b}\left(U_{n}, G\right) \rightarrow H_{b}\left(V_{m_{n}}, G\right)$ tal que $\mathcal{J}_{m_{n}}^{L} \circ \widetilde{A}_{n}=A \circ \mathcal{J}_{n}^{K}$.

Demonstração. Sejam $U_{n} \supset K$ um conjunto aberto e o homomorfismo contínuo $A \circ \mathcal{J}_{n}^{K}: H_{b}\left(U_{n}, G\right) \rightarrow \mathcal{H}(K, G)$. Segue do teorema 1.2.11 de Grothendieck, que existem um conjunto aberto $V_{m_{l_{n}}} \supset L$ e uma aplicação linear contínua

$$
\overline{A \circ \mathcal{J}_{n}^{K}}: H_{b}\left(U_{n}, G\right) \rightarrow H_{b}\left(V_{m_{l_{n}}}, G\right) \text { tal que } \mathcal{J}_{m_{l_{n}}}^{L} \circ \overline{A \circ \mathcal{J}_{n}^{K}}=A \circ \mathcal{J}_{n}^{K} \text {. }
$$

Isto é, o seginte diagrama é comutativo.

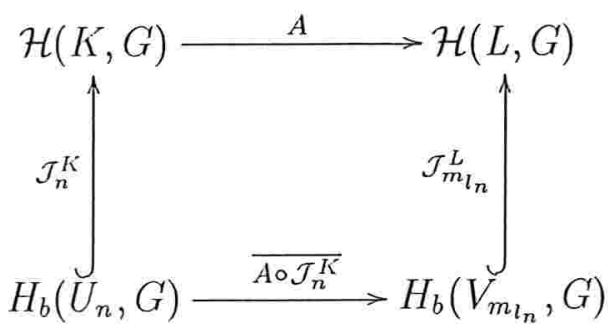

Observamos que cada $\mathcal{J}_{n}^{K}$ e $\mathcal{J}_{m}^{L}$ são homomorfismos contínuos, e como $A$ é homomorfismo temos:

$\left[\overline{A \circ \mathcal{J}_{n}^{K}}(f \cdot g)\right]=A\left(\mathcal{J}_{n}^{K}(f) \cdot \mathcal{J}_{n}^{K}(g)\right)=\left[\overline{A \circ \mathcal{J}_{n}^{K}}(f)\right]\left[\overline{A \circ \mathcal{J}_{n}^{K}}(g)\right]=\left[\overline{A \circ \mathcal{J}_{n}^{K}}(f) \cdot \overline{A \circ \mathcal{J}_{n}^{K}}(g)\right]$ 
então, $\overline{A \circ \mathcal{J}_{n}^{K}}(f \cdot g) \sim \overline{A \circ \mathcal{J}_{n}^{K}}(f) \cdot \overline{A \circ \mathcal{J}_{n}^{K}}(g), \forall f, g \in H_{b}\left(U_{n}, G\right)$.

Consequentemente, existe um conjunto aberto $V_{m_{n}} \subset V_{m_{l_{n}}}$ tal que

$$
\overline{A \circ \mathcal{J}_{n}^{K}}(f \cdot g)_{\left.\right|_{v_{m_{n}}}}=\left(\overline{A \circ \mathcal{J}_{n}^{K}}(f) \cdot \overline{A \circ \mathcal{J}_{n}^{K}}(g)\right)_{\left.\right|_{v_{m_{n}}}} \mathrm{e} H_{b}\left(V_{m_{l_{n}}}, G\right) \stackrel{i_{m_{l_{n}}}^{L}}{\hookrightarrow} H_{b}\left(V_{m_{n}}, G\right)
$$

Assim, $\mathcal{J}_{m_{n}}^{L} \circ i_{m_{l_{n}}}^{L}=\mathcal{J}_{m_{l_{n}}}^{L}$ e $\left(i_{m_{l_{n}}}^{L} \circ \overline{A \circ \mathcal{J}_{n}^{K}}\right)(f \cdot g)=\left(i_{m_{l_{n}}}^{L} \circ \overline{A \circ \mathcal{J}_{n}^{K}}\right)(f) \cdot\left(i_{m_{l_{n}}}^{L} \circ \overline{A \circ \mathcal{J}_{n}^{K}}\right)(g)$. A partir daí definimos o homomorfismo contínuo $\widetilde{A}_{n}: H_{b}\left(U_{n}, G\right) \rightarrow H_{b}\left(V_{m_{n}}, G\right)$ por $\widetilde{A}_{n}=i_{m_{l_{n}}}^{L} \circ \overline{A \circ \mathcal{I}_{n}^{K}}$ tal que $A \circ \mathcal{J}_{n}^{K}=\mathcal{J}_{m_{l_{n}}}^{L} \circ \overline{A \circ \mathcal{J}_{n}^{K}}=\mathcal{J}_{m_{n}}^{L} \circ i_{m_{l_{n}}}^{L} \circ \overline{A \circ \mathcal{J}_{n}^{K}}=\mathcal{J}_{m_{n}}^{L} \circ \widetilde{A}_{n}$.

Portanto, para cada aberto $U_{n} \supset K$ existem um aberto $V_{m_{n}} \supset L$ e um homomorfismo contínuo $\widetilde{A}_{n}: H_{b}\left(U_{n}, G\right) \rightarrow H_{b}\left(V_{m_{n}}, G\right)$ tal que o seguinte diagrama é comutativo.

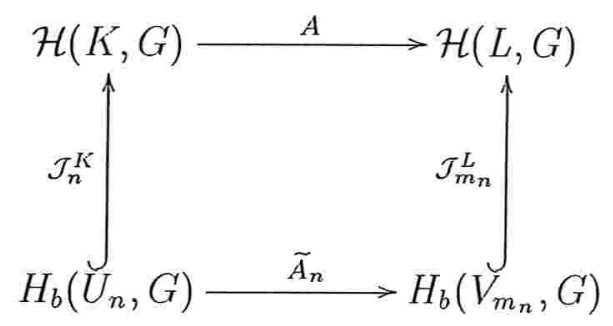

Isto é, $\mathcal{J}_{m_{n}}^{L} \circ \widetilde{A}_{n}=A \circ \mathcal{J}_{n}^{K}$.

Teorema 4.2.3 Sejam E, F espaços de Banach e $G$ uma álgebra de Banach. Sejam $K \subset$ $E, L \subset F$ conjuntos compactos e $A:\left(\mathcal{H}(K, G), \tau_{w}\right) \rightarrow\left(\mathcal{H}(L, G), \tau_{w}\right)$ um homomorfismo contínuo. Então A é um operador de composição se, e somente se, para cada aberto $U_{n} \supset K$ existem um aberto $V_{m_{n}} \supset L$ e um operador de composição $\widetilde{A}_{n}: H_{b}\left(U_{n}, G\right) \rightarrow$ $H_{b}\left(V_{m_{n}}, G\right)$ tal que $\mathcal{J}_{m_{n}}^{L} \circ \widetilde{A}_{n}=A \circ \mathcal{J}_{n}^{K}$.

Demonstração. Usaremos as notações de 4.2 .1 para os limites $\left(\mathcal{H}(K, G), \tau_{w}\right)=$ $\lim _{\substack{n \in \mathbb{N}\\}} H_{b}\left(U_{n}, G\right)$ e $\left(\mathcal{H}(L, G), \tau_{w}\right)=\lim _{n \in \mathbb{N}} H_{b}\left(V_{n}, G\right)$.

Vamos primeiramente supor que para cada aberto $U_{n} \supset K$ existem um aberto $V_{m_{n}} \supset$ $L$ e um operador de composição $\widetilde{A}_{n}: H_{b}\left(U_{n}, G\right) \rightarrow H_{b}\left(V_{m_{n}}, G\right)$ tal que $\mathcal{J}_{m_{n}}^{L} \circ \widetilde{A}_{n}=A \circ \mathcal{J}_{n}^{K}$.

Sem perda de generalidade podemos supor que $V_{m_{1}} \supset V_{m_{2}} \supset V_{m_{3}} \supset \cdots$. 
Vamos demonstrar que $A$ é um operador de composição.

Como cada $\widetilde{A}_{n}: H_{b}\left(U_{n}, G\right) \rightarrow H_{b}\left(V_{m_{n}}, G\right)$ é um operador de composição, segue que existe uma aplicação $\Phi_{n} \in H_{b}\left(V_{m_{n}}, E\right) \operatorname{com} \Phi_{n}\left(V_{m_{n}}\right) \subset U_{n}$ tal que $\widetilde{A}_{n}(f)=f \circ \Phi_{n}$, $\forall f \in H_{b}\left(U_{n}, G\right)$. Assim,

$$
\begin{aligned}
A([f]) & =\left(A \circ \mathcal{J}_{n}^{K}\right)(f)=\left(\mathcal{J}_{m_{n}}^{L} \circ \widetilde{A}_{n}\right)(f) \\
& =\left[\widetilde{A}_{n}(f)\right]=\left[f \circ \Phi_{n}\right], \forall f \in H_{b}\left(U_{n}, G\right), \forall n \in \mathbb{N}
\end{aligned}
$$

Agora, afirmamos que existem um conjunto aberto $V_{m_{1}} \supset L$ e $\Phi \in H_{b}\left(V_{m_{1}}, E\right)$ tal que $\Phi(L) \subset K$, onde $A([f])=[f \circ \Phi], \forall f \in H_{b}\left(U_{n}, G\right), \forall n \in \mathbb{N}$.

De fato, considerando o conjunto $V_{m_{1}}$ acima podemos definir uma aplicação $\Phi: V_{m_{1}} \rightarrow E$ por $\Phi(y)=\Phi_{1}(y), \forall y \in V_{m_{1}}$.

Mostraremos que $\Phi=\Phi_{n}$ sobre cada $V_{m_{n}}$. Seja $a \in G$ com $\|a\|=1$ e consideramos a inclusão $\left(E^{\prime},\|\|\right) \stackrel{\varrho_{a}^{n}}{\leftrightarrow} H_{b}\left(U_{n}, G\right)$ dada por $\varrho_{a}^{n}\left(x^{\prime}\right)=x_{\left.\right|_{U_{n}} ^{\prime}}^{\prime}(\cdot) a, \forall x^{\prime} \in E^{\prime}, \forall n \in \mathbb{N}$. É claro que, $\varrho_{a}^{n}\left(x^{\prime}\right) \in H_{b}\left(U_{n}, G\right)$, uma vez que $\sup _{x \in B}\left\|\varrho_{a}^{n}\left(x^{\prime}\right)(x)\right\| \leq\left\|x^{\prime}\right\| r$, onde $B \subset U_{n}$ é $U_{n}$-limitado e $\|x\| \leq r, \forall x \in B$.

Agora, da sentença (4.4) segue que

$$
\left\{\begin{array}{l}
A\left(\left[\varrho_{a}^{1}\left(x^{\prime}\right)\right]\right)=\left[\varrho_{a}\left(x^{\prime}\right) \circ \Phi_{1}\right], \forall x^{\prime} \in E^{\prime} \quad e \\
A\left(\left[\varrho_{a}^{n}\left(x^{\prime}\right)\right]\right)=\left[\varrho_{a}\left(x^{\prime}\right) \circ \Phi_{n}\right], \forall x^{\prime} \in E^{\prime}, \forall n \in \mathbb{N}
\end{array}\right.
$$

consequentemente, $\left[\varrho_{a}^{1}\left(x^{\prime}\right) \circ \Phi_{1}\right]=\left[\varrho_{a}^{n}\left(x^{\prime}\right) \circ \Phi_{n}\right], \forall x^{\prime} \in E^{\prime}, \forall n \in \mathbb{N}$. Ou seja $\varrho_{a}^{1}\left(x^{\prime}\right) \circ \Phi_{1} \sim \varrho_{a}^{n}\left(x^{\prime}\right) \circ \Phi_{n}, \forall x^{\prime} \in E^{\prime}, \forall n \in \mathbb{N}$. Assim, existe um conjunto aberto $W$ com $L \subset W \subset V_{m_{n}}$ tal que $\left(\varrho_{a}^{1}\left(x^{\prime}\right) \circ \Phi_{1}\right)_{\left.\right|_{W}}=\left(\varrho_{a}^{n}\left(x^{\prime}\right) \circ \Phi_{n}\right)_{\left.\right|_{W}}, \forall x^{\prime} \in E^{\prime}$.

Agora, seja $y \in V_{m_{n}}$, então $\exists z \in L$ tal que $y \in B_{\frac{1}{m_{n}}}(z)$. Como $L \subset W$ temos que $W \cap B_{\frac{1}{m_{n}}}(z) \neq \emptyset$ e consequentemente

$$
\left(\varrho_{a}^{1}\left(x^{\prime}\right) \circ \Phi_{1}\right)_{\left.\right|_{W \cap B} \frac{1}{m_{n}}(z)}=\left(\varrho_{a}^{n}\left(x^{\prime}\right) \circ \Phi_{n}\right)_{\left.\right|_{W \cap B} \frac{1}{m_{n}}(z)}, \forall x^{\prime} \in E^{\prime}
$$

Mas $B_{\frac{1}{m_{n}}}(z)$ é conexo $\operatorname{logo}\left(\varrho_{a}^{1}\left(x^{\prime}\right) \circ \Phi_{1}\right)_{\left.\right|_{{\frac{1}{m_{n}}}}(z)}=\left(\varrho_{a}^{n}\left(x^{\prime}\right) \circ \Phi_{n}\right)_{\left.\right|_{{\frac{1}{m_{n}}}}(z)}, \forall x^{\prime} \in E^{\prime}$. Assim, $\left(\varrho_{a}^{1}\left(x^{\prime}\right) \circ \Phi_{1}\right)(y)=\left(\varrho_{a}^{n}\left(x^{\prime}\right) \circ \Phi_{n}\right)(y), \forall y \in V_{m_{n}}, \forall x^{\prime} \in E^{\prime}$, então

$$
x^{\prime}\left(\Phi_{1}(y)\right)=x^{\prime}\left(\Phi_{n}(y)\right), \forall y \in V_{m_{n}}, \forall x^{\prime} \in E^{\prime}
$$


Usando o teorema de Hahn Banach, segue que

$$
\Phi(y)=\Phi_{1}(y)=\Phi_{n}(y), \forall y \in V_{m_{n}}
$$

Portanto, $\Phi_{\left.\right|_{\boldsymbol{V}_{m_{n}}}}=\Phi_{n}$ para cada $n \in \mathbb{N}$ e claramente $\Phi \in H_{b}\left(V_{m_{1}}, E\right)$.

Vamos agora mostrar que $\Phi(L) \subset K$. Da equação 4.6 temos que $\Phi(L) \subset U_{n}, \forall n \in \mathbb{N}$. Assim para cada $y \in L, \exists \xi_{n} \in K$ tal que $\left\|\Phi(y)-\xi_{n}\right\|<\frac{1}{n}$, para cada $n \in \mathbb{N}$, isto é, $d(\Phi(y), K)<\frac{1}{n}, \forall n \in \mathbb{N}, \forall y \in L$; fazendo $n \rightarrow \infty$, temos $d(\Phi(y), K)=0$, para cada $y \in L$ e consequentemente $\Phi(L) \subset K$. Assim, segue que $A([f])=[f \circ \Phi], \forall f \in$ $H_{b}\left(U_{n}, G\right), \forall n \in \mathbb{N}$, ou seja, $A=A_{\Phi}$.

Reciprocamente, suponhamos que $A$ é um operador de composição. Vamos provar que para cada aberto $U_{n} \supset K$ existem um aberto $V_{m_{n}} \supset L$ e um operador de composição $\widetilde{A}_{n}: H_{b}\left(U_{n}, G\right) \rightarrow H_{b}\left(V_{m_{n}}, G\right)$ tal que $\mathcal{J}_{m_{n}}^{L} \circ \widetilde{A}_{n}=A \circ \mathcal{J}_{n}^{K}$.

Seja $U_{n} \supset K$ um conjunto aberto. Segue da proposição 4.2 .2 que existem um conjunto aberto $V_{m_{l_{n}}} \supset L$ e um homomorfismo contínuo $\varphi_{n}: H_{b}\left(U_{n}, G\right) \rightarrow H_{b}\left(V_{m_{l_{n}}}, G\right)$ tal que $\mathcal{J}_{m_{l_{n}}}^{L} \circ \varphi_{n}=A \circ \mathcal{J}_{n}^{K}$

Dado que $A$ é um operador de composição, existem um conjunto aberto $V_{m_{0}} \supset L$, e $\Phi \in H_{b}\left(V_{m_{0}}, E\right)$ tal que

$$
A([f])=[f \circ \Phi], \forall f \in H_{b}\left(U_{n}, G\right) \text { e } \Phi(L) \subset K
$$

Seja $m_{k_{n}} \geq \max \left\{m_{l_{n}}, m_{0}\right\}$, então $V_{m_{k_{n}}} \subset V_{m_{l_{n}}} \cap V_{m_{0}}$ e a aplicação inclusão $H_{b}\left(V_{m_{l_{n}}}, G\right) \stackrel{i_{m_{l_{n}}}^{L}}{\longrightarrow} H_{b}\left(V_{m_{k_{n}}}, G\right)$ é tal que $\mathcal{J}_{m_{k_{n}}}^{L} \circ i_{m_{l_{n}}}^{L}=\mathcal{J}_{m_{l_{n}}}^{L}$. Considere o homomorfismo contínuo $\psi_{n}: H_{b}\left(U_{n}, G\right) \rightarrow H_{b}\left(V_{m_{k_{n}}}, G\right)$ dado por

$$
\psi_{n}(f)=i_{m_{l_{n}}}^{L} \circ \varphi_{n}(f), \forall f \in H_{b}\left(U_{n}, G\right)
$$

$\mathrm{e} \operatorname{assim} \mathcal{J}_{m_{k_{n}}}^{L} \circ \psi_{n}=A \circ \mathcal{J}_{n}^{K}$

De (4.7) e (4.8) temos que $[f \circ \Phi]=\left[\psi_{n}(f)\right], \forall f \in H_{b}\left(U_{n}, G\right)$ e portanto $f \circ \Phi \sim$ $\psi_{n}(f), \forall f \in H_{b}\left(U_{n}, G\right)$. Assim existe $V_{m_{n}} \subset V_{m_{k_{n}}}$ tal que $\psi_{n}(f)_{\left.\right|_{V_{m_{n}}}}=(f \circ \Phi)_{\left.\right|_{V_{m_{n}}}}$. Mas, $H_{b}\left(V_{m_{k_{n}}}, G\right) \stackrel{i_{m_{k_{n}}}^{L}}{\longrightarrow} H_{b}\left(V_{m_{n}}, G\right)$ e $\mathcal{J}_{m_{n}}^{L} \circ i_{m_{k_{n}}}^{L}=\mathcal{J}_{m_{k_{n}}}^{L}, \log \circ\left(i_{m_{k_{n}}}^{L} \circ \psi_{n}\right)(f)=(f \circ \Phi)_{\left.\right|_{V_{m_{n}}}}$. 
Agora, definimos $\widetilde{A}_{n}: H_{b}\left(U_{n}, G\right) \rightarrow H_{b}\left(V_{m_{n}}, G\right)$ por $\widetilde{A}_{n}=i_{m_{k_{n}}}^{L} \circ \psi_{n}$. Assim, $\widetilde{A}_{n}$ é um homomorfismo contínuo tal que $A \circ \mathcal{J}_{n}^{K}=\mathcal{J}_{m_{k_{n}}}^{L} \circ \psi_{n}=\mathcal{J}_{m_{n}}^{L} \circ i_{m_{k_{n}}}^{L} \circ \psi_{n}=\mathcal{J}_{m_{n}}^{L} \circ \widetilde{A}_{n} \mathrm{e}$ $\widetilde{A}_{n}(f)=(f \circ \Phi)_{V_{V_{m}}}$.

Assim, podemos definir uma aplicação $\Phi_{n}: V_{m_{n}} \rightarrow E$ por $\Phi_{n}=\Phi_{\left.\right|_{V_{m_{n}}}} \operatorname{com} \Phi_{n}\left(V_{m_{n}}\right) \subset$ $U_{n}$. Desta forma $\widetilde{A}_{n}(f)=f \circ \Phi_{n} \quad$ e $\quad \Phi_{n} \in H\left(V_{m_{n}}, E\right)$.

Vamos mostrar que $\Phi_{n} \in H_{b}\left(V_{m_{n}}, E\right)$. Seja $B \subset V_{m_{n}} \subset V_{m_{0}}$ um conjunto $V_{m_{n}{ }^{-}}$ limitado, pelo lema 3.1.2, temos que $B \subset V_{m_{0}}$ é $V_{m_{0}}$-limitado e como $\Phi \in H_{b}\left(V_{m_{0}}, G\right)$ temos que $\Phi(B) \subset E$ é limitado. Portanto $\Phi_{n}(B) \subset E$ é limitado, isto é, $\Phi_{n} \in H_{b}\left(V_{m_{n}}, E\right)$.

Assim, para cada aberto $U_{n} \supset K$ existem um conjunto aberto $V_{m_{n}} \supset L$ e um operador de composição $\widetilde{A}_{n}: H_{b}\left(U_{n}, G\right) \rightarrow H_{b}\left(V_{m_{n}}, G\right)$ tal que $A \circ \mathcal{J}_{n}^{K}=\mathcal{J}_{m_{n}}^{L} \circ \widetilde{A}_{n}$.

\subsection{Operadores de composição compactos entre álgebras de germes holomorfos a valores vetoriais}

Nesta seção estudamos os homomorfismos compactos entre álgebras de germes de funções holomorfas a valores em uma álgebra de Banach.

No teorema 4.3.2 damos uma condição necessária para homomorfismos contínuos compactos entre álgebras de germes de funções holomorfas a valores em uma álgebra de Banach. Na proposição 4.3.3 apresentamos uma condição necessária e suficiente para que operadores de composição entre álgebras de germes de funções holomorfas a valores em uma álgebra de Banach sejam compactos.

Na seguinte proposição mostramos que $\mathcal{H}(L, G)=\underset{m \in \mathbb{N}}{\lim _{m}} H_{b}\left(V_{m}, G\right)$ é limitadamente retroativo e será necessário na demonstração do teorema 4.3.2. A parte (b) da proposição será necessário na demonstração do teorema 4.4.1 que trata de operadores de composição fracamente compactos entre espaços de germes de funções holomorfas a valores vetoriais.

Proposição 4.3.1 Sejam E, $G$ espaços de Banach e $K \subset E$ um conjunto compacto. 


\section{Então,}

(a) O limite indutivo $\mathcal{H}(K, G)=\underset{n \in \mathbb{\mathbb { N }}}{\lim _{b}} H_{b}\left(U_{n}, G\right)$ é fortemente limitadamente retroativo.

(b) Para cada $n \in \mathbb{N}$, existe $m>n$ tal que

$$
\sigma\left(\mathcal{H}(K, G), \mathcal{H}(K, G)^{\prime}\right)_{\left.\right|_{B}}=\sigma\left(H_{b}\left(U_{m}, G\right), H_{b}\left(U_{m}, G\right)^{\prime}\right)_{\left.\right|_{B}}
$$

para cada conjunto limitado $B \subset H_{b}\left(U_{n}, G\right)$.

Demonstração. (a) Consideremos $\mathcal{H}(K, G)$ como o limite indutivo $\left(\mathcal{H}(K, G), \tau_{w}\right)=$ $\lim _{\substack{n \in \mathbb{N}\\}} H^{\infty}\left(U_{n}, G\right)$. Segue do teorema 21.21, pag. 358 de [4], que o limite indutivo $\left(\mathcal{H}(K, G), \tau_{w}\right)=\lim _{m \in \mathbb{N}} H^{\infty}\left(U_{n}, G\right)$ é limitadamente retroativo e pela observação $1.3 .2(3)$ temos que

$$
\left(\mathcal{H}(K, G), \tau_{w}\right)=\underset{n \in \mathbb{\mathbb { N }}}{\lim } H^{\infty}\left(U_{n}, G\right) \text { é fortemente limitadamente retroativo }
$$

Agora, mostraremos que o limite indutivo $\mathcal{H}(K, G)=\lim _{n \in \mathbb{\mathbb { N }}} H_{b}\left(U_{n}, G\right)$ verifica as seguintes condições do limite indutivo fortemente limitadamente retroativo.

(i) O limite indutivo $\mathcal{H}(K, G)=\underset{n \in \mathbb{\mathbb { N }}}{\lim _{b}} H_{b}\left(U_{n}, G\right)$ é regular.

Seja $\mathcal{X} \subset \mathcal{H}(K, G)$ um conjunto limitado, por 4.9 temos que $\left(\mathcal{H}(K, G), \tau_{w}\right)=$ $\lim _{n \in \mathbb{N}} H^{\infty}\left(U_{n}, G\right)$ é regular, logo existe $m \in \mathbb{N}$ tal que $\mathcal{X} \subset H^{\infty}\left(U_{m}, G\right)$ e é limitado. Como $H^{\infty}\left(U_{m}, G\right) \stackrel{i_{m}}{\longrightarrow} H_{b}\left(U_{m}, G\right)$, temos que $\mathcal{X} \subset H_{b}\left(U_{m}, G\right)$ e é limitado.

(ii) Sejam $m \in \mathbb{N}$ e $\mathcal{X} \subset H_{b}\left(U_{m}, G\right)$ um conjunto limitado. Então $\mathcal{I}_{m}^{K}(\mathcal{X}) \subset \mathcal{H}(K, G)$ é limitado e por 4.9 existe $n>m$ tal que $\mathcal{X} \subset H^{\infty}\left(U_{n}, G\right)$ e

$$
\left(\mathcal{H}(K, G), \tau_{w}\right)_{\left.\right|_{\mathcal{X}}}=H^{\infty}\left(U_{n}, G\right)_{\left.\right|_{\mathcal{X}}}
$$

Assim, temos as seguintes inclusões naturais no seguinte diagrama comutativo.

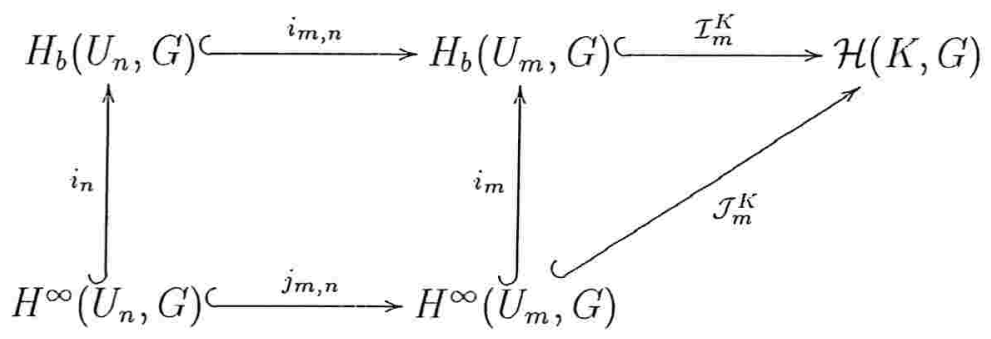


Da continuidade da inclusão $i_{n}$ temos que $\left(\mathcal{H}(K, G), \tau_{w}\right)_{\left.\right|_{\mathcal{X}}}=H_{b}\left(U_{n}, G\right)_{\left.\right|_{\mathcal{X}}}$.

Assim, o limite indutivo $\mathcal{H}(K, G)=\underset{n \in \mathbb{\mathbb { N }}}{\lim _{n}} H_{b}\left(U_{n}, G\right)$ é fortemente limitadamente retroativo. (b) O resultado segue de $(a)$ e da proposição 1.3 .4 .

Teorema 4.3.2 Sejam E, F espaços de Banach e $G$ uma álgebra de Banach. Sejam $K \subset E$ e $L \subset F$ subconjuntos compactos e $A:\left(\mathcal{H}(K, G), \tau_{w}\right) \rightarrow\left(\mathcal{H}(L, G), \tau_{w}\right)$ um homomorfismo contínuo compacto. Então para cada aberto $U_{n} \supset K$ existem um aberto $V_{m_{n}} \supset L$ e um homomorfismo contínuo compacto $\Psi_{n}: H_{b}\left(U_{n}, G\right) \rightarrow H_{b}\left(V_{m_{n}}, G\right)$ tal que $\mathcal{J}_{m_{n}}^{L} \circ \Psi_{n}=A \circ \mathcal{J}_{n}^{K}$.

Demonstração. Seja $U_{n} \subset E$ um conjunto aberto. Segue da proposição 4.2.2, que existem um aberto $V_{m_{r_{n}}} \supset L$ e um homomorfismo contínuo $\widetilde{A}_{n}: H_{b}\left(U_{n}, G\right) \rightarrow H_{b}\left(V_{m_{r_{n}}}, G\right)$ tal que $\mathcal{J}_{m_{r_{n}}}^{L} \circ \widetilde{A}_{n}=A \circ \mathcal{J}_{n}^{K}$.

Agora, para cada conjunto limitado $B$ de $H_{b}\left(V_{m_{r_{n}}}, G\right)$, pela proposição 4.3.1 (a), existe $m_{n}>m_{r_{n}}$ tal que

$$
\left(\mathcal{H}(L, G), \tau_{w}\right)_{\left.\right|_{B}}=H_{b}\left(V_{m_{n}}, G\right)_{\left.\right|_{B}}
$$

Como $H_{b}\left(V_{m_{r_{n}}}, G\right) \stackrel{i_{m_{r_{n}}}^{L}}{\longrightarrow} H_{b}\left(V_{m_{n}}, G\right) \quad$ e $\quad \mathcal{J}_{m_{n}}^{L} \circ i_{m_{r_{n}}}^{L}=\mathcal{J}_{m_{r_{n}}}^{L}$, podemos definir um homomorfismo contínuo $\Psi_{n}: H_{b}\left(U_{n}, G\right) \rightarrow H_{b}\left(V_{m_{n}}, G\right)$ por $\Psi_{n}=i_{m_{r_{n}}}^{L} \circ \widetilde{A}_{n}$ e assim $A \circ \mathcal{J}_{n}^{K}=\mathcal{J}_{m_{n}}^{L} \circ \Psi_{n}$. Como mostra o seguinte diagrama comutativo.

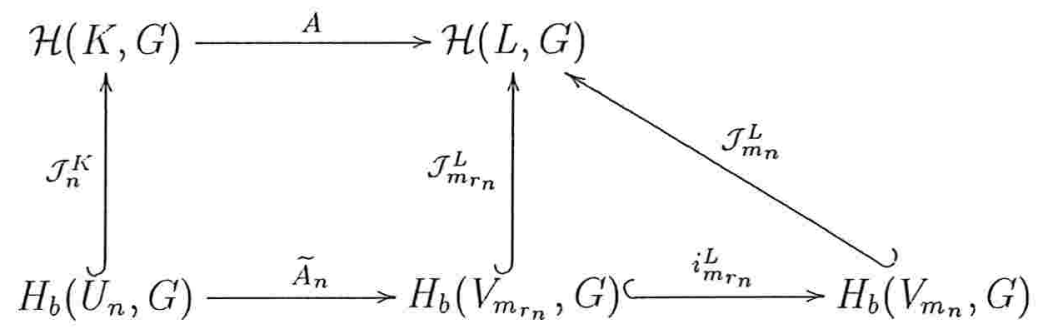

Afirmamos que o homomofismo $\Psi_{n}$ é compacto. De fato, seja $\mathcal{X} \subset H_{b}\left(U_{n}, G\right)$ limitado, $\operatorname{logo} \widetilde{A}_{n}(\mathcal{X}) \subset H_{b}\left(V_{m_{r_{n}}}, G\right)$ é limitado. Como $A$ é compacto, segue que $\mathcal{J}_{m_{r_{n}}}^{L}\left(\widetilde{A}_{n}(\mathcal{X})\right) \subset$ 
$\mathcal{H}(L, G)$ é relativamente compacto e como $\Psi_{n}(\mathcal{X})=i_{m_{r_{n}}}^{L}\left(\widetilde{A}_{n}(\mathcal{X})\right) \subset H_{b}\left(V_{m_{n}}, G\right)$ segue de (4.10) que é relativamente compacto. Logo $\Psi_{n}$ é compacto.

Assim, para cada aberto $U_{n} \supset K$ existem um aberto $V_{m_{n}} \supset L$ e um homomorfismo contínuo compacto $\Psi_{n}: H_{b}\left(U_{n}, G\right) \rightarrow H_{b}\left(V_{m_{n}}, G\right)$ tal que $\mathcal{J}_{m_{n}}^{L} \circ \Psi_{n}=A \circ \mathcal{J}_{n}^{K}$.

A seguir demonstraremos uma proposição que nos fornece uma condição necessária e suficiente para que operadores de composição sejam compactos.

Proposição 4.3.3 Sejam E, F espaços de Banach e G uma álgebra de Banach. Sejam $K \subset E$ e $L \subset F$ conjuntos compactos e $A:\left(\mathcal{H}(K, G), \tau_{w}\right) \rightarrow\left(\mathcal{H}(L, G), \tau_{w}\right)$ um operador de composição. Então A é compacto se, e somente se, para cada aberto $U_{n} \supset K$ existem um aberto $V_{m_{n}} \supset L$ e um operador de composição compacto $\Psi_{n}: H_{b}\left(U_{n}, G\right) \rightarrow H_{b}\left(V_{m_{n}}, G\right)$ tal que $\mathcal{J}_{m_{n}}^{L} \circ \Psi_{n}=A \circ \mathcal{J}_{n}^{K}$.

Demonstração. Vamos primeiramente supor que o operador de composição $A$ é compacto.

Segue do teorema 4.2 .3 que para cada aberto $U_{n} \supset K$ existem um aberto $V_{m_{r_{n}}} \supset L$ e um operador de composição $\widetilde{A}_{n}: H_{b}\left(U_{n}, G\right) \rightarrow H_{b}\left(V_{m_{r_{n}}}, G\right)$ tal que $\mathcal{J}_{m_{r_{n}}}^{L} \circ \psi_{n}=A \circ \mathcal{J}_{n}^{K}$. Agora, pela construção do teorema 4.3.2 temos que existem um conjunto aberto $V_{m_{n}} \supset L$ $\operatorname{com} H_{b}\left(V_{m_{r_{n}}}, G\right) \stackrel{i_{m_{r_{n}}}^{L}}{\longrightarrow} H_{b}\left(V_{m_{n}}, G\right)$ e um operador de composição compacto $\Psi_{n}=i_{m_{r_{n}}}^{L} \circ \widetilde{A}_{n}$ : $H_{b}\left(U_{n}, G\right) \rightarrow H_{b}\left(V_{m_{n}}, G\right)$ tal que $\mathcal{I}_{m_{n}}^{L} \circ \Psi_{n}=A \circ \mathcal{I}_{n}^{K}$.

Reciprocamente, suponhamos que para cada aberto $U_{n} \supset K$ existem um aberto $V_{m_{n}} \supset L$ e um operador de composição compacto $\Psi_{n}: H_{b}\left(U_{n}, G\right) \rightarrow H_{b}\left(V_{m_{n}}, G\right)$ tal que $\mathcal{I}_{m_{n}}^{L} \circ \Psi_{n}=A \circ \mathcal{I}_{n}^{K}$. Vamos provar que $A$ é compacto.

De fato, seja $\mathcal{X} \subset \mathcal{H}(K, G)$ um conjunto limitado. Vamos mostrar que $A(\mathcal{X}) \subset$ $\mathcal{H}(L, G)$ é relativamente compacto. Da proposição 4.3 .1 sabemos que existe $r \in \mathbb{N}$ tal que $\mathcal{X} \subset H_{b}\left(U_{r}, G\right)$ é limitado.

Por hipótese, existem um conjunto aberto $V_{m_{r}} \supset L$ e um operador de composição compacto $\Psi_{r}: H_{b}\left(U_{r}, G\right) \rightarrow H_{b}\left(V_{m_{r}}, G\right)$ tal que $\mathcal{J}_{m_{r}}^{L} \circ \Psi_{r}=A \circ \mathcal{J}_{r}^{K}$. Assim $\Psi_{r}(\mathcal{X}) \subset$ 
$H_{b}\left(V_{m_{r}}, G\right)$ é relativamente compacto, da continuidade de $\mathcal{J}_{m_{r}}^{L}$ concluimos que $A(\mathcal{X})=$ $\mathcal{J}_{m_{r}}^{L}\left(\Psi_{r}(\mathcal{X})\right) \subset \mathcal{H}(L, G)$ é relativamente compacto. Assim, $A$ é compacto.

\subsection{Operadores de composição fracamente compac- tos entre álgebras de germes holomorfos a valores vetoriais}

No teorema 4.4.1 mostramos uma condição necessária para homomorfismos contínuos fracamente compactos entre álgebras de germes de funções holomorfas a valores em uma álgebra de Banach. Agora na proposição 4.4 .2 mostramos uma condição necessária e suficiente para operadores de composição entre álgebras de germes de funções holomorfas a valores em uma álgebra de Banach sejam fracamente compactos.

Teorema 4.4.1 Sejam E, $F$ espaços de Banach e $G$ uma álgebra de Banach. Sejam $K \subset E$ e $L \subset F$ subconjuntos compactos e $A:\left(\mathcal{H}(K, G), \tau_{w}\right) \rightarrow\left(\mathcal{H}(L, G), \tau_{w}\right)$ um homomorfismo contínuo fracamente compacto. Então para cada aberto $U_{n} \supset K$, existem um aberto $V_{m_{n}} \supset L$ e um homomorfismo contínuo fracamente compacto $\Psi_{n}: H_{b}\left(U_{n}, G\right) \rightarrow$ $H_{b}\left(V_{m_{n}}, G\right)$ tal que $\mathcal{J}_{m_{n}}^{L} \circ \Psi_{n}=A \circ \mathcal{J}_{n}^{K}$.

Demonstração. Seja $U_{n} \subset E$ um conjunto aberto, pela proposição 4.2.2, existem $V_{m_{r_{n}}} \supset L$ aberto e $\widetilde{A}_{n}: H_{b}\left(U_{n}, G\right) \rightarrow H_{b}\left(V_{m_{r_{n}}}, G\right)$ um homomorfismo contínuo tal que $\mathcal{J}_{m_{r_{n}}}^{L} \circ \widetilde{A}_{n}=A \circ \mathcal{J}_{n}^{K}$.

Agora, pela proposição $4.3 .1(b)$, existe $m_{n}>m_{r_{n}}$ tal que

$$
\left.\sigma\left(\mathcal{H}(L, G), \mathcal{H}(L, G)^{\prime}\right)\right|_{\left.\right|_{B}}=\sigma\left(H_{b}\left(V_{m_{n}}, G\right), H_{b}\left(V_{m_{n}}, G\right)^{\prime}\right)_{\left.\right|_{B}}
$$

para cada conjunto limitado $B$ de $H_{b}\left(V_{m_{r_{n}}}, G\right)$. Como $H_{b}\left(V_{m_{r_{n}}}, G\right) \stackrel{i_{m_{r_{n}}}^{L}}{\hookrightarrow} H_{b}\left(V_{m_{n}}, G\right)$ e $\mathcal{J}_{m_{n}}^{L} \circ i_{m_{r_{n}}}^{L}=\mathcal{J}_{m_{r_{n}}}^{L}$, podemos definir um homomorfismo contínuo

$\Psi_{n}: H_{b}\left(U_{n}, G\right) \rightarrow H_{b}\left(V_{m_{n}}, G\right)$ por $\Psi_{n}=i_{m_{r_{n}}}^{L} \circ \widetilde{A}_{n}$ e $\operatorname{assim} A \circ \mathcal{J}_{n}^{K}=\mathcal{J}_{m_{n}}^{L} \circ \Psi_{n}$. Como mostra o seguinte diagrama comutativo. 


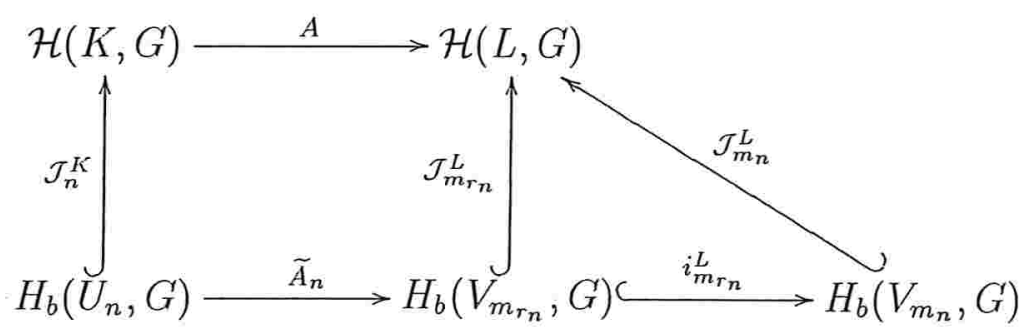

Afirmamos que o homomorfismo contínuo $\Psi_{n}$ é fracamente compacto. De fato, seja $\mathcal{X} \subset H_{b}\left(U_{n}, G\right)$ limitado, logo $\widetilde{A}_{n}(\mathcal{X}) \subset H_{b}\left(V_{m_{r_{n}}}, G\right)$ é limitado. Como $A$ é fracamente compacto, segue que $\mathcal{J}_{m_{r_{n}}}^{L}\left(\widetilde{A}_{n}(\mathcal{X})\right) \subset \mathcal{H}(L, G)$ é relativamente fracamente compacto e como $\Psi_{n}(\mathcal{X})=i_{m_{r_{n}}}^{L}\left(\widetilde{A}_{n}(\mathcal{X})\right) \subset H_{b}\left(V_{m_{n}}, G\right)$ segue de (4.11) que é relativamente fracamente compacto. Logo $\Psi_{n}$ é fracamente compacto.

Assim, para cada aberto $U_{n} \supset K$ existem um aberto $V_{m_{n}} \supset L$ e um homomorfismo fracamente compacto $\Psi_{n}: H_{b}\left(U_{n}, G\right) \rightarrow H_{b}\left(V_{m_{n}}, G\right)$ tal que $\mathcal{J}_{m_{n}}^{L} \circ \Psi_{n}=A \circ \mathcal{J}_{n}^{K}$.

A seguir demonstramos uma proposição que nos fornece uma condição necessária e suficiente para que operadores de composição entre álgebras de germes de funções holomorfas a valores em uma álgebra de Banach sejam fracamente compactos.

Proposição 4.4.2 Sejam E, F espaços de Banach e $G$ uma álgebra de Banach. Sejam $K \subset E$ e $L \subset F$ subconjuntos compactos e $A:\left(\mathcal{H}(K, G), \tau_{w}\right) \rightarrow\left(\mathcal{H}(L, G), \tau_{w}\right)$ um operador de composição. Então A é fracamente compacto se, e somente se, para cada aberto $U_{n} \supset K$ existem um aberto $V_{m_{n}} \supset L$ e um operador de composição fracamente compacto

$\Psi_{n}: H_{b}\left(U_{n}, G\right) \rightarrow H_{b}\left(V_{m_{n}}, G\right)$ tal que $\mathcal{J}_{m_{n}}^{L} \circ \Psi_{n}=A \circ \mathcal{J}_{n}^{K}$.

Demonstração. Vamos primeiramente supor que $A$ é um operador de composição fracamente compacto.

Segue do teorema 4.2.3, que para cada aberto $U_{n} \supset K$ existem um aberto $V_{m_{r_{n}}} \supset L$ e um operador de composição $\widetilde{A}_{n}: H_{b}\left(U_{n}, G\right) \rightarrow H_{b}\left(V_{m_{r_{n}}}, G\right)$ tal que $\mathcal{J}_{m_{r_{n}}}^{L} \circ \widetilde{A}_{n}=A \circ \mathcal{J}_{n}^{K}$. Agora, pela construção do teorema 4.4.1 temos que existem um 
conjunto aberto $V_{m_{n}} \supset L \operatorname{com} H_{b}\left(V_{m_{r_{n}}}, G\right) \stackrel{i_{m_{r_{n}}}^{L}}{\longrightarrow} H_{b}\left(V_{m_{n}}, G\right)$ e um operador de composição fracamente compacto $\Psi_{n}=i_{m_{r_{n}}}^{L} \circ \widetilde{A}_{n}: H_{b}\left(U_{n}, G\right) \rightarrow H_{b}\left(V_{m_{n}}, G\right)$ tal que $\mathcal{J}_{m_{n}}^{L} \circ \Psi_{n}=A \circ \mathcal{J}_{n}^{K}$ (ver o diagrama 4.12).

Reciprocamente, seja $\mathcal{X} \subset \mathcal{H}(K, G)$ um conjunto limitado. Segue da proposição 4.3.1 que existe $r \in \mathbb{N}$ tal que $\mathcal{X} \subset H_{b}\left(U_{r}, G\right)$ é limitado. Assim, por hipótese, existem um conjunto aberto $V_{m_{r}} \supset L$ e um operador de composição fracamente compacto $\Psi_{r}: H_{b}\left(U_{r}, G\right) \rightarrow H_{b}\left(V_{m_{r}}, G\right)$ tal que $\mathcal{J}_{m_{r}}^{L} \circ \Psi_{r}=A \circ \mathcal{J}_{r}^{K}$. Como $\Psi_{r}(\mathcal{X}) \subset H_{b}\left(V_{m_{r}}, G\right)$ é relativamente fracamente compacto e $A(\mathcal{X})=\mathcal{J}_{m_{r}}^{L}\left(\Psi_{r}(\mathcal{X})\right) \subset \mathcal{H}(L, G)$, segue da continuidade de $\mathcal{J}_{m_{r}}^{L}$ que $A(\mathcal{X})$ é é relativamente fracamente compacto.

Portanto, $A$ é fracamente compacto. 


\section{Referências Bibliográficas}

[1] R. Alencar, R. Aron and S. Dineen: A reflexive space of holomorphic functions in infinitely many variables, Proc. Amer. Math. Soc. 90 (1984), 407-411.

[2] J. M. Ansemil and S. Ponte An example of a quasi-normable Fréchet function space which is not a Schwartz space, Functional analysis, holomorphy, and approximation theory (Proc. Sem., Univ. Fed. Rio de Janeiro, Rio de Janeiro, 1978), pg. 1-8, Lect. Notes in Math. 843, Springer, Berlin, 1981.

[3] K. D. Bierstedt: An introduction to locally convex inductive limits, Funct. Anal. \& Appl., World Sci. Publ., Singapore, (1988), 35-133.

[4] S. B. Chae: Holomorphy and Calculus in Normed Spaces, Marcel Dekker, 1985.

[5] J. Diestel: A survey of results to the Dunford-Pettis property, Proc. Conference on Integration, Topology, and Geometry in Linear Spaces(Univ. North Carolina, Chapel Hill, N. C., 1979)-Contemp. Math., 2, AMS, Providence, R. I., (1980), 15-60.

[6] J. Diestel: Sequences and Series in Banach Spaces, Graduate texts in Math., $N^{\circ}$ 92, Springer, 1984.

[7] S. Dineen: Complex Analysis in Locally Convex Spaces, Math. Stud. 57, North-Holland, 1981.

[8] S. Dineen: Complex Analysis on Infinite Dimensional Sapaces, Springer-Verlag London Berlin Heidelberg, 1999.

[9] K. Floret: Weakly Compact Sets, Lect. Notes Math., 801 (1978). 
[10] R. E. Edwards: Functional Analysis, Theory and Applications, Holt Rinehart and Winston, New York, 1965.

[11] M. González, J. Gutiérrez: Schauder type theorems for differentiable and holomorphic mappings, Math. Nachr. 122 (1996), 325-343.

[12] M. González, J. Gutiérrez: Gantmacher type theorems for holomorphic mappings, Math. Nachr. 186 (1997), 131-145.

[13] P. Galindo, M. L. Lourenço and L. A. Moraes: Compact and weakly compact homomorphisms on Fréchet algebras of holomorphic functions, Math. Nach. A aparecer...

[14] A. Grothendieck: Produits Tensoriels Topologiques et Espaces Nucléaires, Memoirs Am. Math. Soc. 16 (1955).

[15] J. Horvath: Topological Vector Spaces and Distributions, Vol. 1. Addison-Wesley, Massachusetts, 1966.

[16] J. M. Isidro: Characterization of the spectrum of some topological algebras of holomorphic functions, Advances in Holomorphy, J.A. Barroso (ed.). North-Holland Publishing Company, (1979), 407-416.

[17] J. M. Isidro: Topological duality on the functional space $\left(H_{b}(U, F), \tau_{b}\right)$, Proc. Royal Irish Acad. 79A (1979), 115-130.

[18] J. Mujica: Complex Analysis in Banach Spaces, Math. Stud. 120, North-Holland, 1986.

[19] J. Mujica: Gérmenes Holomorfos y Funciones Holomorfas en Espacios de Fréchet, Publicaciones del departamento de teoria de funciones. Universidad de Santiago de Compostela, $N^{\text {ro }} 1,1978$.

[20] J. Mujica: Spaces of germs of holomorphic functions, Adv. Math. Suppl. Stud., 4, Academic Press, (1979), 1-41.

[21] J. Mujica: Ideals of holomorphic functions on Fréchet spaces, Advances in Holomorphy, Ed. J. A. Barroso, North-Holland Publishing Company, 1979.

[22] J. Mujica: The Oka-Weil theorem in locally convex spaces with the approximation property, Séminaire Paul Krée 1977/1978. Institute Henri Poincaré, Paris, 1979. 
[23] J. Mujica: Ideals of holomorphic functions on Tsirelson's space, Arch. Math. 76 (2001), 292-298.

[24] L. Nachbin: Lectures on the Theory of Distributions, University of Rochester (1963). Reproduced in textos de Matemática, 15, Universidade Federal de Pernambuco, Recife, Brazil (1964).

[25] L. Nachbin: On spaces of holomorphic functions of a given type, Funct. Anal., Irvine, 1966, Ed. B. R. Gelbaum, Academic Press, (1967), 50-70.

[26] L. Nachbin: Topology on spaces of holomorphic mappings, Ergenisse der Mathematik und ihrer Grenzgebiete 47, Springer-Verlag, 1969.

[27] L. Nachbin: Topics on Topological Vector Spaces, Textos de Métodos Matemáticos Da Universidade Federal Do Rio De Janeiro, (1994).

[28] O. Nicodemi: Homomorphisms of algebras of germs of holomorphic functions, Functional Analysis, Holomorphy and Approximation, Theory, Ed. S. Machado, Lect. Notes in Math. 843 (1981), 534-546.

[29] A. Pelczynski: On weakly compact polynomial operators on B-spaces with Dunford-Pettis property, Bull. de L'Acad. Polon. des Sciences, Sér. Sci. Math., 11 (1963), 371-378.

[30] R. A. Ryan: Weakly compact holomorphic mappings on Banach spaces, Pacific J. Math. 131 (1988), 179-190.

[31] J. O. Stevenson: Holomorphy of composition, Infinite Dimensional holomorphy and Aplications, M. C. Matos (ed.), North-Holland, 12 (1977), 397-424.

[32] B. Tsirelson: Not every Banach space contains an imbedding of $\ell_{p}$ ou $\mathrm{c}_{0}$, Funct. Anal. Appl. 8 (1974), 138-144.

[33] M. De Wilde: Critères de densité et de séparation dans des limites projectives et inductives dénombrables, Bull. de la Soc. Roy. des Sc. de Liège, 41, $N^{o} 3-4$, (1972), 155-162. 